\title{
Multi-trace correlators from permutations as moduli space
}

\author{
Ryo Suzuki \\ School of Physics, Korea Institute for Advanced Study, \\ 85 Hoegiro, Dongdaemun-Gu, Seoul 02455, Korea \\ E-mail: rsuzuki.mp@gmail.com
}

AbStRact: We study the $n$-point functions of scalar multi-trace operators in the $\mathrm{U}\left(N_{c}\right)$ gauge theory with adjacent scalars, such as $\mathcal{N}=4$ super Yang-Mills, at tree-level by using finite group methods. We derive a set of formulae of the general $n$-point functions, valid for general $n$ and to all orders of $1 / N_{c}$. In one formula, the sum over Feynman graphs becomes a topological partition function on $\Sigma_{0, n}$ with a discrete gauge group, which resembles closed string interactions. In another formula, a new skeleton reduction of Feynman graphs generates connected ribbon graphs, which resembles open string interaction. We define the moduli space $\mathcal{M}_{g, n}^{\text {gauge }}$ from the space of skeleton-reduced graphs in the connected $n$-point function of gauge theory. This moduli space is a proper subset of $\mathcal{M}_{g, n}$ stratified by the genus, and its top component gives a simple triangulation of $\Sigma_{g, n}$.

KEYwords: 1/N Expansion, AdS-CFT Correspondence, Matrix Models

ARXIV EPRINT: 1810.09478 


\section{Contents}

1 Introduction 1

1.1 Outline of ideas 3

1.2 Why many formulae? 5

2 Correlators from permutations $\quad 6$

$\begin{array}{lll}2.1 & S_{2 L} \text { (or vertex-based) formula } & 6\end{array}$

$2.2 S_{L}$ (or edge-based) formula 9

2.2.1 Symmetry of the $S_{L}$ formula 11

$\begin{array}{ll}2.2 .2 \text { Cayley graph } & 11\end{array}$

$\begin{array}{lll}2.2 .3 & \text { Extremal case } & 13\end{array}$

$\begin{array}{lll}2.3 & \text { Face-based formulae } & 14\end{array}$

3 Pants decomposition $\quad 18$

4 Skeleton reduction $\quad 21$

4.1 Open two-point functions 22

4.2 Relabeling Wick-contractions 24

$\begin{array}{lll}4.3 & \text { Face-based skeleton formula } & 26\end{array}$

$\begin{array}{lll}\text { 4.3.1 Sum over the skeleton faces } & 26\end{array}$

$\begin{array}{lll}\text { 4.3.2 } & \text { Reconstruction of correlators } & 28\end{array}$

$\begin{array}{lll}\text { 4.3.3 Sum over graphs } & 30\end{array}$

5 Geometry from permutations $\quad 32$

5.1 Ribbon graph and Riemann surface 32

5.2 Constraints from gauge theory 33

5.3 Examples 35

6 Metric ribbon graphs in string theory 41

$\begin{array}{lll}7 & \text { Conclusion and outlook } & 42\end{array}$

A Notation $\quad 43$

A.1 Permutations 43

A.2 Permutation basis of operators $\quad 45$

A.3 Feynman diagrams in the double-line notation 46

A.4 Summary of skeleton reduction $\quad 47$

B Another face-based formula $\quad 49$ 
C Vertex-based skeleton formula $\quad \mathbf{5 0}$

C.1 Gluing open two-point 50

$\begin{array}{lll}\text { C.2 Face permutations of the skeleton graph } & 52\end{array}$

C.3 Space of the reduced Wick-contractions 52

C.3.1 Partitions of an operator 53

C.3.2 Space of internal Wick-contractions 54

C.3.3 Space of external Wick-contractions 55

C.3.4 Rewriting the $n$-point functions 56

D More on geometry and graphs $\quad 56$

D.1 Moduli space $\quad 56$

$\begin{array}{ll}\text { D.2 Ribbon graphs } & 57\end{array}$

D.3 Quadratic differentials $\quad 58$

D.4 Drawing bipartite ribbon graphs 60

D.5 Feynman graphs on a Riemann surface $\quad 62$

D.6 Counting the dimensions of $\mathcal{M}_{g, n}^{\text {gauge }} 63$

E Details of the skeleton reduction $\quad 64$

\section{Introduction}

In the large $N_{c}$ gauge theories, Riemann surfaces are carved out by the Feynman diagrams in the double-line notation [1]. This observation was a precursor of the AdS/CFT correspondence, that is the conjectured duality between gauge and string theories [2]. The AdS/CFT correspondence can be checked explicitly at any values of the 't Hooft coupling $\lambda=N_{c} g_{\mathrm{YM}}^{2}$ in the integrable setup, such as $\mathcal{N}=4$ super Yang-Mills (SYM) in four dimensions and superstring on $\mathrm{AdS}_{5} \times \mathrm{S}^{5}[3,4]$.

The AdS/CFT correspondence beyond the large $N_{c}$ limit is not well-understood, including the question of integrability. It is known that the non-planar integrability, even if exists, does not protect the spectrum, because the $1 / N_{c}$ corrections lift the spectral degeneracy of the planar $\mathcal{N}=4 \mathrm{SYM}$ [5]. One of the promising ideas in this direction is based on the hexagon form factor [6], which can capture $1 / N_{c}$ corrections [7-9].

Integrability predictions are often formulated and refined with the help of extensive perturbative data. At weak coupling, the $1 / N_{c}$ corrections can be computed by summing over the graphs at a fixed genus [10]. However, this is tedious, and the finite group method is more efficient. There one expresses multi-trace operators in terms of permutations, and applies finite-group Fourier-transform to obtain a new basis of gauge-invariant operators labeled by representations or Young tableaux [11]. The representation basis diagonalizes tree-level two-point functions at any $N_{c}$, and solve finite $N_{c}$ constraints $[12,13]$. The finite group methods are used to compute various quantities, such as partition functions, two-point functions, and recently extremal $n$-point functions [14]. 
We ask two questions in this paper. How do we find general non-extremal $n$-point functions in the finite group methods, at any $n$ and to any orders of $1 / N_{c}$ ? And how do these correlators describe Riemann surfaces?

A similar problem was studied in the Hermitian matrix model, which is a simpler version of large $N_{c}$ gauge theory [15]. This matrix model describes two-dimensional gravity in the continuum limit, and its exact free energy is given by the $\tau$-function of the $\mathrm{KdV}$ hierarchy $[16,17]$.

We will study the tree-level $n$-point functions of scalar operators in $\mathcal{N}=4 \mathrm{SYM}$ with $\mathrm{U}\left(N_{c}\right)$ gauge group. We express Feynman graphs in terms of permutations, and describe the space of Wick-contractions in an algebraic manner. We obtain a formula which naturally factorizes into the product of pairs of pants, i.e. three-point functions. This formula is invariant different pants decomposition, and resembles the interaction of $n$ closed strings.

Then we perform a skeleton reduction to the Feynman graphs in the $n$-point functions. Under the skeleton reduction, Feynman graphs become connected metric ribbon graphs. It is known that there is an isomorphism between the space of connected metric ribbon graphs and the decorated moduli space of Riemann surfaces [18-21]. Thus we define the moduli space of Riemann surfaces in gauge theory $\mathcal{M}_{g, n}^{\text {gauge }}$ as the space of Wick-contractions in the skeleton-reduced Feynman graphs. These graphs resemble the interaction of open strings which triangulates $\Sigma_{g, n}$.

The gauge theory moduli space $\mathcal{M}_{g, n}^{\text {gauge }}$ exhibits two properties. First, it is a proper subset of the moduli space of the decorated arithmetic Riemann surfaces, equivalent to the connected integral ribbon graphs [22, 23]. Second, our definition of the skeleton reduction stratifies $\mathcal{M}_{g, n}^{\text {gauge }}$ by genus, meaning that the diagrams with smaller genera contribute to higher powers of $1 / N_{c}{ }^{1}$

In [27] the correlators of gauge theory are used to define an effective two-dimensional worldsheet theory, by rewriting propagators in the Schwinger parameterization. This "CFT" has been studied in detail, and its unusual properties have been found [28-34], including $\mathcal{M}_{g, n}^{\text {gauge }} \subsetneq \mathcal{M}_{g, n}$ mentioned above. ${ }^{2}$ Another idea is to interpret the correlators of Hermitian matrix model as the counting of holomorphic maps [36]. It is argued that the Gaussian matrix model is dual to the A-model topological string on $\mathbb{P}^{1}$ [37-39], similar to the Eguchi-Yang model [40].

Our results may be regarded as a variation of the open/closed duality, exploring the $n$-point functions of a gauge theory at tree-level from various aspects. Correspondingly, this paper consists of two parts. In sections 2 and 3, closed-string-like formulae of the $n$ point functions are discussed, where one of the formulae has natural pants decomposition. In sections 4 and 5, open-string-like formulae of the $n$-point functions are discussed, where the skeleton-reduced graphs define the moduli space $\mathcal{M}_{g, n}^{\text {gauge }}$ through triangulation.

\footnotetext{
${ }^{1}$ This is different from the stratification arising in different situations, such as the Deligne-Mumford compactification of the moduli space [24] (see also [16, 25]), hermitian matrix model [26] and hexagonization program $[8,9]$.

${ }^{2}$ Matrix models cover the full moduli space, and describe CFT's in the continuum limit [35].
} 


\subsection{Outline of ideas}

Our objectives in this paper are to compute $n$-point functions by using permutations, and to define $\mathcal{M}_{g, n}^{\text {gauge }}$ by using permutations.

Let $\mathcal{O}_{i}$ be a general multi-trace scalar operator of length $L_{i}$ in $\mathcal{N}=4 \mathrm{SYM},{ }^{3}$ whose color structure is specified by the permutation $\alpha_{i} \in S_{L_{i}}$ and flavor structure unspecified. The tree-level $n$-point function is schematically written as the sum over all possible sets of Wick-contractions,

$$
G_{n} \equiv\left\langle\mathcal{O}_{1} \mathcal{O}_{2} \ldots \mathcal{O}_{n}\right\rangle=\sum_{\text {Wick } \in \mathcal{W}} \prod_{i<j}^{n} \operatorname{Wick}\left(\mathcal{O}_{i}, \mathcal{O}_{j}\right)
$$

The space of Wick-contractions $\mathcal{W}$ decomposes as

$$
\mathcal{W}=\bigsqcup_{\left\{\ell_{i j}\right\}} \mathcal{W}\left(\left\{\ell_{i j}\right\}\right)
$$

where $\ell_{i j}$ is the number of all Wick-contractions between $\mathcal{O}_{i}$ and $\mathcal{O}_{j}$, also called bridge length. The bridge lengths satisfy

$$
\ell_{i i}=0, \quad \ell_{i j}=\ell_{j i}, \quad L_{i}=\sum_{j \neq i} \ell_{i j}, \quad L \equiv \sum_{1 \leq i<j \leq n} l_{i j}=\frac{1}{2} \sum_{i=1}^{n} L_{i} .
$$

The space of Wick-contractions is equivalent to the sum over Feynman diagrams. In order to study graphs, a permutation is a powerful tool. Feynman diagram can be interpreted as the Cayley graph generated by a finite group. The graph data (vertices, edges and faces) are translated into a triple of permutations. Hence, there should be permutation-based formulae of $G_{n}$. Once we start looking for such formulae, we encounter the following questions.

i) How to multiply the elements of different permutation groups?

ii) How to sum over $\left\{\ell_{i j}\right\}$ ?

Our answer to both is by embedding,

i) to embed $\left\{\alpha_{i}\right\}$ into a big permutation group to make the group multiplication welldefined.

ii) to embed $\mathcal{W}$ into a larger but simpler space by adding unphysical Wick-contractions. These tricks simplify (1.1) enormously.

We will obtain three different expressions of $G_{n}$ in section 2 , which we call vertexbased, edge-based and face-based formulae. Let us explain the edge-based formula, which reads

$$
\begin{aligned}
& G_{n}=\frac{1}{\prod_{i=1}^{n}\left(L-L_{i}\right) !} \frac{1}{L !} \sum_{\left\{U_{i}\right\} \in S_{L}^{\otimes n}}\left(\prod_{p=1}^{L} h^{\hat{A}_{U_{1}(p)}^{(1)} \hat{A}_{U_{2}(p)}^{(2)} \cdots \hat{A}_{U_{n}(p)}^{(n)}}\right)
\end{aligned}
$$

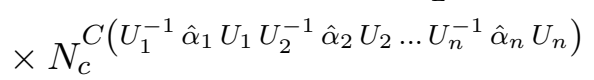

\footnotetext{
${ }^{3}$ More generally multi-trace operators which consist of scalars in the adjoint representation of $\mathrm{U}\left(N_{c}\right)$.
} 
where $C(\sigma)$ counts the number of cycles in $\sigma, \hat{\alpha}_{i}$ specifies the color structure of the $i$-th multi-trace operator, and $h^{A_{1} A_{2} \ldots A_{n}}$ is related to the flavor inner-product. This formula follows from the idea of extending the operators by adding identity fields,

$$
\mathcal{O}_{i} \sim\left(\Phi^{\hat{A}_{1}^{(i)}}\right)_{a_{\hat{\alpha}_{i}(1)}}^{a_{1}}\left(\Phi^{\hat{A}_{2}^{(i)}}\right)_{a_{\hat{\alpha}_{i}(2)}}^{a_{2}} \ldots\left(\Phi^{\hat{A}_{L}^{(i)}}\right)_{a_{\hat{\alpha}_{i}(L)}}^{a_{L}}, \quad\left(\hat{\alpha}_{i} \in S_{L_{i}} \times \mathbf{1}^{L-L_{i}} \subset S_{L}\right)
$$

where $\Phi^{\hat{A}_{p}^{(i)}}=\mathbf{1}$ for $L_{i}<p \leq L$.

We decompose the formula (2.28) into the product of pairs of pants in section 3

$$
G_{n} \sim \operatorname{Glue}\left(\mathcal{X}\left[\mathcal{S}_{1}\right], \mathcal{X}\left[\mathcal{S}_{2}\right], \ldots, \mathcal{X}\left[\mathcal{S}_{n-2}\right]\right), \quad \Sigma_{0, n}=\mathcal{S}_{1} \amalg \mathcal{S}_{2} \amalg \cdots \amalg \mathcal{S}_{n-2}
$$

where $\left\{\mathcal{S}_{f}\right\}$ is a local three-point interaction. The r.h.s. of (3.1) depends only on the topology of $\Sigma_{0, n}$. We introduce a defect on $\Sigma_{0, n}$ which carries all information on the powers of $N_{c}$. As a result, no higher-genus surfaces show up.

In section 4 , we study $G_{n}$ in the opposite way by reduction, and take the sum over $\left\{\ell_{i j}\right\}$ literally. This sum is related to the moduli space of Riemann surfaces, which becomes transparent after a skeleton reduction. The skeleton reduction can be defined by taking the Wick-contractions in two steps. Let us define a partition of $L_{i}$ by $\ell_{i} \equiv\left(\ell_{i \mid 1}, \ell_{i \mid 2}, \ldots, \ell_{i \mid \bar{L}_{i}}\right) \vdash$ $L_{i}$. We denote a sequence of consecutive $\boldsymbol{\ell}_{i \mid r}$ fields in $\mathcal{O}_{i}$ by $\boldsymbol{\Phi}_{r}^{(i)}$, and define the reduced single-trace operator by

$$
\overline{\mathcal{O}}_{i}=\operatorname{tr}\left(\boldsymbol{\Phi}_{1}^{(i)} \boldsymbol{\Phi}_{2}^{(i)} \ldots \boldsymbol{\Phi}_{\bar{L}_{i}}^{(i)}\right) .
$$

There are many ways to create $\overline{\mathcal{O}}_{i}$ from $\mathcal{O}_{i}$, which we call partitions of $\mathcal{O}_{i}$. We define the space of the external Wick-contractions by all possible pairings of $\{(i, r),(j, s)\}$ in $\boldsymbol{\Phi}_{r}^{(i)}$ and $\boldsymbol{\Phi}_{s}^{(j)}$. Then we impose the internal Wick-contraction rule between $\boldsymbol{\Phi}_{r}^{(i)}$ and $\boldsymbol{\Phi}_{s}^{(j)}$, which is non-zero only if $\boldsymbol{\ell}_{i \mid r}=\boldsymbol{\ell}_{j \mid s}$ and expressed as a sum over the internal pairing map $\tau \in S_{\boldsymbol{\ell}_{i \mid r}}$. Since $\tau$ contains non-planar Wick-contractions, the topology of the skeleton-reduced graphs is greatly simplified.

We will obtain two more permutation-based formulae for $G_{n}$, which we call vertexbased and face-based skeleton formulae. The face-based skeleton formula for the $n$-point function reads

$$
\left.G_{n} \sim \sum_{\ell \vdash L} \frac{1}{|\operatorname{Aut} V(\boldsymbol{\ell})|} \sum_{\nu \in\left(S_{2 \bar{L}}^{\times \times}\right)_{\text {phys }}} \sum_{\boldsymbol{\tau} \in S_{\boldsymbol{\tau}}} \mathbb{F}(\boldsymbol{\tau} \mid \nu)\right|_{\text {constraints }}
$$

where $\boldsymbol{\ell}=\left(\ell_{1}, \ell_{2}, \ldots, \ell_{\bar{L}}\right) \vdash L$, and $S_{2 \bar{L}}^{\times \times}$consists of the elements of $S_{2 \bar{L}}$ without one- or two-cycles. The permutation $\nu \in S_{2 \bar{L}}^{\times \times}$defines a skeleton graph. The function $\mathbb{F}$ maps a set of skeleton graphs to a polynomial of color and flavor factors, and takes a complicated form

$$
\mathbb{F}(\boldsymbol{\tau} \mid \nu)=N_{c}^{\bar{Z}(\boldsymbol{\tau})+C(\bar{\omega})} \sum_{\bar{\beta} \in \mathbf{C R}\left(\bar{\alpha}_{\bullet}\right)} \sum_{\boldsymbol{z} \in \mathbb{Z}_{\alpha}} \prod_{s=1}^{\bar{L}} \boldsymbol{z} \cdot h\left(\boldsymbol{\tau} \mid \bar{\mu}_{1, s} \bar{\mu}_{1, s^{\prime}} \ldots \bar{\mu}_{n, s} \bar{\mu}_{n, s^{\prime}}\right)
$$

where $\bar{\omega}$ is related to $\nu$. The set $\left(S_{2 \bar{L}}^{\times \times}\right)_{\text {phys }}$ is defined so that it corresponds to a skeleton graph

$$
\{\text { Skeleton graphs with } 2 \bar{L} \text { unlabeled vertices }\} \quad \leftrightarrow \quad \nu \in\left(S_{2 \bar{L}}^{\times \times} / \operatorname{Aut} V\right)_{\text {phys }} .
$$


From this correspondence, we translate the formula (4.50) into a sum over graphs as

$$
\left(G_{n}\right)_{\text {connected }}=\sum_{g \geq 0} \sum_{\Gamma(\ell) \in \mathcal{M}_{g, n}^{\text {gauge }}\left(\left\{L_{i}\right\}\right)} \frac{1}{|\operatorname{Aut} V(\ell)|} \sum_{\boldsymbol{\tau} \in S_{\boldsymbol{\tau}}} \mathscr{F}(\boldsymbol{\tau} \mid \Gamma)
$$

where $\mathscr{F}(\boldsymbol{\tau} \mid \Gamma)$ is rewriting of $\mathbb{F}(\boldsymbol{\tau} \mid \nu)$ in the language of graphs.

We call $\mathcal{M}_{g, n}^{\text {gauge }}\left(\left\{L_{i}\right\}\right)$ the moduli space of gauge theory. This is a proper subset of the connected metric ribbon graphs, or equivalently of the decorated moduli space of Riemann surfaces.

$$
\begin{aligned}
& \mathcal{M}_{g, n}^{\text {gauge }}\left(\left\{L_{i}\right\}\right) \subsetneq \mathcal{M}_{g, n} \equiv\left(\text { Choice of }\left\{\ell_{E}>0\right\}\right) \times \\
& \quad \times(\text { Number of } k \text {-valent vertices }) \times(\text { Cyclic ordering of edges at each vertex })
\end{aligned}
$$

We give its alternative definition in section 5 , and study examples.

In section 6 we briefly discuss how metric ribbon graphs appear in string theory.

\subsection{Why many formulae?}

Let us add some words to help the reader understand why many formulae are discussed in this paper.

If one is interested in computing specific $n$-point functions by Mathematica, the standard Wick rule is the most efficient operation. However, the Wick rule does not give an insight into the structure behind the $n$-point functions. In section 2 , we look for a concise formula, which expresses the sum over the bridge lengths $\left\{\ell_{i j}\right\}$ as a sum over permutations. We introduce extra degrees of freedom to make the formula concise, which makes it practically less efficient for computing concrete examples. The readers interested in comparing the efficiency can take a look at the attached supplementary material Mathematica files. ${ }^{4}$

The formulae in section 4 are neither practical nor concise. Our goal is to take the $n$-point functions, and find the geometric structure behind, namely the moduli space. This is a generalization of the work of [27] when there are multiple scalar fields. We notice that the $n$-point function splits into the flavor part and the color part. Since the moduli space carries the information about the color indices, what we need to do is to find the function $\mathscr{F}$ which reconstruct the information about the flavor indices. It turns out that the techniques developed in section 2 are useful for finding $\mathscr{F}$.

Our results are valid to all orders of the $1 / N_{c}$ expansion, but not at finite $N_{c}$. We do not take into account the fact that some operators become linearly dependent at finite $N_{c}$. In order to get the results exact in $N_{c}$, one should use the representation basis of operators, which is a finite-group Fourier transform of the permutation basis, explained in appendix A.2. The representation basis enables us to reproduce the matrix model results in $[10,41]$, which will be an important application of our method. ${ }^{5}$

\footnotetext{
${ }^{4}$ The file Tree $\mathrm{n}$-pt formula examples.nb implements the formulae in section 2 , and the file Tree $\mathrm{n}$-pt formula for graphs.nb explains the skeleton reduction in section 4 .

${ }^{5}$ The author thanks the referee of JHEP for bringing attention to these results.
} 


\section{Correlators from permutations}

We express tree-level $n$-point functions by permutations in various ways, and call them vertex-based, edge-based and face-based formulae. Basic techniques to study Wickcontractions by permutations will be explained below.

We define the permutation basis of scalar multi-trace operators by

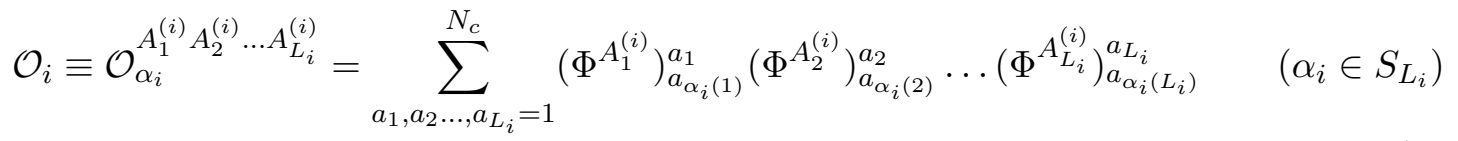

and impose the $\mathrm{U}\left(N_{c}\right)$ Wick rule (A.18) between scalar fields. ${ }^{6}$ Then we evaluate the correlator by the repeated application of the pairwise Wick-contractions as

$$
G_{n}=\left\langle\mathcal{O}_{1} \mathcal{O}_{2} \ldots \mathcal{O}_{n}\right\rangle=\sum_{\left\{\ell_{i j}\right\}} \prod_{i<j}^{n} \sum_{W_{i j}} \prod_{p=1}^{\ell_{i j}}\left[g^{A_{p}^{(i)} A_{q}^{(j)}} \delta_{b_{\alpha_{j}(q)}}^{a_{p}} \delta_{a_{\alpha_{i}(p)}}^{b_{q}}\right]_{q=W_{i j}^{-1}(p)}
$$

where $W_{i j}$ runs over the Wick-contractions at fixed bridge lengths. We rewrite r.h.s. by permutations.

\section{$2.1 \quad S_{2 L}$ (or vertex-based) formula}

Let us label the fields by $P=1,2, \ldots, 2 L$ and forget which index comes from which operator,

$$
\left\langle\mathcal{O}_{1} \mathcal{O}_{2} \ldots \mathcal{O}_{n}\right\rangle=\left\langle\prod_{i=1}^{n} \prod_{p=1}^{L_{i}}\left(\Phi^{A_{p}^{(i)}}\right)_{a_{\alpha_{i}(p)}}^{a_{p}}\left(x_{i}\right)\right\rangle \rightarrow\left\langle\mathcal{O}_{\text {all }}\right\rangle=\left\langle\prod_{P=1}^{2 L}\left(\Phi^{A_{P}}\right)_{a_{\alpha(P)}}^{a_{P}}\left(x_{P}\right)\right\rangle .
$$

Here $\alpha=\prod_{i=1}^{n} \alpha_{i} \in S_{2 L}$ is the permutation for all external operators, parameterized by

$$
\alpha_{k} \leftrightarrow \text { Permutation of }\left\{L_{k}^{\prime}+1, L_{k}^{\prime}+2, \ldots, L_{k+1}^{\prime}\right\}, \quad L_{k}^{\prime} \equiv \sum_{j=1}^{k-1} L_{j} .
$$

Denote the cycle type of $\alpha$ by $\lambda=\left[1^{\lambda_{1}} 2^{\lambda_{2}} \ldots\right] \vdash(2 L)$, where $\lambda_{K}$ is the number of singletrace operator of length $K$ in $\mathcal{O}_{\text {all }}$. Next we introduce a pairing map

$$
W_{0}:\{1,2, \ldots, 2 L\} \rightarrow\left\{\left(a_{1} a_{2}\right)\left(a_{3} a_{4}\right) \ldots\left(a_{2 L-1} a_{2 L}\right) \mid a_{P} \neq a_{Q}(P \neq Q)\right\} .
$$

This $W_{0}$ can be regarded as (the top element of $) \mathbb{Z}_{2}^{\otimes L}$,

$$
\left\{\prod_{k=1}^{L} \tau_{k}^{s_{k}} \mid \tau_{k}=\left(a_{2 k-1}, a_{2 k}\right), s_{k}=\{0,1\}\right\} \simeq \mathbb{Z}_{2}^{\otimes L}
$$

\footnotetext{
${ }^{6}$ Our notation will be explained further in appendix A.
} 

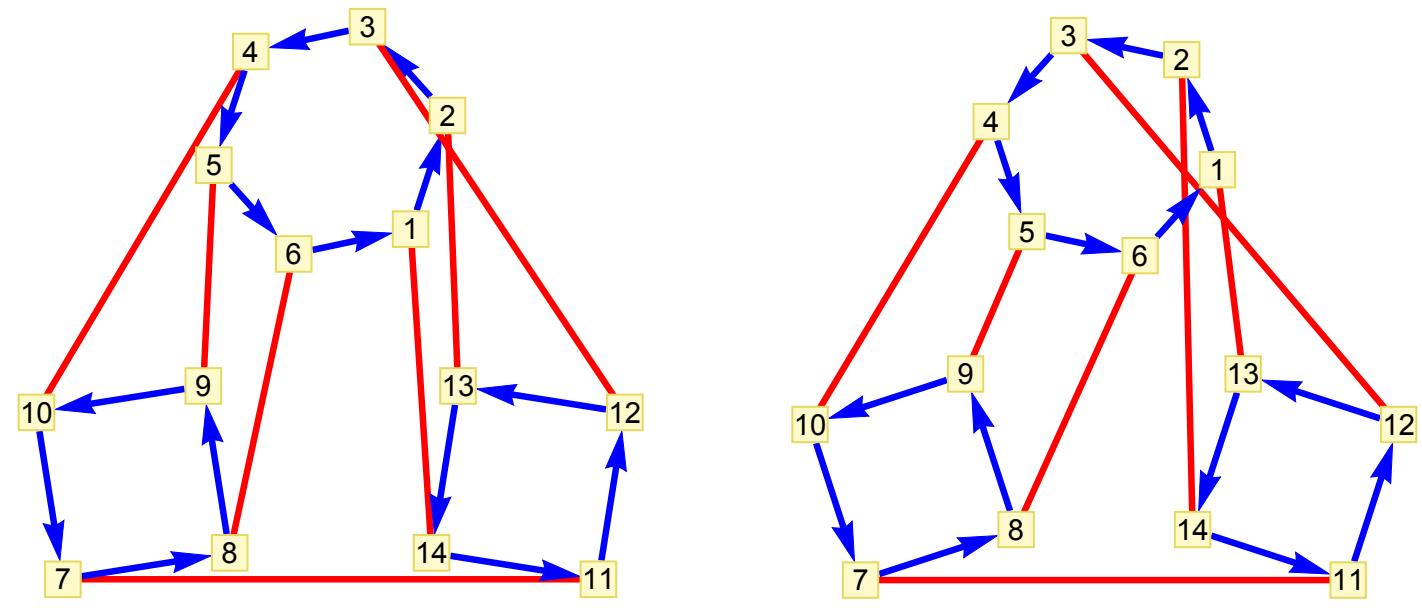

Figure 1. The Cayley graphs generated by $(\alpha, W)$ for $\left\langle\mathcal{O}_{1} \mathcal{O}_{2} \mathcal{O}_{3}\right\rangle$ with $\left(L_{1}, L_{2}, L_{3}\right)=(6,4,4)$. The left figure corresponds to a planar term, and the right figure to a non-planar term.

and we write $a_{2 k-1}=W_{0}\left(a_{2 k}\right)$ with $W_{0}^{2}=1$. By using (A.4), we permute $W_{0}$ by the adjoint action of $\gamma \in S_{2 L}$ to create all other choices of the subgroup $\mathbb{Z}_{2}^{\otimes L} \subset S_{2 L}$ as ${ }^{7}$

$$
W=\gamma W_{0} \gamma^{-1}=\prod_{k=1}^{L}\left(\gamma\left(a_{2 k-1}\right) \gamma\left(a_{2 k}\right)\right), \quad\left(\gamma \in S_{2 L}\right) .
$$

The above definition of $W$ is redundant, because it remains invariant under some relabeling of $\left\{a_{i}\right\}$. This redundancy is generated by the wreath-product group $S_{L}\left[\mathbb{Z}_{2}\right]$ as explained in appendix A. In contrast, our parameterization of $\mathcal{O}_{\text {all }}$ using $\alpha \in S_{2 L}$ has no redundancy, because we fix the order of the flavor indices $\left(A_{1}, A_{2}, \ldots, A_{2 L}\right)$. We can identify Feynman graphs with the Cayley graph generated by $\alpha \in S_{2 L}$ and $W \in \mathbb{Z}_{2}^{\otimes L}$, as shown in figure $1 .^{8}$

In the above notation, the original $n$-point formula (2.2) becomes

$$
G_{n}=\sum_{\left\{\ell_{i j}\right\}} \sum_{W} \prod_{P=1}^{2 L}\left[g^{A_{P} A_{W(P)}} \delta_{a_{\alpha W(P)}}^{a_{P}} \delta_{a_{\alpha(P)}}^{a_{W(P)}}\right]^{1 / 2}
$$

where we take the square root of $2 L$ factors, and used $W^{2}=1$. The $\delta$-functions can be simplified as

$$
\prod_{P=1}^{2 L} \delta_{a_{\alpha W(P)}}^{a_{P}} \prod_{Q=1}^{2 L} \delta_{a_{\alpha(Q)}}^{a_{W(Q)}}=N_{c}^{C(\alpha W)+C\left(\alpha W^{-1}\right)}=N_{c}^{2 C(\alpha W)}
$$

where $C(\omega)$ is defined in (A.8). It follows that

$$
G_{n}=\sum_{\left\{\ell_{i j}\right\}} \sum_{W}\left(\prod_{P=1}^{2 L}\left[g^{A_{P} A_{W(P)}}\right]^{1 / 2}\right) N_{c}^{C(\alpha W)} .
$$

\footnotetext{
${ }^{7}$ In the literature on graph theory and combinatorics, the adjoint action of $\gamma$ on edges is called an edge permutation.

${ }^{8}$ Strictly speaking, in Cayley graphs different colors should be used for different generators of the finite group. Our graphs are Cayley-like, because we use only two colors.
} 
This expression looks like a one-point formula on the permutation basis for any $n$.

Let $\mathcal{W}\left(\left\{\ell_{i j}\right\}\right)$ be the space of all pairing maps $W$ in (2.10), which satisfies the conditions

i) there are $\ell_{i j}$ contractions between $\mathcal{O}_{i}$ and $\mathcal{O}_{j}$.

ii) there are no self-contractions; $\ell_{i i}=0$.

Suppose $W_{0}$ is one such pairing, for example

$$
W_{0} \equiv W_{0}\left(\left\{\ell_{i j}\right\}\right)=\prod_{i<j} \prod_{p=1}^{\ell_{i j}}\left(L_{i, j}^{\prime}+p, L_{j, i}^{\prime}+p\right), \quad L_{i, j}^{\prime}=\sum_{k=1}^{i-1} L_{k}+\sum_{m=1}^{j-1} \ell_{i m}
$$

We can show that all other pairings are generated by the group action

$$
\mathcal{W}\left(\left\{\ell_{i j}\right\}\right)=\left\{\gamma W_{0} \gamma^{-1} \mid \gamma \in \prod_{i=1}^{n} S_{L_{i}}\right\} / S_{L}\left[\mathbb{Z}_{2}\right] .
$$

To see the equivalence, first we prove that r.h.s. does not change $\left\{\ell_{i j}\right\}$. Generally $W$ takes the form (2.7) under the constraint $\gamma \in \prod_{i=1}^{n} S_{L_{i}}$. Since any elements of $S_{L}$ is a product of transpositions, we consider $\gamma=\left(a a^{\prime}\right)$. The case of general $\gamma$ can be studied in a similar way. Because $\gamma \in \prod_{i=1}^{n} S_{L_{i}}$, both $a$ and $a^{\prime}$ must come from the same operator, say $\mathcal{O}_{a}$. Now

$$
\gamma=\left(a a^{\prime}\right): W_{0}=(a c)\left(a^{\prime} d\right) \mapsto \gamma W_{0} \gamma^{-1}=\left(a^{\prime} c\right)(a d), \quad\left(a, a^{\prime} \in \mathcal{O}_{a}, b \in \mathcal{O}_{b}, c \in \mathcal{O}_{c}\right)
$$

which does not change any of $\left(\ell_{a b}, \ell_{b c}, \ell_{c a}\right)$. Secondly, we argue that any $\gamma$ which preserves $\left\{\ell_{i j}\right\}$ should belong to $\prod_{i=1}^{n} S_{L_{i}}$. To see this, suppose that $\mathcal{O}_{i}$ and $\mathcal{O}_{j}$ are paired by $W_{0}$ and $W$ as

$$
W_{0} \ni \prod_{p=1}^{\ell_{i j}}\left(x_{p} y_{p}\right), \quad W \ni \prod_{p=1}^{\ell_{i j}}\left(x_{p}^{\prime} y_{p}^{\prime}\right) .
$$

The two permutations are related by $W \sim \gamma_{i j} W_{0} \gamma_{i j}^{-1}$, where

$$
\gamma_{i j}=\gamma_{i} \gamma_{j} \in S_{L_{i}} \times S_{L_{j}}, \quad x_{p}^{\prime}=\gamma_{i}\left(x_{p}\right), \quad y_{p}^{\prime}=\gamma_{j}\left(y_{p}\right)
$$

By repeating this argument to all $(i, j)$, one finds that $W=\gamma W_{0} \gamma^{-1}$ with $\gamma \in \prod_{i=1}^{n} S_{L_{i}}$.

Since $S_{L}\left[\mathbb{Z}_{2}\right]$ is not part of the group $\prod_{i=1}^{n} S_{L_{i}}$, the space $\mathcal{W}\left(\left\{\ell_{i j}\right\}\right)$ in (2.12) is not a group coset. For this reason, it is difficult to compute the sum over $W$ in (2.10).

In order to simplify the above formula further, we introduce the new inner-product,

$$
h^{A B}= \begin{cases}0 & \left(\text { self-contraction within } \mathcal{O}_{i}\right) \\ g^{A B} & \text { (otherwise) }\end{cases}
$$

We extend the space of all pairing maps $W$ as

$$
\bigsqcup_{i<j} \mathcal{W}\left(\left\{\ell_{i j}\right\}\right) \rightarrow \mathcal{W}=\left\{\gamma W_{0} \gamma^{-1} \mid \gamma \in S_{2 L} / S_{L}\left[\mathbb{Z}_{2}\right]\right\}
$$


where $W_{0} \in \mathbb{Z}_{2}^{\otimes L}$ is any set of $L$ pairs out of $2 L$ numbers without overlap like (2.5). Now the space $\mathcal{W}$ becomes a group coset. It follows that

$$
\begin{aligned}
G_{n} & =\sum_{\gamma \in S_{2 L} / S_{L}\left[\mathbb{Z}_{2}\right]}\left(\prod_{P=1}^{2 L}\left[h^{\left.A_{P} A_{\gamma W_{0} \gamma^{-1}(P)}\right]^{1 / 2}}\right) N_{c}^{C\left(\alpha \gamma W_{0} \gamma^{-1}\right)}\right. \\
& =\frac{1}{\left|\operatorname{Aut} W_{0}\right|} \sum_{\gamma \in S_{2 L}}\left(\prod_{P=1}^{2 L}\left[h^{A_{P} A_{\gamma W_{0} \gamma^{-1}(P)}}\right]^{1 / 2}\right) N_{c}^{C\left(\alpha \gamma W_{0} \gamma^{-1}\right)}
\end{aligned}
$$

where $\mid$ Aut $W_{0}|=| S_{L}\left[\mathbb{Z}_{2}\right] \mid=L ! 2^{L}$. The sum over $\left\{\ell_{i j}\right\}$ has been successfully removed.

\section{$2.2 \quad S_{L}$ (or edge-based) formula}

In the previous section, we labeled fields $\Phi^{A_{P}}$ by $P=1,2, \ldots, 2 L$ and derived formulae as a sum over $S_{2 L}$. Here we label Wick-contractions by $1,2, \ldots, L$, and give another formula in terms of $S_{L}$.

First, we extend the operator $\mathcal{O}_{i}$ by adding identity fields,

$$
\hat{\mathcal{O}}_{i} \equiv \mathcal{O}_{\alpha_{i}} \times \operatorname{tr}(\mathbf{1})^{L-L_{i}} \equiv \prod_{p=1}^{L}\left(\Phi^{\hat{A}_{p}^{(i)}}\right)_{a_{\hat{\alpha}_{i}(p)}}^{a_{p}}, \quad \hat{\alpha}_{i} \in S_{L}
$$

When $\Phi^{\hat{A}_{p}^{(i)}}=\mathbf{1}$, the permutation $\hat{\alpha}_{i}$ acts as the identity on $p$.

Second, we introduce $n$-tuple Wick-contraction by

$$
\left(\Phi^{\hat{A}_{1}}\right)_{a_{1}}^{b_{1}}\left(\Phi^{\hat{A}_{2}}\right)_{a_{2}}^{b_{2}}\left(\Phi^{\hat{A}_{3}}\right)_{a_{3}}^{b_{3}} \ldots\left(\Phi^{\hat{A}_{n}}\right)_{a_{n}}^{b_{n}}=h^{\hat{A}_{1} \hat{A}_{2} \ldots \hat{A}_{n}} \delta_{a_{1}}^{b_{2}} \delta_{a_{2}}^{b_{3}} \ldots \delta_{a_{1}}^{b_{n}} .
$$

We demand that $h^{\hat{A}_{1} \hat{A}_{2} \ldots \hat{A}_{n}}$ is equal to the two-point inner-product $g^{A B}$, if $(n-2)$ of the flavor indices $\left\{\hat{A}_{1}, \hat{A}_{2}, \ldots, \hat{A}_{n}\right\}$ are the identity field 1, and otherwise $h^{\hat{A}_{1} \hat{A}_{2} \ldots \hat{A}_{n}}=0$. For example, triple contraction is given by, ${ }^{9}$

$$
h^{A B C}=h^{A B} \delta_{\mathbf{1}}^{C}+h^{B C} \delta_{\mathbf{1}}^{A}+h^{C A} \delta_{\mathbf{1}}^{B}, \quad h^{A B}= \begin{cases}g^{A B} & (A \neq \mathbf{1}, B \neq \mathbf{1}) \\ 0 & (\text { otherwise })\end{cases}
$$

and in general

$$
h^{A_{1} A_{2} \ldots A_{n}}=\frac{1}{2(n-2) !} \sum_{\pi \in S_{n}} h^{A_{\pi(1)} A_{\pi(2)}} \prod_{j=3}^{n} \delta_{\mathbf{1}}^{A_{\pi(j)}} .
$$

With this definition of $h^{A_{1} A_{2} \ldots A_{n}}$, one can see that the $n$-tuple Wick-contraction (2.20) is equivalent to the pairwise Wick-contraction (see e.g. (2.2)), by recalling that $\hat{\alpha}_{i}(p)=p$ if $\Phi^{\hat{A}_{p}^{(i)}}=\mathbf{1}$.

Third, we evaluate the $n$-point functions of $\hat{\mathcal{O}}_{i}$ by taking all possible $n$-tuple Wickcontractions. One $n$-tuple Wick-contraction from $\left\langle\prod_{i} \hat{\mathcal{O}}_{i}\right\rangle$ can be specified by

$$
\left(W_{12}, W_{23}, \ldots, W_{n 1}\right) \in S_{L}^{\otimes n}, \quad W_{12} W_{23} \ldots W_{n 1}=1
$$

\footnotetext{
${ }^{9}$ We use the same symbol $h^{A B}$ as in (2.16), because both $h^{A B}$ 's remove the self-contractions.
} 
where $W_{i j} \in S_{L}$ tells which fields of $\hat{\mathcal{O}}_{i}$ and $\hat{\mathcal{O}}_{j}$ are paired together inside the $n$-tuple contraction. These permutations $\left\{W_{i j}\right\}$ satisfy $W_{i j} W_{j k}=W_{i k}$, and have the trivial monodromy $W_{i i}=1$. For example, three-point functions are given by

$$
\begin{aligned}
\left\langle\prod_{i=1}^{3} \hat{\mathcal{O}}_{i}\left(x_{i}\right)\right\rangle & \left.\equiv \sum_{W_{12}, W_{23}, W_{31}} \prod_{p, q, r=1}^{L} h^{\hat{A}_{p} \hat{B}_{q} \hat{C}_{r}} \delta_{a_{\hat{\alpha}_{1}(p)}}^{b_{q}} \delta_{\hat{\alpha}_{2}(q)}^{c_{r}} \delta_{c_{\hat{\alpha}_{3}(r)}}^{a_{p}}\right|_{p=W_{12}(q), q=W_{23}(r), r=W_{31}(p)} \\
& =\sum_{W_{12}, W_{23}, W_{31}}^{\prime} \prod_{p}^{L} h^{\hat{A}_{p} \hat{B}_{W_{12}-1(p)} \hat{C}_{W_{31}(p)}} \delta_{a_{\hat{\alpha}_{1} W_{12} \hat{\alpha}_{2} W_{23} \hat{\alpha}_{3} W_{31}(p)}^{a_{p}}} \\
& =\sum_{W_{12}, W_{23}, W_{31}}^{\prime}\left(\prod_{p}^{L} h^{\hat{A}_{p} \hat{B}_{W_{12}}^{-1}(p)} \hat{C}_{W_{31}(p)}\right) \delta_{L}\left(\Omega \hat{\alpha}_{1} W_{12} \hat{\alpha}_{2} W_{23} \hat{\alpha}_{3} W_{31}\right)
\end{aligned}
$$

where $\delta_{L}$ and $\Omega$ are defined in (A.5) and (A.24), respectively. The symbol $\sum^{\prime}$ means that the sums over $W_{12}, W_{23}, W_{31}$ are constrained by

$$
W_{12} W_{23} W_{31}=1 \in S_{L}
$$

We can solve the monodromy constraint formally by introducing

$$
W_{i j}=U_{i} U_{j}^{-1}, \quad U_{k} \in S_{L}
$$

Recall that $W_{i j}$ is a pairing function between $\mathcal{O}_{i}$ and $\mathcal{O}_{j}$. The new permutation $U_{i}$ acts only on $\hat{\alpha}_{i}$. The equation (2.24) becomes

$$
\begin{aligned}
\left\langle\prod_{i=1}^{3} \hat{\mathcal{O}}_{i}\left(x_{i}\right)\right\rangle= & \frac{1}{\left|S_{L}\right|} \sum_{U_{1}, U_{2}, U_{3} \in S_{L}}\left(\prod_{q=1}^{L} h^{\left.\hat{A}_{U_{1}(q)} \hat{B}_{U_{2}(q)} \hat{C}_{U_{3}(q)}\right)}\right. \\
& \times \delta_{L}\left(\Omega U_{1}^{-1} \hat{\alpha}_{1} U_{1} U_{2}^{-1} \hat{\alpha}_{2} U_{2} U_{3}^{-1} \hat{\alpha}_{3} U_{3}\right) .
\end{aligned}
$$

We introduced the factor $\left|S_{L}\right|=L$ ! because the simultaneous transformation $U_{k} \rightarrow U_{k} \gamma$ with $\forall \gamma \in S_{L}$ does not change $W_{i j}$ in (2.26).

The formula for general $n$-point functions of $\hat{\mathcal{O}}_{i}$ is

$$
\begin{aligned}
\left\langle\prod_{i=1}^{n} \hat{\mathcal{O}}_{i}\left(x_{i}\right)\right\rangle & =\frac{1}{L !} \sum_{\left\{U_{i}\right\} \in S_{L}^{\otimes n}}\left(\prod_{p=1}^{L} h^{\left.\check{A}_{p}^{(1)} \check{\check{A}}_{p}^{(2)} \ldots \check{A}_{p}^{(n)}\right)} \delta_{L}\left(\Omega \check{\alpha}_{1} \check{\alpha}_{2} \ldots \check{\alpha}_{n}\right)\right. \\
\check{A}_{p}^{(k)} & \equiv \hat{A}_{U_{k}(p)}^{(k)}, \quad \check{\alpha}_{k} \equiv U_{k}^{-1} \hat{\alpha}_{k} U_{k} .
\end{aligned}
$$

This quantity is proportional to the original correlator of $\left\{\mathcal{O}_{i}\right\}$. The proportionality constant comes from the permutations of the identity fields in (2.19). Since $\hat{\mathcal{O}}_{i}$ has $\left(L-L_{i}\right)$ identity fields, we find

$$
\left\langle\prod_{i=1}^{n} \mathcal{O}_{i}\left(x_{i}\right)\right\rangle=\frac{1}{\prod_{i=1}^{n}\left(L-L_{i}\right) !}\left\langle\prod_{i=1}^{n} \hat{\mathcal{O}}_{i}\left(x_{i}\right)\right\rangle .
$$




\subsubsection{Symmetry of the $S_{L}$ formula}

We summarize the symmetry of the formula (2.28).

First, the $n$-point formula is invariant under the simultaneous redefinition $\left\{U_{i}^{\prime}\right\}=$ $\left\{U_{i} \hat{\gamma}\right\}$. This corresponds to relabeling the Wick-contractions $p \rightarrow \hat{\gamma}(p)$ with $\hat{\gamma} \in S_{L}$. We can use this redundancy to set e.g. $U_{1}=1$.

Second, the extended operator (2.19) has the gauge symmetry

$$
\mathcal{O}_{\hat{\alpha}_{i}}^{\hat{A}_{1} \hat{A}_{2} \ldots \hat{A}_{L}}=\mathcal{O}_{\hat{\gamma}^{-1} \hat{\alpha}_{i} \hat{\gamma}}^{\hat{A}_{\hat{\gamma}(2)} \hat{A}_{\hat{\gamma}(2)} \hat{A}_{\hat{\gamma}(L)}}, \quad \hat{\gamma} \in S_{L_{i}} \times S_{L-L_{i}} \subset S_{L}
$$

generalizing (A.17). Our $n$-point formula is also invariant under the gauge transformations (2.30) for each fixed $i$, because the change $\left(\hat{\alpha}_{i}, \hat{A}_{p}^{(i)}\right) \rightarrow\left(\gamma_{i}^{-1} \hat{\alpha}_{i} \gamma_{i}, \hat{A}_{\gamma_{i}(p)}^{(i)}\right)$ can be absorbed by the redefinition $U_{i}^{\prime}=\gamma_{i} U_{i}$.

Third, the $n$-pt formula is invariant under any $S_{n}$ permutations,

$$
\left\langle\mathcal{O}_{1} \mathcal{O}_{2} \ldots \mathcal{O}_{n}\right\rangle=\left\langle\mathcal{O}_{\pi(1)} \mathcal{O}_{\pi(2)} \ldots \mathcal{O}_{\pi(n)}\right\rangle, \quad \forall \pi \in S_{n} .
$$

This follows from $S_{n}$ invariance of the $n$-tuple metric $h^{A_{1} A_{2} \ldots A_{n}}$ in (2.22), and the redefinition

$$
U_{k}^{-1} \hat{\alpha}_{k} U_{k} U_{k+1}^{-1} \hat{\alpha}_{k+1} U_{k+1}=V_{k+1}^{-1} \hat{\alpha}_{k+1} V_{k+1} U_{k}^{-1} \hat{\alpha}_{k} U_{k}, \quad\left(V_{k+1}^{-1}=U_{k}^{-1} \hat{\alpha}_{k} U_{k} U_{k+1}^{-1}\right) .
$$

In other words, two conjugacy classes commute. Recall that we introduced an artificial cyclic ordering $(12 \ldots n)$ in $(2.20)$, apparently breaking $S_{n}$ to $\mathbb{Z}_{n}$. We could use the $S_{n}$ invariant Wick-contraction rule,

$$
\delta_{i_{1}}^{j_{2}} \delta_{i_{2}}^{j_{3}} \ldots \delta_{i_{1}}^{j_{n}} \rightarrow \sum_{\pi \in \operatorname{Der}_{n}} \frac{1}{\left|\operatorname{Der}_{n}\right|} \delta_{\pi\left(i_{1}\right)}^{j_{1}} \delta_{\pi\left(i_{2}\right)}^{j_{2}} \ldots \delta_{\pi\left(i_{n}\right)}^{j_{n}},
$$

where $\operatorname{Der}_{n}$ is the derangement of $n$ elements (permutations without fixed points). The two definitions give the same result, because $h^{A_{1} A_{2} \ldots A_{n}}$ is locally a two-point inner-product, and the order of the color indices does not matter.

\subsubsection{Cayley graph}

We discuss how to draw a Cayley graph based on the $S_{L}$ formula. The main idea is to define an $n \times L$ Wick-contraction matrix

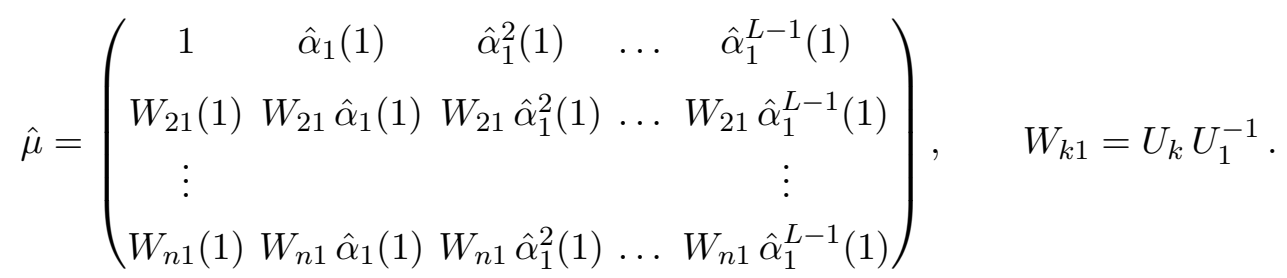

In general $\hat{\alpha}_{k}$ permutes the $k$-th row of $\hat{\mu}$ in a non-trivial way. We wrote the matrix $\hat{\mu}$ in the form where $\hat{\alpha}_{1}$ acts trivially. The $p$-th column of $\hat{\mu}$ corresponds to the $p$-th flavor factor

$$
\begin{aligned}
\left(\hat{\alpha}_{1}^{p-1}(1), W_{21} \hat{\alpha}_{1}^{p-1}(1), \ldots W_{n 1} \hat{\alpha}_{1}^{p-1}(1)\right) & \equiv\left(U_{1}(p), U_{2}(p), \ldots, U_{n}(p)\right) \\
& \leftrightarrow h^{\hat{A}_{U_{1}(p)}^{(1)} \hat{A}_{U_{2}(p)}^{(2)} \ldots \hat{A}_{U_{n}(p)}^{(n)}} .
\end{aligned}
$$




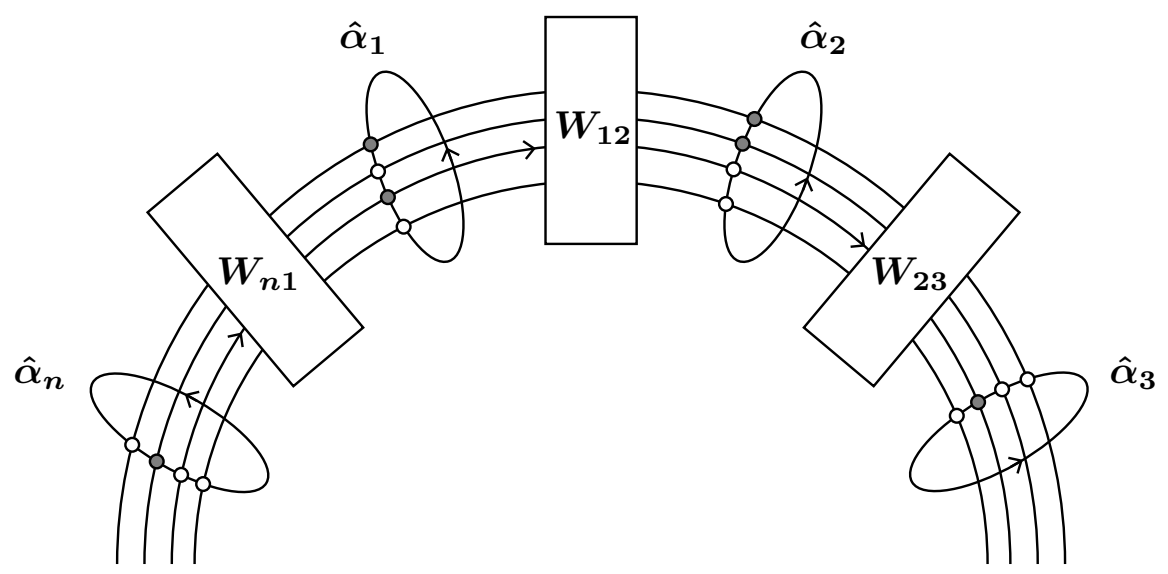

Figure 2. The Cayley graph in $S_{L}$ formula, generated by $\left\{\hat{\alpha}_{i}\right\}$ and $\left\{W_{i j}\right\}$. Gray circles represent the scalar fields, and white circles the identity fields.

Thanks to the monodromy constraint $W_{11}=1$, we can think of $\hat{\mu}$ as an element of $\mathbb{Z}_{n}^{\otimes L}$ consisting of $L$ columns. ${ }^{10}$ The two groups

$$
\hat{\alpha}=\otimes_{k=1}^{n} \hat{\alpha}_{k} \subset S_{L}^{\otimes n} \quad \text { and } \quad \hat{\mu} \in \mathbb{Z}_{n}^{\otimes L}
$$

can be used to draw a Cayley graph of the $n$-point function the $S_{L}$ formula, as in figure 2. This graph looks different from the Cayley graph in the $S_{2 L}$ formula. In particular, planar graphs in the $S_{2 L}$ formula may not be realized as a planar graph in the $S_{L}$ formula, even though the number of color cycles is the same for both.

Intuitively, the $n$-tuple Wick-contraction can be regarded as a hoop with two hooks. There are $L$ hoops in total. We hook the hoop at a pair of operators $\left(\mathcal{O}_{i}, \mathcal{O}_{j}\right)$, where each operator $\mathcal{O}_{i}$ has $L_{i}$ holes where one can hook the hoop. The position of the holes is permuted by $U$.

By knowing the position of the identity fields in $\hat{\mathcal{O}}_{i}$ from $(2.19)$, we can reconstruct the Wick-contraction structure immediately from $\hat{\mu}$. To see it, consider the following example. One possible Wick-contraction of $\left\langle\mathcal{O}_{1} \mathcal{O}_{2} \mathcal{O}_{3}\right\rangle$ with $\left(L_{1}, L_{2}, L_{3}\right)=(3,4,3)$ can be represented as

$$
\left\langle\operatorname{tr}\left(\Phi^{\left.A_{1} \Phi^{A_{2}} \Phi^{A_{3}}\right) \operatorname{tr}\left(\Phi^{B_{1}}\right.} \Phi^{B_{2}} \Phi^{\left.B_{3} \Phi^{B_{4}}\right) \operatorname{tr}\left(\Phi^{C_{1}} \Phi^{C_{2}} \Phi^{C_{3}}\right.}\right)\right\rangle \leftrightarrow\left(\begin{array}{llll}
A_{1} & A_{2} & A_{3} & \\
B_{1} & B_{2} & B_{3} & B_{4} \\
& C_{1} & C_{3} & C_{2}
\end{array}\right)
$$

\footnotetext{
${ }^{10}$ The matrix $\hat{\mu}$ has the symmetry $S_{n}^{\otimes L}$ if we sum over $\left\{U_{k}\right\}$. Here we consider a gauge-fixed version.
} 
We can slightly generalize the same matrix so that it has double columns, ${ }^{11}$

$$
\left(\begin{array}{ccccc}
A_{1} & A_{2} & A_{3} & \\
B_{1} & B_{2} & & B_{3} & B_{4} \\
& & C_{1} & C_{3} & C_{2}
\end{array}\right) \Leftarrow\left(\begin{array}{llllllll}
A_{1} & & A_{2} & & A_{3} & & & \\
& B_{1^{\prime}} & B_{2^{\prime}} & & B_{3} & B_{4} \\
& & & & C_{1^{\prime}} & C_{3^{\prime}} & C_{2^{\prime}}
\end{array}\right)
$$

This notation will be used later, e.g. in (2.59). To the empty entries, let us assign dummy indices $\left\{\hat{A}_{4}, \hat{A}_{5}, \hat{B}_{5}, \hat{C}_{4}, \hat{C}_{5}\right\}$ corresponding to $\mathbf{1}$ as

$$
\mu=\left(\begin{array}{ccccc}
A_{1} & A_{2} & A_{3} & \\
B_{1} & B_{2} & & B_{3} & B_{4} \\
& & C_{1} & C_{3} & C_{2}
\end{array}\right) \rightarrow\left(\begin{array}{lllll}
A_{1} & A_{2} & A_{3} & \hat{A}_{4} & \hat{A}_{5} \\
B_{1} & B_{2} & \hat{B}_{5} & B_{3} & B_{4} \\
\hat{C}_{4} & \hat{C}_{5} & C_{1} & C_{3} & C_{2}
\end{array}\right)=\hat{\mu} .
$$

We reconstruct the original Wick-contraction (2.37) by remembering the label of the dummy indices. The Wick contraction matrix plays an important rôle in the rest of the paper.

\subsubsection{Extremal case}

We show that the $n$-point function (2.28) in the extremal case $\sum_{i=1}^{n-1} L_{i}=L_{n}$ reduces to the two-point function (A.23). In the extremal correlator, all Wick contractions should be taken between $\mathcal{O}_{i}$ and $\mathcal{O}_{n}$ for $i=1,2, \ldots, n-1$. Thus

$$
L=\sum_{i=1}^{n-1} \ell_{i n}=\sum_{i=1}^{n-1} L_{i}=L_{n}
$$

Using the gauge symmetry, we may fix the position of the scalar and identity fields of $\hat{\mathcal{O}}_{i}$ as

$$
\Phi^{\hat{A}_{p}^{(i)}}=1 \quad \text { unless } \quad L_{i}^{\prime} \leq p<L_{i+1}^{\prime}, \quad L_{i}^{\prime}=\sum_{k=1}^{i-1} \ell_{k n}, \quad L_{1}^{\prime}=0
$$

and assume that $\alpha_{i} \in S_{L_{i}}$ acts on the range $L_{i}^{\prime} \leq p<L_{i+1}^{\prime}$. We can restrict the sum over $\left\{U_{i}\right\} \in S_{L}^{\otimes n}$ in $(2.28)$ as

$$
\left(U_{1}, U_{2}, \ldots U_{n-1}, U_{n}\right) \quad \rightarrow \quad\left(u_{1}, u_{2}, \ldots u_{n-1}, U_{n}\right) \in\left(S_{L_{1}}, S_{L_{2}}, \ldots, S_{L_{n-1}}, S_{L}\right) .
$$

Then the flavor factor simplifies as

$$
\prod_{p=1}^{L} h^{\check{A}_{p}^{(1)} \check{A}_{p}^{(2)} \ldots \check{A}_{p}^{(n)}}=\left(\prod_{p=L_{i}^{\prime}+1}^{L_{i+1}^{\prime}} \frac{g^{A_{u_{i}(p)}^{(i)} A_{U_{n}(p)}^{(n)}}}{\left|x_{i}-x_{n}\right|^{2}}\right) \equiv \prod_{q=1}^{L} \tilde{g}^{B_{V U_{n}^{-1}(q)}} A_{q}^{(n)}
$$

\footnotetext{
${ }^{11}$ Since the flavor inner-product $g^{A B}$ is symmetric, we do not distinguish two ways to double the columns, i.e.

$$
\left(\begin{array}{l}
A \\
B
\end{array}\right) \sim\left(\begin{array}{cc}
A & \\
B
\end{array}\right) \sim\left(\begin{array}{c}
A \\
B
\end{array}\right)
$$
}


with $p=U_{n}(q)$, and the product of $\alpha_{i}$ can be unified as

$$
\begin{aligned}
& u_{1}^{-1} \alpha_{1} u_{1} u_{2}^{-1} \alpha_{2} u_{2} \ldots u_{n-1}^{-1} \alpha_{n-1} u_{n-1}=V^{-1} \beta V \in S_{L}, \\
& \quad V=u_{1} \circ u_{2} \circ \cdots \circ u_{n-1}, \quad \beta=\alpha_{1} \circ \alpha_{2} \circ \cdots \circ \alpha_{n-1} \in \prod_{i=1}^{n-1} S_{L_{i}} \subset S_{L} .
\end{aligned}
$$

By writing $V_{n} \equiv V U_{n}^{-1}$, we find that one of the sums over $V$ and $U_{n}$ is trivial. The $n$-point function becomes

$$
\left\langle\prod_{i=1}^{n} \hat{\mathcal{O}}_{i}\left(x_{i}\right)\right\rangle=\sum_{V_{n} \in S_{L}}\left(\prod_{p=1}^{L} \tilde{g}^{B} V_{n}^{-1(p)} A_{p}^{(n)}\right) \delta_{L}\left(\Omega \beta V_{n} \alpha_{n} V_{n}^{-1}\right)
$$

which agrees with the two-point function (A.23).

\subsection{Face-based formulae}

From a Cayley graph generated by $(\alpha, W)$, we define the dual graph by

$$
\text { Dual : }(\alpha, W) \mapsto(\omega, W), \quad \omega \equiv \alpha W .
$$

We want to express the $n$-point functions as a sum over the face permutation $\omega$, instead of a sum over $W$,

$$
G_{n}=\sum_{\omega} \mathbb{F}(\omega)
$$

where $\mathbb{F}$ computes the color and flavor factors associated to $\omega$.

In order to define $\mathbb{F}$, we need to parametrize the faces of the graph. The Feynman graphs in the double-line notation have two types of edges, those from Wick-contractions and the others from external operators. The red lines in figure 1 signify the former, and the blue ones signify the latter. We call the edge from Wick-contractions the Wick-edge, and the edge from external operators the rim. Each Wick-edge is connected to a pair of rims, and each rim is oriented by $\alpha=\prod_{i=1}^{n} \alpha_{i} \in S_{2 L}$.

There are two ways to label rims, depending on whether one simplifies $\alpha$ or $W$. In the first method, we label the rims by $\{1,2, \ldots, 2 L\}$ as in figure 3 . These numbers are ordered in the same way as $\alpha$ explained in (2.4). ${ }^{12}$ In the second method, we label the Wick-edges by $\left\{1,1^{\prime}, 2,2^{\prime}, \ldots, L, L^{\prime}\right\}$. The Wick-contraction is given by $W=\prod_{p}\left(p p^{\prime}\right)$. Since the whole graph is oriented, each rim starts from a Wick-edge and ends at another Wick-edge. We label the rim by using the ending Wick-edge as in figure 4 .

Rim-labels by $\boldsymbol{W}$. We consider $n$-point functions of single-trace operators by using the rims labeled by $W$. The rim-labels by $\alpha$ will be studied in appendix B. For simplicity, we assume that all external operators are single-traces.

To begin with, we show the equivalence between the set of unlabeled graphs and face permutations. The face permutation should not have one-cycles, because the one-cycles come from self-contractions. We define $S_{2 L}^{\times} \subset S_{2 L}$ by

$$
\omega \in S_{2 L}^{\times} \quad \Leftrightarrow \quad p \neq \omega(p) \quad(\forall p) .
$$

\footnotetext{
${ }^{12}$ For example, $\alpha_{2}$ contains $L_{2}$ rims labeled by $L_{1}+1, L_{1}+2, \ldots, L_{1}+L_{2}$.
} 

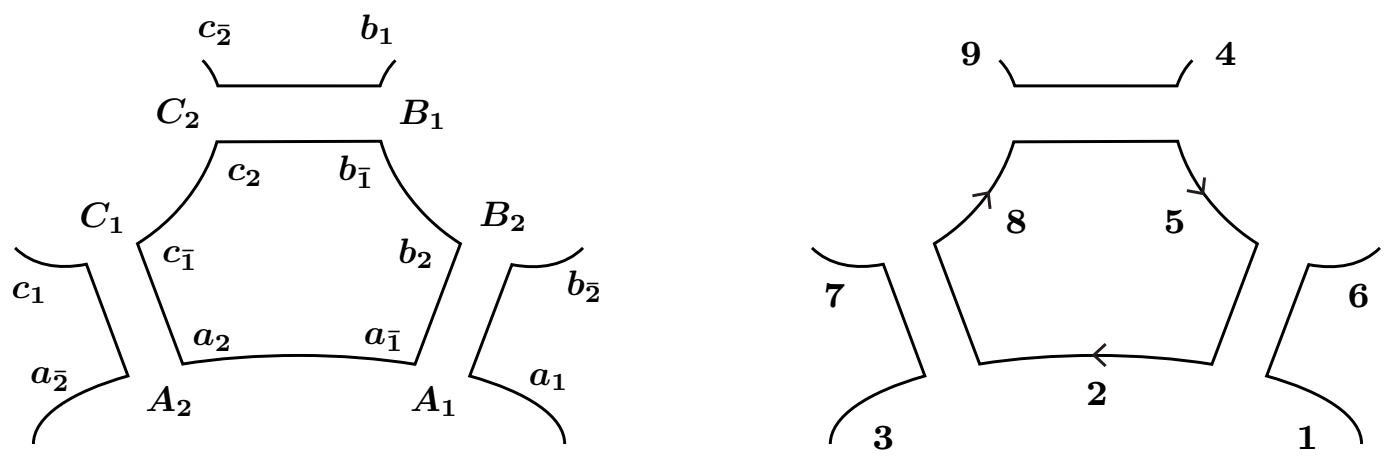

Figure 3. (Left) Wick-contraction in the half-edge notation, see appendix A.3. This is the Cayley graph generated by $\iota_{\alpha}=\left(a_{1^{\prime}} a_{2}\right)\left(b_{1^{\prime}} b_{2}\right)\left(c_{1^{\prime}} c_{2}\right) \ldots$ and $W=\left(A_{1} B_{2}\right)\left(B_{1} C_{2}\right)\left(C_{1} A_{1}\right) \ldots$ (Right) The same graph whose rim between $a_{\bar{P}}$ and $a_{\alpha(P)}$ is labeled as $P$.
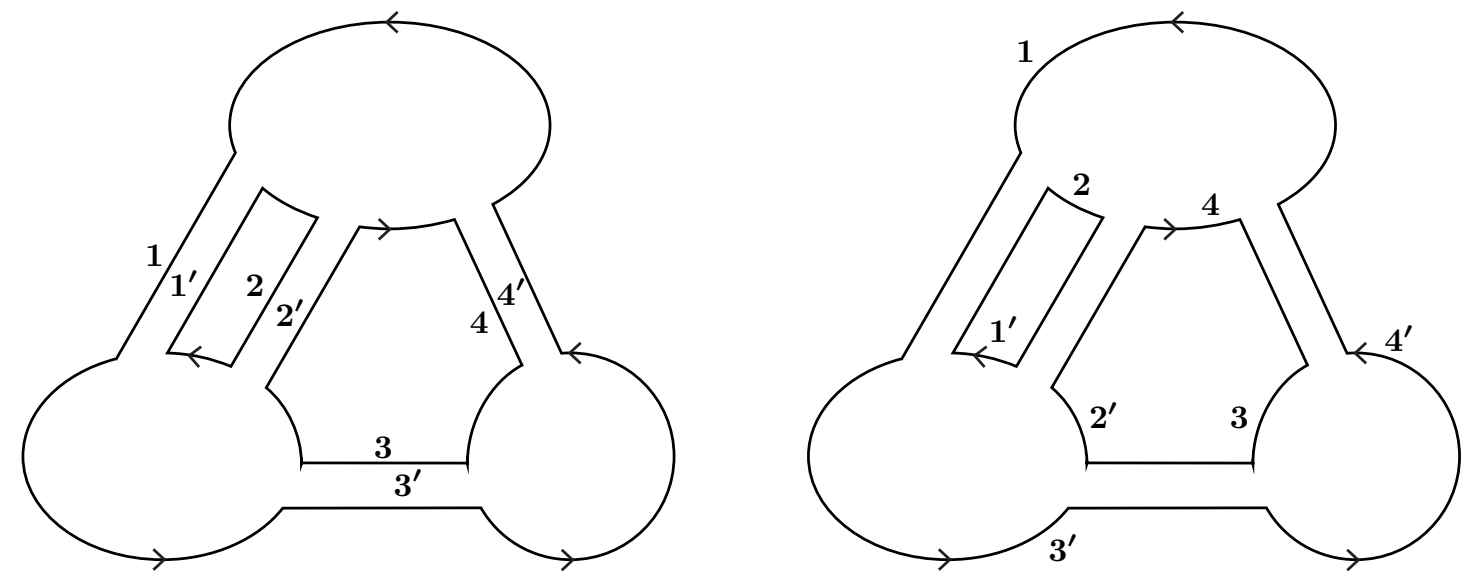

Figure 4. (Left). Wick-contraction in the double-line notation with the numbers $\left\{1,1^{\prime}, 2,2^{\prime}, \ldots\right\}$ labeling Wick-edges. (Right). The same graph whose rims are labeled. Both figures correspond to $\omega=\left(1^{\prime} 2\right)\left(2^{\prime} 43\right)\left(13^{\prime} 4^{\prime}\right)$.

It follows that

$$
\text { \{Feynman graphs with } 2 L \text { unlabeled vertices }\} \quad \leftrightarrow \omega \in S_{2 L}^{\times} / S_{L}\left[\mathbb{Z}_{2}\right] .
$$

In r.h.s., we define $\omega$ as the permutation of $\left\{1,1^{\prime}, 2,2^{\prime}, \ldots, L, L^{\prime}\right\}$ modulo the action of $S_{L}\left[\mathbb{Z}_{2}\right]$ explained in (A.11). Owing to the redundancy of relabeling, $\omega$ is in one-to-one correspondence with the coset $S_{2 L}^{\times} / S_{L}\left[\mathbb{Z}_{2}\right]$, where the denominator has the adjoint action like (2.17). We have already explained how to construct a face permutation from a given graph in the double-line notation; label the Wick edges using $\left\{1,1^{\prime}, 2,2^{\prime}, \ldots, L, L^{\prime}\right\}$ and export them to rims. Conversely, we can construct a graph from the face permutation as follows. Consider the permutation $\alpha_{\bullet} \equiv \omega W_{\bullet} \in S_{2 L}$ with

$$
W_{\bullet} \equiv \prod_{p=1}^{L}\left(p p^{\prime}\right) \text {. }
$$


If we regard $\alpha_{\bullet}$ as the permutation for the external single-trace operators, we can make all rims oriented. By connecting the end-points of the rim pairs $p, p^{\prime}$, we obtain a Feynman diagram in the double-line notation. Hence the relation (2.49) is proven.

We make two more comments. First, the relation (2.49) tells nothing about the cycle type of $\alpha_{\bullet}$. To compute $G_{n}$ we need to restrict the sum over $\omega$ further. Second, in this construction $\omega$ produces an unlabeled graph as exemplified in figure 4 . In other words, the rim labels $\left\{1,1^{\prime}, 2,2^{\prime}, \ldots, L, L^{\prime}\right\}$ are not related to the flavor indices of the external operators, and the identification is not unique. Generally, an unlabeled graph is an equivalence class of the relabeling group acting on labeled graphs. Thus, the function $\mathbb{F}$ in (2.47) is a map from a set of graphs to a polynomial of $\left(N_{c}, g^{A B}\right)$, where the graph set is a certain group orbit.

We define $\mathbb{F}$ in the following way. To begin with, we interpret a permutation as a set of ordered lists. For example, a permutation of the cycle type $\left[2^{2}\right]$ can be interpreted as eight ordered lists,

$$
\begin{aligned}
S_{4} \ni(12)\left(1^{\prime} 2^{\prime}\right) \stackrel{\mathbf{C L}}{\longleftrightarrow}\left\{(1,2)\left(1^{\prime}, 2^{\prime}\right),(2,1)\left(1^{\prime}, 2^{\prime}\right),(1,2)\left(2^{\prime}, 1^{\prime}\right),(2,1)\left(2^{\prime}, 1^{\prime}\right),\right. \\
\left.\left(1^{\prime}, 2^{\prime}\right)(1,2),\left(1^{\prime}, 2^{\prime}\right)(2,1),\left(2^{\prime}, 1^{\prime}\right)(1,2),\left(2^{\prime}, 1^{\prime}\right)(2,1)\right\}
\end{aligned}
$$

where $\mathbf{C L}$ stands for the map between cycles to lists. Generally, a permutation of the cycle type $\lambda=\left[1^{\lambda_{1}} 2^{\lambda_{2}} \ldots\right]$ can be interpreted as $|\mathbf{C L}(\lambda)|$ ordered lists, where

$$
|\mathbf{C L}(\lambda)|=\left|\prod_{k} S_{\lambda_{k}}\left[\mathbb{Z}_{k}\right]\right|=\prod_{k}\left(\lambda_{k} ! k^{\lambda_{k}}\right) .
$$

The multiple interpretations come from the translation of each cycle and permuting the cycles of the same length. In other words, $\mathbf{C L}\left(\alpha_{\bullet}\right)$ is isomorphic to the stabilizer subgroup, ${ }^{13}$

$$
\operatorname{Stab}\left(\alpha_{\bullet}\right) \equiv\left\{\gamma \in S_{2 L} \mid \gamma \alpha_{\bullet} \gamma^{-1}=\alpha_{\bullet}\right\}
$$

Indeed, if $\alpha_{\bullet}$ has the cycle type $\lambda=\left[1^{\lambda_{1}} 2^{\lambda_{2}} \ldots\right]$, the order of $\operatorname{Stab}\left(\alpha_{\bullet}\right)$ is ${ }^{14}$

$$
\left|\operatorname{Stab}\left(\alpha_{\bullet}\right)\right|=\prod_{k} S_{\lambda_{k}}\left[\mathbb{Z}_{k}\right]=|\mathbf{C L}(\lambda)|
$$

Given the permutation $\alpha_{\bullet}$, we pick up one element from $\mathbf{C L}\left(\alpha_{\bullet}\right)$ and write it as $\beta \equiv\left(\beta_{1}, \ldots, \beta_{n}\right) \cdot{ }^{15}$ In the example of figure 4 , we find $\alpha=(124)\left(1^{\prime} 3^{\prime} 2^{\prime}\right)\left(34^{\prime}\right)$. Let us choose an ordered list as

$$
\beta=\left(1^{\prime}, 3^{\prime}, 2^{\prime}\right)(2,4,1)\left(3,4^{\prime}\right) \in \mathbf{C L}(\alpha) .
$$

The choice of $\beta$ picks up a Feynman graph, i.e. one set of Wick-contractions appearing in

$$
\left\langle\mathcal{O}_{1} \mathcal{O}_{2} \mathcal{O}_{3}\right\rangle=\left\langle\operatorname{tr}\left(\Phi^{A_{1}} \Phi^{A_{2}} \Phi^{A_{3}}\right) \operatorname{tr}\left(\Phi^{A_{4}} \Phi^{A_{5}} \Phi^{A_{6}}\right) \operatorname{tr}\left(\Phi^{A_{7}} \Phi^{A_{8}}\right)\right\rangle
$$

\footnotetext{
${ }^{13}$ In the literature, $\operatorname{Stab}\left(\alpha_{\bullet}\right)$ is also called the automorphism group $\operatorname{Aut}\left(\alpha_{\bullet}\right)$.

${ }^{14}$ Since all operators are assumed to be single-traces, $\lambda_{k}$ is the number of single-trace operators of length $k$.

${ }^{15}$ At this point $n$ is a free parameter. We will equate $n$ with the number of external single-trace operators in $(2.65)$.
} 
Let us make this point clearer. The ordered list $\beta \in \mathbf{C L}\left(\alpha_{\bullet}\right)$ relates the rim labels to the flavor indices as

$$
\beta_{i}:\left\{1,1^{\prime}, 2,2^{\prime}, \ldots\right\} \rightarrow\left\{0,1,2, \ldots, L_{i}\right\}
$$

where $\beta_{i}^{-1}(x)$ is uniquely defined if and only if $x \neq 0 .{ }^{16}$ We parameterize $\alpha$ as

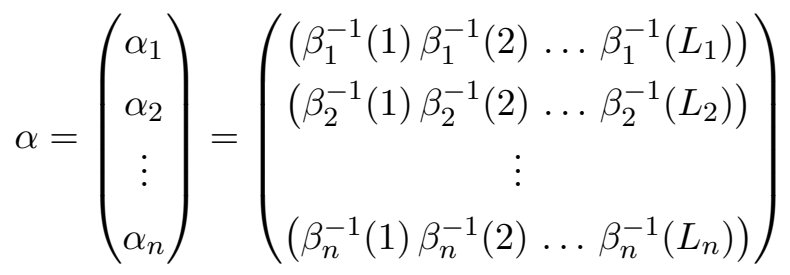

and construct the Wick-contraction matrix (see (2.38)),

$$
\hat{\mu}_{\beta}=\left(\begin{array}{cccccc}
\mu_{1,1} & \mu_{1,1^{\prime}} & \mu_{1,2} & \mu_{1,2^{\prime}} & \ldots & \mu_{1, L^{\prime}} \\
\mu_{2,1} & \mu_{2,1^{\prime}} & \mu_{2,2} & \mu_{2,2^{\prime}} & \ldots & \mu_{2, L^{\prime}} \\
\vdots & & & & & \\
\mu_{n, 1} & \mu_{n, 1^{\prime}} & \mu_{n, 2} & \mu_{n, 2^{\prime}} & \ldots & \mu_{n, L^{\prime}}
\end{array}\right), \quad \mu_{i, s}= \begin{cases}A_{\beta_{i}(s)}^{(i)} & \left(\beta_{i}(s) \neq 0\right) \\
\mathbf{1} & \left(\beta_{i}(s)=0\right) .\end{cases}
$$

The matrix $\hat{\mu}_{\beta}$ is defined uniquely for each $\beta \in \mathbf{C L}\left(\alpha_{\bullet}\right)$. We associate a flavor factor to $\hat{\mu}_{\beta}$ as

$$
\mathbf{F}\left(\hat{\mu}_{\beta}\right)=\prod_{s=1}^{L} h^{\mu_{1, s} \mu_{1, s^{\prime}} \mu_{2, s} \mu_{2, s^{\prime}} \ldots \mu_{n, s} \mu_{n, s^{\prime}}}
$$

where $h^{\mu_{1, s} \mu_{1, s^{\prime}} \cdots}$ is defined by

$$
h^{A_{1} A_{1^{\prime}} \ldots A_{n} A_{n^{\prime}}}=\frac{1}{2(2 n-2) !} \sum_{\pi \in S_{2 n}} h^{A_{\pi(1)} A_{\pi\left(1^{\prime}\right)}} \prod_{j=2}^{n} \delta_{\mathbf{1}}^{A_{\pi(j)}} \delta_{\mathbf{1}}^{A_{\pi\left(j^{\prime}\right)}}
$$

like (2.22), and satisfies

$$
h^{\mu_{1, s} \mu_{1, s^{\prime}} \mu_{2, s} \mu_{2, s^{\prime}} \ldots \mu_{n, s} \mu_{n, s^{\prime}}}=h^{A_{s}^{\left(i_{s}\right)} A_{s^{\prime}}^{\left(j_{s}\right)}} \quad \text { for some }\left(i_{s}, j_{s}\right) \text { from } 1,2, \ldots, n .
$$

The permutation $S_{2 n}$ in (2.61) runs over two columns of $\hat{\mu}_{\beta}$, where two out of $2 n$ entries should not be 1. From (2.62) we obtain the set of $L$ pairs

$$
\left\{\left(A_{1}^{\left(i_{1}\right)}, A_{1^{\prime}}^{\left(j_{1}\right)}\right)\left(A_{2}^{\left(i_{2}\right)}, A_{2^{\prime}}^{\left(j_{2}\right)}\right) \ldots\left(A_{L}^{\left(i_{L}\right)}, A_{L^{\prime}}^{\left(j_{L}\right)}\right)\right\}
$$

which is essentially $W \in \mathbb{Z}_{2}^{\otimes L}$ in section 2.1. The self-contractions are removed by the property of $h^{A B}$ in (2.16).

We should sum over the choices $\beta \in \mathbf{C L}\left(\alpha_{\bullet}\right)$ entering in $\hat{\mu}_{\beta}$. This procedure corresponds to labeling unlabeled graphs. Then we define the function on faces by

$$
\mathbb{F}(\omega)=N_{c}^{C(\omega)} \sum_{\beta \in \operatorname{Stab}\left(\alpha_{\bullet}\right)} \mathbf{F}\left(\hat{\mu}_{\beta}\right)
$$

\footnotetext{
${ }^{16}$ Here $x=0$ implies that the corresponding rim label is not assigned to $\mathcal{O}_{i}$.
} 
with $\mathbf{F}\left(\hat{\mu}_{\beta}\right)$ in (2.60). Using the correspondence (2.49), we can write the $n$-point function of single-trace operators as

$$
G_{n}=\frac{1}{\left|S_{L}\left[\mathbb{Z}_{2}\right]\right|} \sum_{\omega \in S_{2 L}^{\times}} \mathbb{F}(\omega) \delta_{\text {cycle-type }}\left(\alpha_{\text {ex }}^{-1}, \omega W_{\bullet}\right) .
$$

We inserted the $\delta$-function in order to guarantee that $\alpha_{\bullet}=\omega W_{\bullet}$ has the same cycle type of $\alpha_{\text {ex }}$ specified by the external operators. We rewrite (2.65) in terms of the conjugacy class (A.6) of $S_{2 L}$ as

$$
G_{n}=\frac{1}{\left|S_{L}\left[\mathbb{Z}_{2}\right]\right|} \frac{(2 L) !}{\left|\operatorname{Stab}\left(\alpha_{\mathrm{ex}}\right)\right|} \sum_{\omega \in S_{2 L}^{\times}} \mathbb{F}(\omega) \delta_{2 L}\left(\left[\alpha_{\mathrm{ex}}^{-1}\right] \omega W_{\bullet}\right) .
$$

We checked this formula by Mathematica for simple cases.

\section{Pants decomposition}

Let us discuss the geometric interpretation of the $n$-point function in the $S_{L}$ formalism. We rewrite the formula (2.28) as a product of three-point functions, or pairs of pants, schematically as

$$
\begin{aligned}
\mathcal{X}\left[\Sigma_{0, n}\right] & \equiv \frac{1}{L !} \sum_{U}\left(\prod_{p=1}^{L} h^{\check{A}_{p}^{(1)} \check{A}_{p}^{(2)} \ldots \check{A}_{p}^{(n)}}\right) \delta_{L}\left(\Omega \check{\alpha}_{1} \check{\alpha}_{2} \ldots \check{\alpha}_{n}\right) \\
& \sim \operatorname{Glue}\left(\mathcal{X}\left[\mathcal{S}_{1}\right], \mathcal{X}\left[\mathcal{S}_{2}\right], \ldots, \mathcal{X}\left[\mathcal{S}_{n-2}\right]\right)
\end{aligned}
$$

where $\left\{\mathcal{S}_{f}\right\}$ defines a pants decomposition of $\mathbb{C P}_{1}$ with $n$ punctures,

$$
\Sigma_{0, n}=\mathcal{S}_{1} \amalg \mathcal{S}_{2} \amalg \cdots \amalg \mathcal{S}_{n-2} \cdot
$$

The last equation of (3.1) will be made precise in (3.12). This expression implies that the tree-level $n$-point function is a certain topological partition function on $\mathbb{C P}_{1}$ with $n$ boundaries and defects. We call it topological, because it is invariant under different pants decompositions, and does not depend on the complex structure of $\Sigma_{0, n} \cdot{ }^{17}$ The punctures represent external operators $\left\{\hat{\mathcal{O}}_{i}\right\}$, and each factor $\mathcal{X}\left[\mathcal{S}_{f}\right]$ represents a local three-point interaction. The defects carry all the information about $N_{c}$. This is why higher-genus Riemann surfaces do not show up. The sum over $U$ 's in (3.1) can be thought of as a sum over different twisted sectors, or the partition function of a topological gauge theory with finite gauge groups $[42-45] .{ }^{18}$

To derive (3.1), consider the color and flavor factors separately.

The color factor decomposes as follows. Thanks to the permutation symmetry (2.31), without loss of generality we may assume $\mathcal{S}_{1}$ contains $\left\{\alpha_{1}, \alpha_{2}\right\}, \mathcal{S}_{k}$ contains $\alpha_{k+1}$ for

\footnotetext{
${ }^{17}$ We can also think of $\Sigma_{0, n}$ as $\mathbb{C P}_{1}$ with $n$ boundaries, because the topological partition function does not depend on the length of boundary circles.

${ }^{18}$ Beware that (3.1) is not the topological string partition function on $\mathbb{P}^{1}$ mentioned in Introduction.
} 


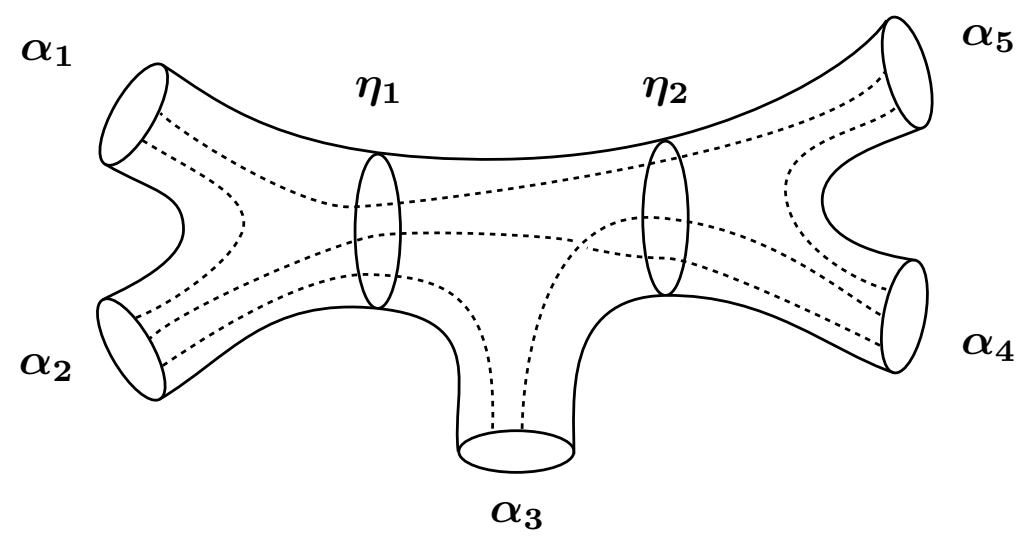

Figure 5. Pants decomposition of the five-point function $\left\langle\mathcal{O}_{1} \mathcal{O}_{2} \mathcal{O}_{3} \mathcal{O}_{4} \mathcal{O}_{5}\right\rangle$, with intermediate states denoted by $\mathcal{O}_{\eta_{1}}$ and $\mathcal{O}_{\eta_{2}}$. Although this figure has a fixed number of bridge lengths, the sum over $\left\{\ell_{i j}\right\}$ has already been performed in our formula.

$2 \leq k \leq n-3$, and $\mathcal{S}_{n-2}$ has $\left\{\alpha_{n-1}, \alpha_{n}\right\}$. Then we insert the resolution of identity on the permutation basis ${ }^{19}$

$$
\delta_{L}(\alpha \beta)=\sum_{\eta \in S_{L}} \delta_{L}(\alpha \eta) \delta_{L}\left(\eta^{-1} \beta\right)
$$

Now we rewrite the $\delta$-function as

$$
\begin{aligned}
\delta_{L}\left(\Omega \check{\alpha}_{1} \check{\alpha}_{2} \ldots \check{\alpha}_{n}\right)= & \sum_{\omega_{1}, \omega_{2}, \ldots, \omega_{n-2} \in S_{L}} \sum_{\eta_{1}, \eta_{2}, \ldots, \eta_{n-3} \in S_{L}} \delta_{L}\left(\Omega\left[\omega_{1}\right]\left[\omega_{2}\right] \ldots\left[\omega_{n-2}\right]\right) \times \\
& \times \delta_{L}\left(\omega_{1}^{-1} \check{\alpha}_{1} \check{\alpha}_{2} \eta_{1}\right) \prod_{k=2}^{n-3} \delta_{L}\left(\omega_{k}^{-1} \eta_{k-1}^{-1} \check{\alpha}_{k+1} \eta_{k}\right) \delta_{L}\left(\omega_{n-2}^{-1} \eta_{n-3}^{-1} \check{\alpha}_{n-1} \check{\alpha}_{n}\right)
\end{aligned}
$$

where $\left[\omega_{k}\right]$ is the conjugacy class defined in (A.6). Since two conjugacy classes commute, the first line is manifestly invariant under the permutation of different $\omega$ 's. This decomposition is similar to the following set of OPE's, ${ }^{20}$

$$
\mathcal{O}_{1} \times \mathcal{O}_{2} \rightarrow \mathcal{O}_{\eta_{1}}, \quad \mathcal{O}_{\eta_{k-1}} \times \mathcal{O}_{k+1} \rightarrow \mathcal{O}_{\eta_{k}} \quad(2 \leq k \leq n-3), \quad \mathcal{O}_{n-3} \times \mathcal{O}_{n-1} \rightarrow \mathcal{O}_{n}
$$

We identify the $\delta$-function $\delta_{L}\left(\omega_{k}^{-1} \eta_{k-1}^{-1} \check{\alpha}_{k+1} \eta_{k}\right)$ as the color part of $\mathcal{X}\left[\mathcal{S}_{k}\right]$. This structure is also depicted in figure 5.

In order to split the flavor part of the formula (3.1), we introduce the functions

$$
H^{A_{1} A_{2} A_{3}}(\phi)=\phi^{-1} h^{A_{1} A_{2} A_{3}}+\prod_{j=1}^{3} \delta_{\mathbf{1}}^{A_{j}}, \quad \zeta_{A B}(\phi)=\phi g_{A B}+\delta_{A}^{\mathbf{1}} \delta_{B}^{\mathbf{1}}
$$

\footnotetext{
${ }^{19}$ This relation is not the same as the resolution of identity in the Hilbert space of gauge theory. In general, the inverse of tree-level two-point functions is much more complicated than the inverse of permutations.

${ }^{20}$ Strictly speaking, this operation is not OPE. Generally, an OPE produces several terms, while inserting the resolution of identity produces one term.
} 
where $\phi$ is an extra parameter to count the scalars (i.e. non-identity fields), and $g_{A B}$ is the inverse metric

$$
g^{A B} g_{B C} g^{C D}=g^{A D}, \quad g^{11}=g_{11}=0 .
$$

According to (2.21), $h^{A_{1} A_{2} \ldots A_{n}} \neq 0$ when it has precisely two scalars. Thus $H^{A_{1} A_{2} A_{3}} \neq 0$ when it has zero or two scalar indices.

By using (3.6) and (3.7), we find the identity

$$
\begin{aligned}
& \prod_{p=1}^{L} h^{\check{A}_{p}^{(1)} \check{A}_{p}^{(2)} \ldots \check{A}_{p}^{(n)}}=\prod_{p=1}^{L} \operatorname{Res}_{\phi_{p}=0}\left[\prod_{k=2}^{n-2} \zeta_{B_{p}^{(k-1)} C_{p}^{(k-1)}}\left(\phi_{p}\right) \times\right. \\
& \left.\times H^{\check{A}_{p}^{(1)} \check{A}_{p}^{(2)} B_{p}^{(1)}}\left(\phi_{p}\right)\left(\prod_{k=3}^{n-2} H^{C_{p}^{(k-2)} \check{A}_{p}^{(k)} B_{p}^{(k-1)}}\left(\phi_{p}\right)\right) H^{C_{p}^{(n-3)} \check{A}_{p}^{(n-1)} \check{A}_{p}^{(n)}}\left(\phi_{p}\right)\right] \text {. }
\end{aligned}
$$

To prove it, recall that precisely two indices in $\left\{\check{A}_{p}^{(1)}, \check{A}_{p}^{(2)}, \ldots, \check{A}_{p}^{(n)}\right\}$ on l.h.s. are scalars for each $p$. On r.h.s., there are $(3 n-6)$ flavor indices for each $p$

$$
\left\{\check{A}_{p}^{(1)}, \check{A}_{p}^{(2)}, B_{p}^{(1)}\left|C_{p}^{(1)}, \check{A}_{p}^{(3)}, B_{p}^{(2)}\right| \cdots\left|C_{p}^{(n-4)}, \check{A}_{p}^{(n-2)}, B_{p}^{(n-3)}\right| C_{p}^{(n-3)}, \check{A}_{p}^{(n-1)}, \check{A}_{p}^{(n)}\right\}
$$

and the factors $H$ or $\zeta$ are trivial if they consist of the identity fields only. We look for the term of $O\left(\phi_{p}^{-1}\right)$. This comes from the case

( Number of $H$ 's carrying scalars $)-($ Number of $\zeta$ 's carrying scalars $)=1$.

Since $g_{B C}$ is a diagonal metric, this condition is satisfied precisely when two of $\left\{\check{A}_{p}^{(1)}, \check{A}_{p}^{(2)}, \ldots, \check{A}_{p}^{(n)}\right\}$ are scalars. In particular, all the $B$ 's and $C$ 's between a pair of $A$ 's should be scalars,

$$
\check{A}_{p}^{(k)} \neq \mathbf{1} \text { and } \check{A}_{p}^{(\ell)} \neq \mathbf{1} \Rightarrow B_{p}^{(m)} \neq \mathbf{1} \text { and } C_{p}^{(m)} \neq \mathbf{1} \quad(k-1 \leq m \leq \ell-2) .
$$

This completes the proof.

Let us combine the pants decomposition of the color and flavor factors (3.4), (3.8), and substitute them into the $n$-point formula (3.1). The result is

$$
\mathcal{X}\left[\Sigma_{0, n}\right]=\frac{1}{L !} \sum_{\omega, \eta} \delta_{L}\left(\Omega\left[\omega_{1}\right]\left[\omega_{2}\right] \ldots\left[\omega_{n-2}\right]\right) \cdot \prod_{p=1}^{L} \operatorname{Res}_{\phi_{p}=0}\left[\prod_{k=1}^{n-3} \zeta_{B_{p}^{(k)} C_{p}^{(k)}}\left(\phi_{p}\right) \prod_{f=1}^{n-2} \mathcal{X}\left[\mathcal{S}_{f}\right]\right]
$$

where

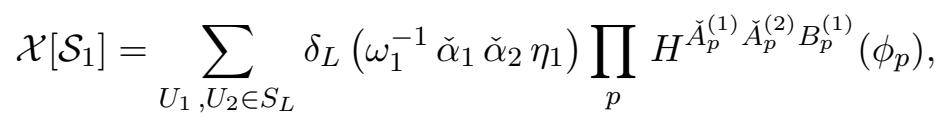

$$
\begin{aligned}
& \mathcal{X}\left[\mathcal{S}_{k}\right]=\sum_{U_{k+1} \in S_{L}} \delta_{L}\left(\omega_{k}^{-1} \eta_{k-1}^{-1} \check{\alpha}_{k+1} \eta_{k}\right) \prod_{p} H^{C_{p}^{(k-1)} \check{A}_{s}^{(k+1)} B_{p}^{(k)}}\left(\phi_{p}\right), \quad(2 \leq k \leq n-3) \\
& \mathcal{X}\left[\mathcal{S}_{n-2}\right]=\sum_{U_{n-1}, U_{n} \in S_{L}} \delta_{L}\left(\omega_{n-2}^{-1} \eta_{n-3}^{-1} \check{\alpha}_{n-1} \check{\alpha}_{n}\right) \prod_{p} H^{C_{p}^{(n-3)} \check{A}_{p}^{(n-1)} \check{A}_{p}^{(n)}}\left(\phi_{p}\right)
\end{aligned}
$$



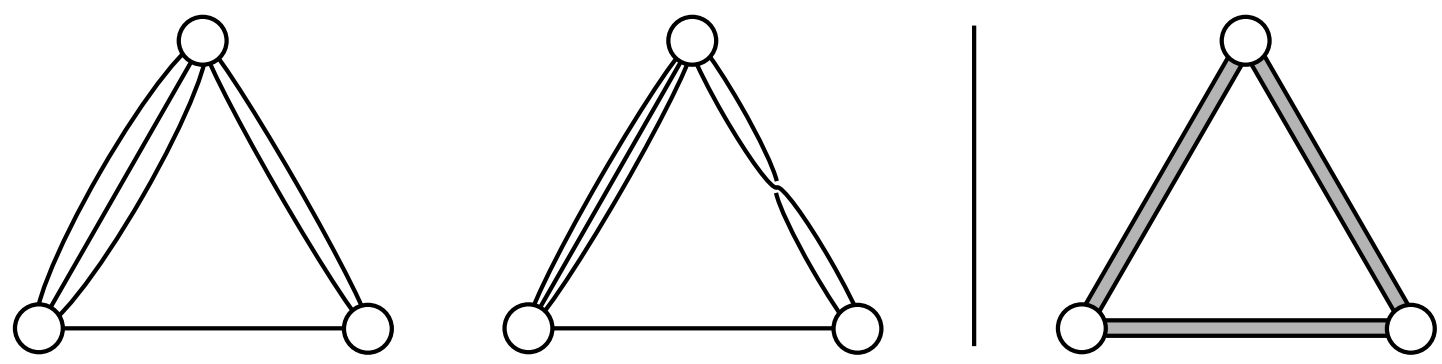

Figure 6. Skeleton reduction of a Feynman graph. Planar Wick-contractions (left) and non-planar consecutive Wick-contractions (center) are reduced to the same skeleton graph (Right).

The equation (3.12) gives the pants decomposition of the $n$-point function addressed in (3.1). The gluing operation consists of the sum over the defect factors $\left\{\omega_{f}\right\}$, the residue operation and inverse metrics $\left\{\zeta_{B C}\right\}$.

Note that the above discussion is different from TFT interpretation discussed in [46]. There we required the invariance of the topological partition function under any cell decompositions of the two-dimensional surface, where each 2-cell is a strip. In other words, the $n$-point functions should decompose into a collection of strips, where each strip collects the local Wick-contractions between $\mathcal{O}_{i}$ and $\mathcal{O}_{j}$. However, it turns out that $G_{n}$ in the $S_{L}$ formalism is compatible with such rewriting only if $n \leq 4$, or equivalently as long as the complete graph $K_{n}$ is planar. For $n \geq 5$, we find obstruction for rewriting, unless we assume certain commutation relation between the permutations for external operators.

\section{Skeleton reduction}

We will perform a skeleton reduction of Feynman graphs.

Let us first clarify what we mean by skeleton reduction. One possible definition of the skeleton reduction is to make a new graph by assembling all edges connecting the same pair of vertices. However, this definition is ambiguous for us, because the Feynman graphs in the double-line notation have the color structure coming from the cyclic ordering of edges around each vertex. We define skeleton as a type of graphs which does not have faces made of two or fewer edges. This definition is convenient because we will eventually relate the skeleton-reduced graph with the complex structure of a Riemann surface. There are a few ways to reduce the original Feynman graphs satisfying this condition. The skeleton reduction used in $[8,9,27]$ assembles the planar, ladder-type internal Wick-contractions, which we call ladder-skeleton. Our skeleton reduction includes non-planar internal Wickcontractions, which simplifies the topology of the reduced graphs. Figure 6 shows a simple example of skeleton reduction.

For simplicity, we consider only the $n$-point functions of single-traces in this section. Our notation is summarized in appendix A.4, and the implementation of the reduction procedure is explained in appendix E. 

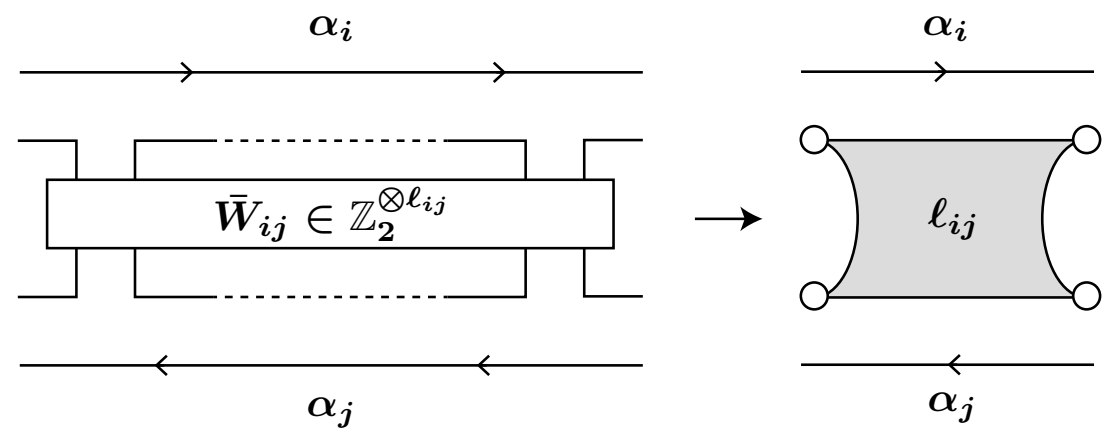

Figure 7. Computing an open two-point function between $\mathcal{O}_{i}$ and $\mathcal{O}_{j}$ by taking the Wickcontractions of consecutive $\ell_{i j}$ fields. White circles represent the color indices at the end-points. Both $\alpha_{i}$ and $\alpha_{j}$ preserve the counterclockwise order.

\subsection{Open two-point functions}

Let us denote a sequence of $\ell$ numbers by

$$
(p+1 \sim p+\ell) \equiv\{p+1, p+2, \ldots, p+\ell\}
$$

and a sequence of consecutive fields by

$$
\left(\Phi^{\boldsymbol{A}_{1 \sim \ell}}\right)_{a_{\ell+1}}^{a_{1}}=\left(\Phi^{A_{1}}\right)_{a_{2}}^{a_{1}}\left(\Phi^{A_{2}}\right)_{a_{3}}^{a_{2}} \ldots\left(\Phi^{A_{\ell}}\right)_{a_{\ell+1}}^{a_{\ell}}
$$

We define open two-point functions by taking all possible Wick-contractions between a pair of sequences,

$$
\begin{aligned}
& \left(\boldsymbol{\Phi}^{\boldsymbol{A}_{p+1 \sim p+\ell}^{(i)}}\right)_{a_{p+\ell+1}^{a}}^{a_{p+1}}\left(\boldsymbol{\Phi}^{\boldsymbol{A}_{q+1 \sim q+\ell^{\prime}}^{(j)}}\right)_{b_{q+\ell^{\prime}+1}}^{b_{q+1}} \\
& =\left.\delta_{\ell, \ell^{\prime}} \sum_{\bar{W}} \prod_{k=1}^{\ell} g^{A_{p+k} B_{q+k^{\prime}}} \delta_{b_{q+k^{\prime}+1}}^{a_{p+k}} \delta_{a_{p+k+1}}^{b_{q+k^{\prime}}}\right|_{q+k^{\prime}=\bar{W}^{-1}(p+k)}
\end{aligned}
$$

where $k^{\prime}=\tau(k)$ for some $\tau \in S_{\ell}$. We sum $\bar{W}$ over the space of "local" Wick-contractions

$$
\bar{W} \in\left\{\prod_{k=1}^{\ell}(p+k, q+\tau(\ell-k)) \mid \tau \in S_{\ell}\right\}, \quad \bar{W} \in \mathbb{Z}_{2}^{\otimes \ell}, \quad \bar{W}^{2}=1 .
$$

We inverted the argument of $\tau$ by $k \mapsto \ell-k$ to keep track of the orientation of the whole graph. We assign the counterclockwise ordering to all external operators. This means that $\alpha_{i}$ and $\alpha_{j}$ rotate the color indices in the opposite directions, as explained graphically in figure 7 .

Let us simplify the color structure of open two-point functions. In (4.3), r.h.s. contains $2 \ell$ color indices, while l.h.s. has the color structure which we express by a matrix $R_{a_{p+\ell+1} b_{q+\ell+1}}^{a_{p+1} b_{q+1}}$. The remaining $2(\ell-1)$ color indices should be contracted. Let us write

$$
\begin{aligned}
\left.\prod_{k=1}^{\ell} \delta_{b_{q+k^{\prime}+1}}^{a_{p+k}} \delta_{a_{p+k+1}}^{b_{q+k^{\prime}}}\right|_{q+k^{\prime}=\bar{W}^{-1}(p+k)} & =\prod_{k=1}^{\ell} \delta_{b_{\alpha_{j}} \bar{W}^{-1}(p+k)}^{a_{p+k}} \delta_{a_{\alpha_{i}(p+k)}}^{b_{\bar{W}^{-1}(p+k)}} \\
& \equiv N_{c}^{Z\left(\alpha_{i} \bar{W} \alpha_{j} \bar{W}^{-1} \mid a_{p+1}, b_{q+1}\right)} R_{a_{p+\ell+1} b_{q+\ell+1}}^{a_{p+1} b_{q+1}}
\end{aligned}
$$


Here $Z(\tilde{\omega} \mid a, b)$ counts the number of cycles in $\tilde{\omega}$ without $a$ or $b$. To understand $Z(\tilde{\omega} \mid a, b)$, recall that the permutation $\tilde{\omega}=\alpha_{i} \bar{W} \alpha_{j} \bar{W}^{-1}$ represents the faces of the part of the original Feynman graph. ${ }^{21}$ Since all Wick-contractions are locally taken between $\mathcal{O}_{i}$ and $\mathcal{O}_{j}$, the repeated applications of $\omega$ send the upper indices $\left(a_{p+1}, b_{q+1}\right)$ to the lower indices $\left(a_{p+\ell+1}, b_{q+\ell+1}\right)$. In other words, we cannot go outside the interval between $\left(a_{p+1}, b_{q+1}\right)$ and $\left(a_{p+\ell+1}, b_{q+\ell+1}\right)$, because all edges are consecutive. If we write

$$
\begin{aligned}
\tilde{\omega}= & \left(a_{p+1}, \omega\left(a_{p+1}\right), \omega^{2}\left(a_{p+1}\right), \ldots, c, \ldots\right) \times \\
& \times\left(b_{q+1}, \omega\left(b_{q+1}\right), \omega^{2}\left(b_{q+1}\right), \ldots, c^{\prime}, \ldots\right) \times(\text { the other internal cycles })
\end{aligned}
$$

where $\left(c, c^{\prime}\right)$ is equal to $\left(a_{p+\ell+1}, b_{q+\ell+1}\right)$ or $\left(b_{q+\ell+1}, a_{p+\ell+1}\right)$, we find

$$
Z\left(\tilde{\omega} \mid a_{p+1}, b_{q+1}\right) \equiv \text { (the number of the other internal cycles). }
$$

By substituting (4.5) into (4.3), we obtain

$$
\begin{aligned}
& \left(\boldsymbol{\Phi}^{\boldsymbol{A}_{p+1 \sim p+\ell}}\right)_{a_{p+\ell+1}}^{a_{p+1}}\left(\boldsymbol{\Phi}^{\boldsymbol{B}_{q+1 \sim q+\ell^{\prime}}}\right)_{b_{q+\ell^{\prime}+1}^{b_{q+1}}} \\
& =\delta_{\ell, \ell^{\prime}} \sum_{\bar{W}}\left(\prod_{k=1}^{\ell} g^{A_{p+k} B_{\bar{W}-1}(p+k)}\right) N_{c}^{Z\left(\alpha_{i} \bar{W} \alpha_{j} \bar{W}^{-1} \mid a_{p+1}, b_{q+1}\right)} R_{a_{p+\ell+1} b_{q+\ell+1}}^{a_{p+1} b_{q+1}} .
\end{aligned}
$$

Powers of $\boldsymbol{N}_{\boldsymbol{c}}$ and the color $\boldsymbol{R}$-matrix. As a linear operator, the color matrix $R_{a^{\prime} b^{\prime}}^{a b}$ is just a product of two $\delta$-functions. Its index structure is correlated with the powers of $N_{c}$ as

$$
N_{c}^{Z\left(\alpha_{i} \bar{W} \alpha_{j} \bar{W}^{-1} \mid a_{p+1}, b_{q+1}\right)} R_{a_{p+\ell+1} b_{q+\ell+1}}^{a_{p+1} b_{q+1}}=\left\{\begin{array}{l}
N_{c}^{\ell-2 n+1} \delta_{b_{q+\ell+1}}^{a_{p+1}} \delta_{a_{p+\ell+1}}^{b_{q+1}} \\
N_{c}^{\ell-2 n} \delta_{a_{p+\ell+1}}^{a_{p+1}} \delta_{b_{q+\ell+1}}^{b_{q+1}}
\end{array} \quad\left(\text { for some } n \in \mathbb{Z}_{\geq 1}\right) .\right.
$$

We can prove this selection rule as follows. If we multiply $\delta_{a_{p+1}}^{a_{p+\ell+1}} \delta_{b_{q+1}}^{b_{q+\ell+1}}$, the equation (4.8) becomes part of the two-point function of single-traces, written as

$$
\delta_{a_{p+1}}^{a_{p+\ell+1}} \delta_{b_{q+1}}^{b_{q+\ell+1}} \prod_{k=1}^{\ell} \delta_{b_{\alpha_{j}} \bar{W}^{-1}(p+k)}^{a_{p+k}} \delta_{a_{\alpha_{i}(p+k)}}^{b_{\bar{W}-1}(p+k)}=N_{c}^{C\left(\alpha_{i}^{\prime} \bar{W} \alpha_{j}^{\prime} \bar{W}^{-1}\right)}, \quad\left(\alpha_{i}^{\prime}, \alpha_{j}^{\prime} \in \mathbb{Z}_{\ell+1}\right) .
$$

Since $\alpha_{i}^{\prime}$ and $\alpha_{j}^{\prime}$ have the same cycle type and belong to the same conjugacy class, we may redefine $\bar{W}$ to rewrite

$$
\alpha_{i}^{\prime} \bar{W} \alpha_{j}^{\prime} \bar{W}^{-1}=\alpha_{i}^{\prime} \bar{W}^{\prime} \alpha_{i}^{\prime-1} \bar{W}^{\prime-1} .
$$

This is an element of the commutator subgroup of $S_{\ell+1}$, namely $A_{\ell+1}$, consisting of an even number of transpositions. Thus the power of $N_{c}$ changes only by an even number when $\bar{W}^{\prime}$ changes, which translates into (4.8).

The powers of $N_{c}$ are also determined by $\tau \in S_{\ell}$ in (4.4), and we can rewrite

$$
N_{c}^{\bar{Z}(\tau)} \equiv N_{c}^{Z\left(\alpha_{i} \bar{W} \alpha_{j} \bar{W}^{-1} \mid a_{p+1}, b_{q+1}\right)} .
$$

\footnotetext{
${ }^{21}$ Since $\bar{W}$ is a set of local Wick-contractions as in (4.4), $\tilde{\omega}$ knows only the faces inside $\mathcal{O}_{i}$ and $\mathcal{O}_{j}$.
} 


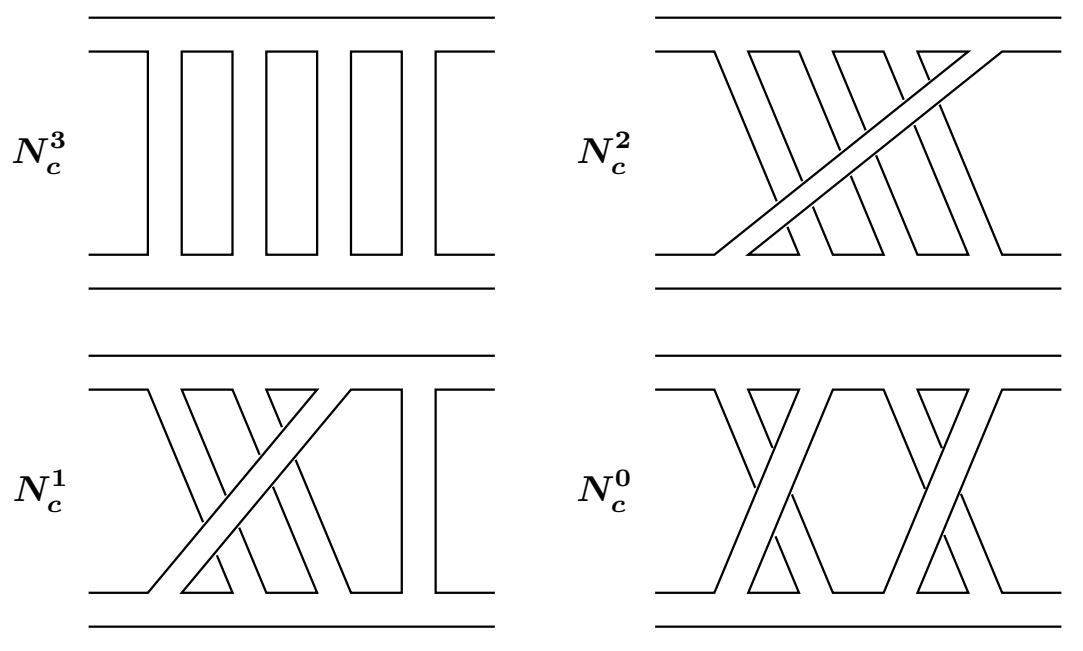

Figure 8. Open two-points at $\ell=4$. We find $\tau=(1)(2)(3)(4)$ and (1234) (top left and right), $(123)(4)$ and $(12)(34)$ (bottom left and right); $R_{a^{\prime} b^{\prime}}^{a b}=\delta_{b^{\prime}}^{a} \delta_{a^{\prime}}^{b}$ and $\delta_{a^{\prime}}^{a} \delta_{b^{\prime}}^{b}$ for left and right figures.

Some examples are shown in figure 8. $\bar{Z}$ is maximal if $\tau$ is identity,

$$
\tau=\mathbf{1} \in S_{\ell} \quad \Leftrightarrow \quad \bar{Z}(\tau)=\ell-1
$$

which corresponds to the planar case. The $O\left(1 / N_{c}\right)$ terms come from cyclic permutations,

$$
\tau \in \mathbb{Z}_{\ell}, \tau \neq \mathbf{1} \quad \Leftrightarrow \quad \bar{Z}(\tau)=\ell-2
$$

The $O\left(1 / N_{c}^{2}\right)$ terms come from cyclic permutations of lower order with consecutive numbers,

$$
\tau=(s, s+1, \ldots, s+k) \in \mathbb{Z}_{k}(k<\ell), \tau \neq \mathbf{1} \quad \Leftrightarrow \quad \bar{Z}(\tau)=\ell-3 .
$$

\subsection{Relabeling Wick-contractions}

The skeleton reduction reorganizes the structure of Wick-contractions. This structure can be explained in two ways, by using $W_{i j, \rho}$ or equivalently $\left(V_{i j, \rho}, \tau_{i j, \rho}\right)$.

In the first explanation, we introduce $\left\{\ell_{i j}^{(\rho)}\right\}$, where $\rho=1,2, \ldots, r_{i j}$ labels nonconsecutive sets of Wick-edges between $\mathcal{O}_{i}$ and $\mathcal{O}_{j}$. In other words, $r_{i j}$ counts the number of open two-point functions between $\mathcal{O}_{i}$ and $\mathcal{O}_{j}$. They satisfy

$$
\ell_{i i}^{(\rho)}=0, \quad \ell_{i j}^{(\rho)}=\ell_{j i}^{(\rho)}, \quad \ell_{i j}=\sum_{\rho=1}^{r_{i j}} \ell_{i j}^{(\rho)}, \quad \sum_{i<j}^{n} \sum_{\rho=1}^{r_{i j}} \ell_{i j}^{(\rho)}=L
$$

which generalizes (1.3). Note that planar graphs may have $r_{i j}>1$. In this notation, $G_{n}$ becomes

$$
G_{n}=\sum_{\left\{\ell_{i j}^{(\rho)}\right\}} \prod_{i<j}^{n} \prod_{\rho=1}^{r_{i j}} \sum_{W_{i j, \rho}} \prod_{p=1}^{\ell_{i j}^{(\rho)}}\left[g^{A_{p}^{(i)} A_{q}^{(j)}} \delta_{b_{\alpha_{j}(q)}}^{a_{p}} \delta_{a_{\alpha_{i}(p)}}^{b_{q}}\right]_{q=W_{i j, \rho}^{-1}(p)}
$$


where $\left\{W_{i j, \rho}\right\}$ is completely equivalent to the original $W$,

$$
\prod_{i<j}^{n} \prod_{\rho=1}^{r_{i j}} W_{i j, \rho}=W \in \mathbb{Z}_{2}^{\otimes L} \subset S_{2 L}
$$

Each $W_{i j, \rho}$ takes the form

$$
W_{i j, \rho}=\prod_{k=1}^{\ell_{i j}^{(\rho)}}\left(p+k, q+\tau_{i j, \rho}\left(\ell_{i j}^{(\rho)}-k\right)\right)
$$

as in (4.4).

The second explanation was given in Introduction. We split $\mathcal{O}_{i}$ into the sequential fields $\left\{\boldsymbol{\Phi}_{r}^{(i)}\right\}$ in (4.2), and call it a partition of $\mathcal{O}_{i}$,

$$
\mathcal{O}_{i}=\operatorname{tr}_{L_{i}}\left(\alpha_{i} \Phi^{A_{1}^{(i)}} \Phi^{A_{2}^{(i)}} \ldots \Phi^{A_{L}^{(i)}}\right) \quad \stackrel{\text { partition }}{\longrightarrow} \overline{\mathcal{O}}_{i}=\operatorname{tr}_{\bar{L}_{i}}\left(\bar{\alpha}_{i} \Phi_{1}^{(i)} \Phi_{2}^{(i)} \ldots \Phi_{\bar{L}_{i}}^{(i)}\right) .
$$

We take open two-point functions as the internal Wick-contractions between $\boldsymbol{\Phi}_{p}^{(i)}$ and $\boldsymbol{\Phi}_{q}^{(j)}$. Now the $n$-point functions contain three sums.

i) Sum over the partitions $\mathcal{O}_{i}$ into $\left\{\overline{\mathcal{O}}_{i}\right\}$.

ii) Sum over the external Wick-contractions, for all possible pairings $\{(i, p),(j, q)\}$ of the sequential fields $\left\{\boldsymbol{\Phi}_{p}^{(i)}, \boldsymbol{\Phi}_{q}^{(j)}\right\}$.

iii) Sum over the internal Wick-contractions, for each $\left\langle\boldsymbol{\Phi}_{p}^{(i)} \boldsymbol{\Phi}_{q}^{(j)}\right\rangle$ as in (4.7). The choice of $\tau_{i j, \rho}$ in (4.18) is equivalent to the internal Wick-contraction.

For a given partition $\left\{\overline{\mathcal{O}}_{i}\right\}$, the equivalence of the two explanations can be understood as follows. We relabel the color indices of $\mathcal{O}_{i}$ as

$$
p \in\left\{1,2, \ldots, L_{i}\right\}=\left\{(\mathrm{p}, \bar{p}) \mid \bar{p} \in\left\{1,2, \ldots, \ell_{\mathrm{p}}\right\}, \mathrm{p} \in\left\{1,2, \ldots, \bar{L}_{i}\right\}\right\}
$$

where $\mathrm{p}$ labels the open end-points and $\bar{p}$ labels the internal indices. The equation (4.19) can be written as

$$
\mathcal{O}_{i}=\prod_{p=1}^{L_{i}}\left(\Phi^{A_{p}}\right)_{a_{\alpha_{i}(p)}}^{a_{p}} \stackrel{\text { partition }}{\longrightarrow} \quad \overline{\mathcal{O}}_{i}=\prod_{\mathrm{p}=1}^{\bar{L}_{i}}\left(\boldsymbol{\Phi}_{p}^{(i)}\right)_{a_{\bar{\alpha}_{i}(\mathrm{p})}}^{a_{\mathrm{p}}}=\prod_{\mathrm{p}=1}^{\bar{L}_{i}} \prod_{\bar{p}=1}^{\ell_{\mathrm{p}}}\left(\Phi^{A_{\mathrm{p}, \bar{p}}}\right)_{a_{\mathrm{p}+\bar{p}}}^{a_{\mathrm{p}+\bar{p}-1}} .
$$

Here the reduced color permutation $\bar{\alpha}_{i} \in S_{\bar{L}_{i}}$ shifts the open end-points $\{\mathrm{p}\}$, and thus $a_{\bar{\alpha}_{i}(\mathrm{p})}=a_{\mathrm{p}+\ell_{\mathrm{p}}}$. We write the Wick-contractions in the new notation as

$$
W \ni\left(a_{p}, b_{q}\right)=\left(a_{\mathbf{p}, \bar{p}}, b_{\mathbf{q}, \bar{q}}\right) \quad \leftrightarrow \quad\left(a_{\mathbf{p}}, b_{\mathbf{q}}\right) \times(\bar{p}, \bar{q}) \in(V, \tau) .
$$

The new permutation $V$ is equivalent to the external Wick-contraction. The relation between $W_{i j, \rho}$ and $\left(V_{i j, \rho}, \tau_{i j, \rho}\right)$ is also depicted in figure 9 .

The open two-point function (4.7) can be rewritten as

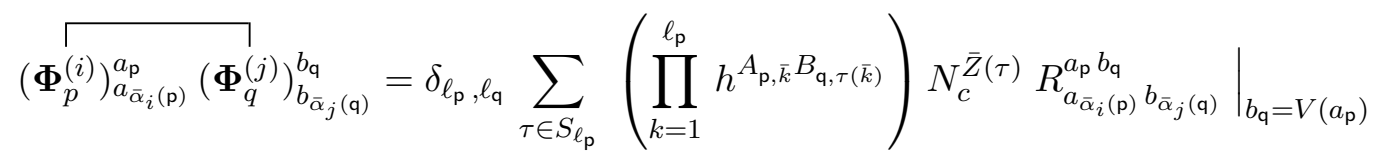



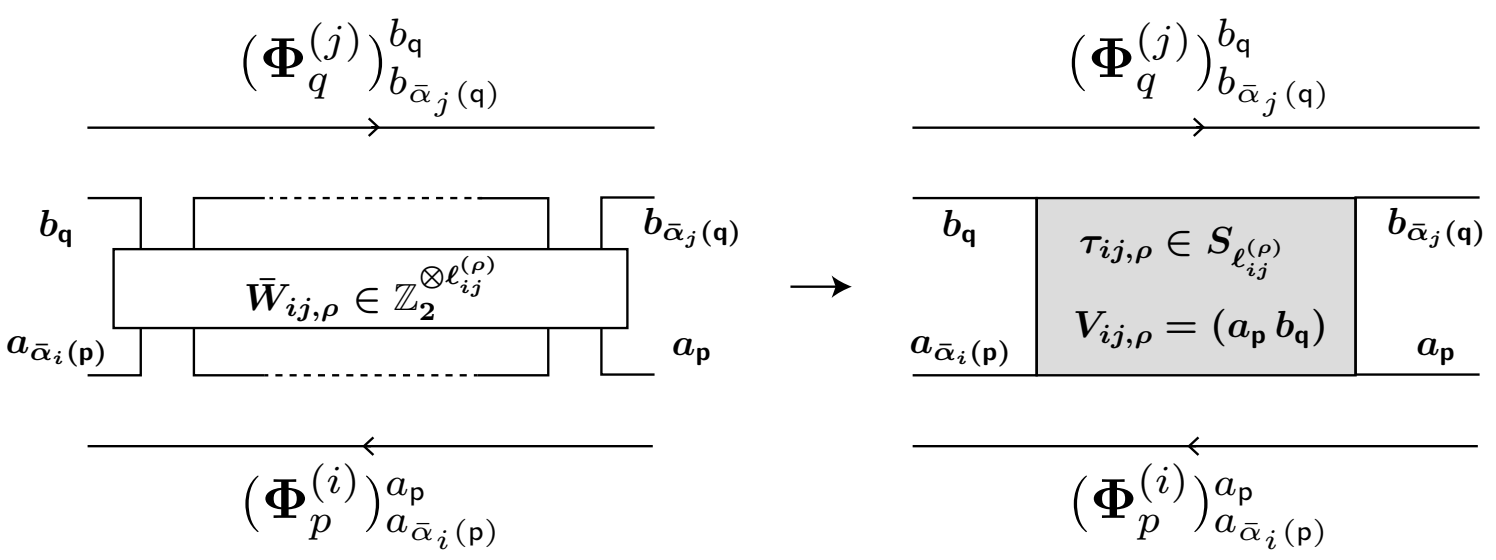

Figure 9. Rewriting the open two-point function in figure 7 in terms of $(V, \tau)$. The color indices at the four end-points are denoted by $\left(a_{\mathbf{p}}, b_{\mathbf{q}}, a_{\bar{\alpha}_{i}(\mathbf{p})}, b_{\bar{\alpha}_{j}(\mathbf{q})}\right)$.

where $\bar{Z}(\tau)$ is given in (4.11), and we replaced $g^{A B}$ by $h^{A B}$ in (2.16) for a later purpose. In this notation, $G_{n}$ is given by

$$
G_{n}=\sum_{\left\{\text {partition of } \mathcal{O}_{i}\right\}} \sum_{V: \text { external }} \sum_{\tau: \text { internal }} \prod_{i<j}^{n} \prod_{\rho=1}^{r_{i j}}\left[\left(\prod_{k=1}^{\ell_{i j}^{(\rho)}} h^{A_{\mathrm{p}, \overline{\mathrm{q}}}^{(i)} A_{\mathrm{q}, \tau(\bar{k})}^{(j)}}\right) N_{c}^{\bar{Z}\left(\tau_{i j, \rho}\right)} R_{a_{a_{\bar{\alpha}_{i}(\mathrm{p})}^{(i)}}^{a_{\mathrm{p}}^{(i)} a_{\mathrm{q}}^{(j)}}}^{(j)}\right]
$$

where

$$
\mathbb{Z}_{2}^{\otimes \bar{L}} \ni V=\prod_{i<j}^{n} \prod_{\rho} V_{i j, \rho}=\prod_{i<j}^{n} \prod_{\rho}\left(a_{\mathrm{p}}^{(i)} a_{\mathrm{q}}^{(j)}\right), \quad \tau=\prod_{i<j}^{n} \prod_{\rho} \tau_{i j, \rho} .
$$

In appendix C, we simplify (4.24) including the range of summations.

\subsection{Face-based skeleton formula}

We discuss how to reconstruct the $n$-point function of single-trace operators from the graph data, following our discussion in section 2.3. In (C.27) we fix $\bar{\alpha}$ and take a sum over $V$. Below we fix $V$ and sum over the face permutation $\nu$ of a skeleton graph. We also need the set of internal Wick-contractions $\tau$ to recover the full $n$-point function.

\subsubsection{Sum over the skeleton faces}

To begin with, let us explain how to sum over the faces of skeleton graphs. We choose a partition

$$
\ell=\left(\ell_{1}, \ell_{2}, \ldots, \ell_{\bar{L}}\right) \vdash L .
$$

We need $\bar{L} \geq n / 2$ for general $n$-point functions, and $\bar{L} \geq n-1$ for connected $n$-point. We impose the ordering

$$
\ell_{1} \geq \ell_{2} \geq \cdots \geq \ell_{\bar{L}}>0
$$

Since the ordering of $\left\{\boldsymbol{\ell}_{i}\right\}$ in (4.26) is unimportant, we use $\boldsymbol{\ell}$ and [ $\left.\boldsymbol{\ell}\right]$ interchangeably. Let us denote the $p$-th edge of length $\ell$ by $p_{\ell}$, and use $\left\{1_{\ell_{1}}, 1_{\ell_{1}}^{\prime}, \ldots, \bar{L}_{\ell_{\bar{L}}}, \bar{L}_{\ell_{\bar{L}}}^{\prime}\right\}$ to label the edges 

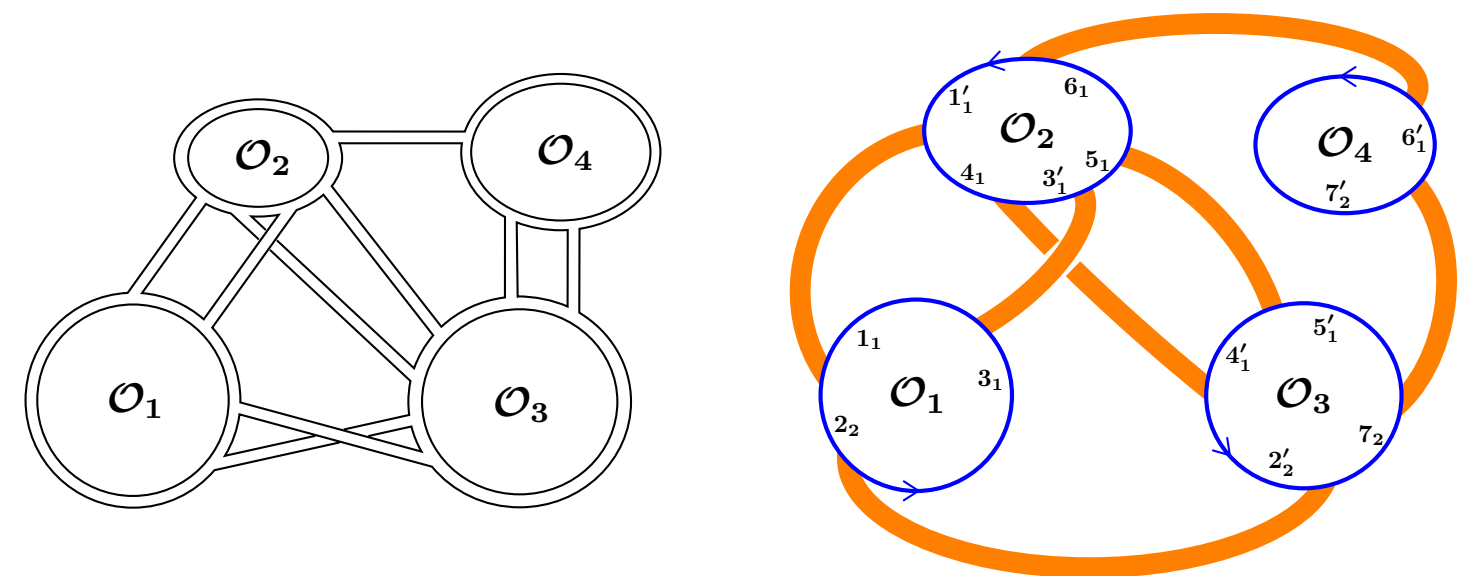

Figure 10. Skeleton reduction of a set of the Wick-contractions in $\left\langle\mathcal{O}_{1} \mathcal{O}_{2} \mathcal{O}_{3} \mathcal{O}_{4}\right\rangle$ with $\left(L_{1}, L_{2}, L_{3}, L_{4}\right)=(4,5,6,3)$. These graphs are the same as in figure 21 .

of a skeleton graph. We set

$$
V_{\bullet} \equiv \prod_{\bar{p}=1}^{\bar{L}}\left(p_{\ell_{p}} p_{\ell_{p}}^{\prime}\right) .
$$

We denote by $\boldsymbol{r}_{k}$ the number of consecutive Wick-contractions with $k$ fields as in (C.20). Since there are $\boldsymbol{r}_{k}$ skeleton edges with length $k$, the symmetry of relabeling is

$$
\text { Aut } V=\text { Aut } V(\ell) \equiv \bigotimes_{k} S_{\boldsymbol{r}_{k}}\left[\mathbb{Z}_{2}\right] \subset S_{\bar{L}}\left[\mathbb{Z}_{2}\right]
$$

There is a bijection between the set of unlabeled ladder-skeleton graphs and face permutations generalizing $(2.49),{ }^{22}$

\{Ladder-skeleton graphs with $2 \bar{L}$ unlabeled vertices $\} \quad \leftrightarrow \quad \nu \in S_{2 \bar{L}}^{\times \times} /$Aut $V$.

Here we defined the group $S_{2 \bar{L}}^{\times \times} \subset S_{2 \bar{L}}$ without one- or two-cycles,

$$
\nu \in S_{2 \bar{L}}^{\times \times} \quad \Leftrightarrow \quad p_{\ell} \neq \nu\left(p_{\ell}\right) \neq \nu^{2}\left(p_{\ell}\right) \quad\left(\forall p_{\ell}\right) .
$$

In r.h.s. of (4.30), $\nu$ permutes $\left\{1_{\ell_{1}}, 1_{\ell_{1}}^{\prime}, \ldots, \bar{L}_{\ell_{\bar{L}}}, \bar{L}_{\ell_{\bar{L}}}^{\prime}\right\}$, where $p_{\ell}$ means the $p$-th edge of length $\ell$. Due to the redundancy of relabeling, $\nu$ is in one-to-one correspondence with the coset $S_{2 \bar{L}}^{\times \times} /$Aut $V$. The correspondence (4.30) can be proven as in section 2.3. Following appendix C.3.3, we define the space $\left(S_{2 \bar{L}}^{\times \times}\right)_{\text {phys }}$ as

$$
\{\text { Skeleton graphs with } 2 \bar{L} \text { unlabeled vertices }\} \quad \leftrightarrow \quad \nu \in\left(S_{2 \bar{L}}^{\times \times} / \operatorname{Aut} V\right)_{\text {phys }}
$$

where we exclude the mutually adjacent pairs in r.h.s. . Unfortunately, we do not find a simple algebraic characterization of $\left(S_{2 \bar{L}}^{\times \times} / \text {Aut } V\right)_{\text {phys }}$.

\footnotetext{
${ }^{22}$ The ladder-skeleton graph is made by assembling the planar, ladder-type consecutive Wick-contractions.
} 


\subsubsection{Reconstruction of correlators}

Let us explain how to reconstruct correlators from a skeleton graph. We set $\bar{\alpha}_{\bullet} \equiv \nu V_{\bullet} \in S_{2 \bar{L}}$ and use $\bar{\alpha}_{\bullet}$ to make all skeleton-rims oriented. We denote the cycle decomposition of $\bar{\alpha}_{\bullet}$ by

$$
\bar{\alpha}_{\bullet}=\left(\bar{\alpha}_{1}, \bar{\alpha}_{2}, \ldots, \bar{\alpha}_{n}\right), \quad\left(\bar{\alpha}_{i} \in \mathbb{Z}_{\bar{L}_{i}}\right) .
$$

Parameterize each cycle by using skeleton edges as $\bar{\alpha}_{i}=\left(p^{(i, 1)} p^{(i, 2)} \ldots p^{\left(i, \bar{L}_{i}\right)}\right)$, where $p^{(i, k)}$ is a shorthand of $p_{\ell_{p(i, k)}}$ or $p_{\ell_{p(i, k)}}^{\prime}$ introduced in (4.28). Define

$$
\Delta\left(\bar{\alpha}_{i}\right)=\sum_{k=1}^{\bar{L}_{i}} \ell_{p(i, k)}
$$

which should count the number of scalars in $\mathcal{O}_{i}$. We impose the conditions that graph data should match those of physical data,

$$
n=n_{\mathrm{ex}}, \quad \Delta\left(\bar{\alpha}_{i}\right)=L_{\mathrm{ex}, i} \quad \text { for } \quad\left\langle\mathcal{O}_{1} \mathcal{O}_{2} \ldots \mathcal{O}_{n_{\mathrm{ex}}}\right\rangle, \quad\left(\alpha_{i} \in S_{L_{\mathrm{ex}, i}}\right) .
$$

These conditions determine the cycle type of $\alpha=\prod_{i} \alpha_{i} \in S_{2 L}$. Thus, the equations (4.35) rewrite the constraint $\delta_{\text {cycle-type }}\left(\alpha_{\text {ex }}^{-1}, \omega W_{\bullet}\right)$ used in (2.65). Figure 10 shows an example of the skeleton reduction using the notation introduced here.

Next, we generate a set of ordered lists from the permutation $\alpha_{\bullet}$ like (2.51). In (2.52) we defined the map CL as the wreath product, namely the composition of the cyclic translations and the permutations of the cycles of the same length. Here we consider the cyclic translations and permutations separately.

Let us define the map from a permutation to a set of the ordered reduced lists, denoted by $\mathbf{C R}$. This consists of the permutations of the operators with the same dimensions $\Delta\left(\bar{\alpha}_{i}\right)=\Delta\left(\bar{\alpha}_{j}\right)$. For example, when $\bar{\alpha}_{\bullet}=\left(1_{2} 2_{1}\right)\left(1_{2}^{\prime} 3_{1}^{\prime}\right)\left(2_{1}^{\prime} 3_{1}^{\prime}\right)$ we get

$$
\mathbf{C R}\left(\bar{\alpha}_{\bullet}\right)=\left\{\left(1_{2}, 2_{1}\right)\left(1_{2}^{\prime}, 3_{1}^{\prime}\right)\left(2_{1}^{\prime}, 3_{1}^{\prime}\right),\left(1_{2}^{\prime}, 3_{1}^{\prime}\right)\left(1_{2}, 2_{1}\right)\left(2_{1}^{\prime}, 3_{1}^{\prime}\right)\right\}
$$

because $\bar{\alpha}_{1}$ and $\bar{\alpha}_{2}$ having $\Delta\left(\bar{\alpha}_{1}\right)=\Delta\left(\bar{\alpha}_{2}\right)=3$ can be interchanged. The map $\mathbf{C R}$ depends only on the operator dimensions $\Delta\left(\bar{\alpha}_{i}\right)=\Delta\left(\bar{\alpha}_{j}\right)$, and they may have $\bar{L}_{i} \neq \bar{L}_{j}$. If we denote the cycle type of $\alpha$ by $\lambda=\left[1^{\lambda_{1}} 2^{\lambda_{2}} \ldots\right] \vdash 2 L$, then

$$
|\mathbf{C R}(\lambda)|=\left|\prod_{k} S_{\lambda_{k}}\right|=\prod_{k} \lambda_{k} !
$$

The cyclic translations will be discussed later.

Choose an element $\bar{\beta}=\left(\bar{\beta}_{1}, \ldots, \bar{\beta}_{n}\right)$ from $\mathbf{C R}\left(\bar{\alpha}_{\bullet}\right)$, and use it to relate the rim labels to the flavor-like indices

$$
\bar{\beta}_{i}:\left\{1_{\ell_{1}}, 1_{\ell_{1}}^{\prime}, \ldots, \bar{L}_{\ell_{\bar{L}}}, \bar{L}_{\ell_{\bar{L}}}^{\prime}\right\} \rightarrow\left\{0,1,2, \ldots, \bar{L}_{i}\right\}
$$

such that $\bar{\beta}_{i}^{-1}(x)$ is uniquely defined if and only if $x \neq 0$. We parameterize $\bar{\alpha}$ as

$$
\bar{\alpha}=\left(\begin{array}{c}
\bar{\alpha}_{1} \\
\bar{\alpha}_{2} \\
\vdots \\
\bar{\alpha}_{n}
\end{array}\right)=\left(\begin{array}{ccc}
\left(\bar{\beta}_{1}^{-1}(1)\right. & \ldots & \left.\bar{\beta}_{1}^{-1}\left(\bar{L}_{1}\right)\right) \\
\left(\bar{\beta}_{2}^{-1}(1)\right. & \ldots & \left.\bar{\beta}_{2}^{-1}\left(\bar{L}_{2}\right)\right) \\
\vdots & \\
\left(\bar{\beta}_{n}^{-1}(1)\right. & \ldots & \left.\bar{\beta}_{n}^{-1}\left(\bar{L}_{n}\right)\right)
\end{array}\right) .
$$


We can better understand this notation by introducing the extended reduced operator

$$
\hat{\mathcal{O}}_{i}=\operatorname{tr}\left(\bar{\alpha}_{i} \boldsymbol{\Phi}_{1}^{(i)} \boldsymbol{\Phi}_{2}^{(i)} \ldots \boldsymbol{\Phi}_{\bar{L}_{i}}^{(i)}\right) \times \operatorname{tr}(\mathbf{1})^{\bar{L}-\bar{L}_{i}}
$$

where the length of the sequential fields $\boldsymbol{\Phi}_{r}^{(i)}$ is found from $\bar{\beta}_{i}^{-1}(r) \in\left\{1_{\ell_{1}}, 1_{\ell_{1}}^{\prime}, \ldots, \bar{L}_{\ell_{\bar{L}}}, \bar{L}_{\ell_{\bar{L}}}^{\prime}\right\}$ for $r \neq 0$. The total number of scalar fields in $\hat{\overline{\mathcal{O}}}_{i}$ is given by

$$
L_{i}=\sum_{E \text { from }\left\{\bar{\beta}_{i}^{-1}(1), \ldots, \bar{\beta}_{i}^{-1}\left(\bar{L}_{i}\right)\right\}} \ell_{E} .
$$

We construct the reduced Wick-contraction matrix by

$$
\bar{\mu}_{\bar{\beta}}=\left(\begin{array}{ccccc}
\bar{\mu}_{1,1} & \bar{\mu}_{1,1^{\prime}} & \ldots & \bar{\mu}_{1, \bar{L}} & \bar{\mu}_{1, \bar{L}^{\prime}} \\
\bar{\mu}_{2,1} & \bar{\mu}_{2,1^{\prime}} & \ldots & \bar{\mu}_{2, \bar{L}} & \bar{\mu}_{2, \bar{L}^{\prime}} \\
\vdots & & & & \\
\bar{\mu}_{n, 1} & \bar{\mu}_{n, 1^{\prime}} & \ldots & \bar{\mu}_{n, \bar{L}} & \bar{\mu}_{n, \bar{L}^{\prime}}
\end{array}\right), \quad \bar{\mu}_{i, s}= \begin{cases}\boldsymbol{A}_{\bar{\beta}_{i}(s)}^{(i)} & \left(\bar{\beta}_{i}(s) \neq 0\right) \\
\mathbf{1} & \left(\bar{\beta}_{i}(s)=0\right) .\end{cases}
$$

The reduced Wick-contraction matrix $\bar{\mu}_{\bar{\beta}}$ is in one-to-one correspondence with the external Wick-contraction of the reduced operators. For example, we find

$$
\begin{aligned}
&\left\langle\operatorname{tr}\left(\boldsymbol{\Phi}_{1}^{(1)} \boldsymbol{\Phi}_{2}^{(1)}\right) \operatorname{tr}\left(\mathbf{\Phi}_{1}^{(2)} \boldsymbol{\Phi}_{2}^{(2)}\right) \operatorname{tr}\left(\boldsymbol{\Phi}_{1}^{(3)} \boldsymbol{\Phi}_{2}^{(3)}\right)\right\rangle \leftrightarrow \\
& \leftrightarrow\left(\begin{array}{cccc}
\boldsymbol{A}_{1 \sim \ell_{1 \mid 1}} & \boldsymbol{A}_{\boldsymbol{\ell}_{1 \mid 1}+1 \sim L_{1}} & & \\
& \boldsymbol{B}_{1 \sim \ell_{2 \mid 1}} & \boldsymbol{B}_{\ell_{2 \mid 1}+1 \sim L_{2}} & \\
& & \boldsymbol{C}_{1 \sim \ell_{3 \mid 1}} & \boldsymbol{C}_{\ell_{3 \mid 1}+1 \sim L_{3}}
\end{array}\right)
\end{aligned}
$$

We introduce the flavor factor for the $E$-th edge by

$$
h\left(\boldsymbol{\tau} \mid \boldsymbol{A}_{\mathrm{p}}^{(i)}, \boldsymbol{A}_{\mathrm{q}}^{(j)}\right)=\prod_{\bar{k}=1}^{\ell_{E}} h^{\boldsymbol{A}_{\mathrm{p}, \bar{k}}^{(i)} \boldsymbol{A}_{\mathrm{q}, \tau_{E}(\bar{k})}^{(j)}}
$$

which agrees with the flavor factor in (4.23). We generalize $h$ as

$$
h\left(\boldsymbol{\tau} \mid \boldsymbol{A}_{1} \boldsymbol{A}_{1^{\prime}} \ldots \boldsymbol{A}_{n} \boldsymbol{A}_{n^{\prime}}\right)=\frac{1}{2(2 n-2) !} \sum_{\pi \in S_{2 n}} h\left(\boldsymbol{\tau} \mid \boldsymbol{A}_{\pi(1)}, \boldsymbol{A}_{\pi\left(1^{\prime}\right)}\right) \prod_{j=2}^{n} \delta_{\mathbf{1}}^{\boldsymbol{A}_{(\pi(j))}} \delta_{\mathbf{1}}^{\boldsymbol{A}_{\left(\pi\left(j^{\prime}\right)\right)}} .
$$

like (2.61). We define the flavor function of $\bar{\mu}_{\bar{\beta}}$ by

$$
\mathbf{F}\left(\boldsymbol{\tau} \mid \bar{\mu}_{\bar{\beta}}\right)=\prod_{s=1}^{\bar{L}} h\left(\boldsymbol{\tau} \mid \bar{\mu}_{1, s} \bar{\mu}_{1, s^{\prime}} \bar{\mu}_{2, s} \bar{\mu}_{2, s^{\prime}} \ldots \bar{\mu}_{n, s} \bar{\mu}_{n, s^{\prime}}\right)
$$

where r.h.s. is a generalization of (2.22). 
Now we consider the cyclic translations $\mathbb{Z}_{\alpha}=\prod_{i} \mathbb{Z}_{L_{i}}$. In fact, although the formula (4.45) computes the flavor factor for a set of reduced operators $\overline{\mathcal{O}}_{i}$, we did not specify the relation between $\overline{\mathcal{O}}_{i}$ and $\mathcal{O}_{i}$ Let us rewrite $h\left(\boldsymbol{\tau} \mid \boldsymbol{A}_{r}^{(i)}, \boldsymbol{A}_{s}^{(j)}\right)$ in (4.43) as

$$
\left.h\left(\boldsymbol{\tau} \mid \boldsymbol{A}_{\mathrm{p}}^{(i)}, \boldsymbol{A}_{\mathbf{q}}^{(j)}\right)=\prod_{k=1}^{\ell_{E}} h^{\boldsymbol{A}_{p_{0}+k}^{(i)} \boldsymbol{A}_{q_{0}+\tau_{E}}^{\left(\ell_{E}-k\right)}}, \quad \text { (for some } p_{0}, q_{0}\right)
$$

using (4.4). The flavor index $p_{0} \in\left\{1,2, \ldots, L_{i}\right\}$ determines the relation between the reduced operator $\overline{\mathcal{O}}_{i}$ in (4.21) and the original operator $\mathcal{O}_{i}$ in (2.1). The flavor factors with different $p_{0}$ are related by the cyclic translations $\mathbb{Z}_{\alpha}$

$$
\mathbb{Z}_{\alpha} \ni \boldsymbol{z}=\left(z_{1}, z_{2}, \ldots, z_{n}\right), \quad z_{i}: p_{0} \mapsto p_{0}+1 \quad\left(\bmod L_{i}\right) .
$$

This symmetry was also part of the stabilizer (2.54). We write

$$
\mathbf{F}\left(\boldsymbol{\tau} \mid \boldsymbol{z} \cdot \bar{\mu}_{\bar{\beta}}\right) \equiv \prod_{s=1}^{\bar{L}} \boldsymbol{z} \cdot h\left(\boldsymbol{\tau} \mid \bar{\mu}_{1, s} \bar{\mu}_{1, s^{\prime}} \ldots \bar{\mu}_{n, s} \bar{\mu}_{n, s^{\prime}}\right), \quad\left(\boldsymbol{z} \in \mathbb{Z}_{\alpha}\right)
$$

and take a sum over the entire orbit of $\mathbb{Z}_{\alpha}$.

Finally we define the function on the skeleton-faces by

$$
\mathbb{F}(\boldsymbol{\tau} \mid \nu)=N_{c}^{\bar{Z}(\boldsymbol{\tau})+C(\bar{\omega})} \sum_{\bar{\beta} \in \mathbf{C R}\left(\bar{\alpha}_{\bullet}\right)} \sum_{\boldsymbol{z} \in \mathbb{Z}_{\alpha}} \mathbf{F}\left(\boldsymbol{\tau} \mid \boldsymbol{z} \cdot \bar{\mu}_{\bar{\beta}}\right) .
$$

We count the powers of $N_{c}$ in the same way as (C.9) in section C. Namely, we compute $\theta=\theta(\boldsymbol{\tau})$ in (C.12) and count the number of cycles in $\bar{\omega}=\nu \theta$. The non-planarity inside open two-points also contribute through $\bar{Z}(\boldsymbol{\tau})$ in (C.28).

The $n$-point function of single-trace operators is written as

$$
G_{n}^{\prime}=\sum_{\ell \vdash L} \frac{1}{|\operatorname{Aut} V|} \sum_{\nu \in\left(S_{2 \bar{L}}^{\times \times}\right)_{\mathrm{phys}}} \sum_{\boldsymbol{\tau} \in S_{\boldsymbol{\tau}}} \mathbb{F}(\boldsymbol{\tau} \mid \nu) \delta\left(n_{\mathrm{ex}}, n\right) \delta\left(\left\{L_{\mathrm{ex}, i}\right\},\left\{L_{i}\right\}\right)
$$

where the sums over $\boldsymbol{\ell}$ and $\boldsymbol{\tau}$ are given in (C.20) and (C.22), respectively. We inserted two types of $\delta$-functions from (4.35). We sum the face permutation $\nu$ over the range (4.32). We used $G_{n}^{\prime}$ as in (C.27) because the last procedure removes two-point functions from $G_{n}$.

The fact that the powers of $N_{c}$ in (4.49) depend on the internal data $\boldsymbol{\tau}$ causes stratification, namely the skeleton graph of smaller genera contribute to the $1 / N_{c}$ corrections to the $n$-point functions at a fixed genus $[8,9,26]$.

\subsubsection{Sum over graphs}

We simplify (4.50) further in two steps. The first step is to take the connected part of $n$-point functions. This makes the subtle difference between $G_{n}$ and $G_{n}^{\prime}$ unimportant,

$$
\left(G_{n}^{\prime}\right)_{\text {connected }}=\left(G_{n}\right)_{\text {connected }}, \quad(\forall n \geq 3) .
$$

The second step is to interchange the order of summation in (4.50). Previously, we fixed the bridge length distribution $\ell \vdash L$ first, and summed over the skeleton graph with the 
edge lengths $\left\{\ell_{1}, \ell_{2}, \ldots, \ell_{\bar{L}}\right\}$. Now we fix the skeleton graph first, and distribute the bridge lengths later. Let us define a set of skeleton graphs with a given set of dimensions by

$$
\mathbf{S G}\left(L_{1}, L_{2}, \ldots, L_{n}\right)=\left\{\text { Skeleton graphs with } n \text { vertices } \mid \Delta(i \text {-th vertex })=L_{i}\right\} .
$$

The elements of $\mathbf{S G}\left(\left\{L_{i}\right\}\right)$ automatically solve the conditions (4.35). The mutually adjacent external Wick-contractions of (4.32) has disappeared by the definition of skeleton-ness.

Let $\Gamma \in \mathbf{S G}\left(\left\{L_{i}\right\}\right)$ and define

$$
\operatorname{Face}(\Gamma) \equiv \nu, \quad|\operatorname{Edge}(\Gamma)|=\bar{L}, \quad \operatorname{Vertex}(\Gamma)=\bar{\alpha}_{\bullet}, \quad|\operatorname{Vertex}(\Gamma)|=n .
$$

The $n$-point function (4.50) becomes

$$
\begin{gathered}
G_{n}^{\prime}=\sum_{\Gamma \in \mathbf{S G}\left(\left\{L_{i}\right\}\right)} \sum_{(\text {Distribute } \ell)} \frac{1}{|\operatorname{Aut} V(\boldsymbol{\ell})|} \sum_{\boldsymbol{\tau} \in S_{\boldsymbol{\tau}}} \mathscr{F}(\boldsymbol{\tau} \mid \Gamma) \\
\mathscr{F}(\boldsymbol{\tau} \mid \Gamma)=N_{c}^{\bar{Z}(\boldsymbol{\tau})+C(\bar{\omega})} \sum_{\boldsymbol{z} \in \mathbb{Z}_{\alpha}} \prod_{s=1}^{|\operatorname{Edge}(\Gamma)|} \boldsymbol{z} \cdot h\left(\boldsymbol{\tau} \mid \bar{\mu}_{1, s} \bar{\mu}_{1, s^{\prime}} \ldots \bar{\mu}_{n, s} \bar{\mu}_{n, s^{\prime}}\right)
\end{gathered}
$$

where $\bar{\omega}=\operatorname{Face}(\Gamma) \cdot \theta(\boldsymbol{\tau})$. The second sum of (4.54) is taken over the space of assigning a length to each edge of $\Gamma$,

$$
\begin{aligned}
& (\text { Distribute } \boldsymbol{\ell})=\left\{\operatorname{Map}: \operatorname{Edge}(\Gamma) \rightarrow \mathbb{Z}_{+}^{|\operatorname{Edge}(\Gamma)|} \mid\right. \\
& \left.\sum_{q=1}^{\bar{L}_{i}} \boldsymbol{\ell}_{i \mid q}=L_{i}, \bigsqcup_{i=1}^{n} \bigsqcup_{q=1}^{\bar{L}_{i}} \boldsymbol{\ell}_{i \mid q}=\left[1^{2 \boldsymbol{r}_{1}} 2^{2 \boldsymbol{r}_{2}} \ldots\right] \vdash 2 L, \quad \sum_{k} \boldsymbol{r}_{k}=|\operatorname{Edge}(\Gamma)|\right\} .
\end{aligned}
$$

The first sum of (4.54) is taken over partially labeled skeleton graphs. ${ }^{23}$ Note that we do distinguish the vertices having the same dimensions, and hence removed the sum over $\bar{\beta} \in \mathbf{C R}$ in (4.55). Recall that the sum over $\mathbf{C R}$ was needed in (4.37), because in the group-theory language a permutation $\bar{\alpha} \bullet$ cannot specify an ordered list uniquely. In the graph-theory language, we may distinguish the $i$-th and $j$-th vertices even if $L_{i}=L_{j}$.

Let us take the connected part of the $n$-point function and rewrite $\left(G_{n}\right)_{\text {connected }}$ further. We decompose the space $\mathbf{S G}\left(\left\{L_{i}\right\}\right)$ by the genus of a graph $\Gamma$,

$$
2-2 g=|\operatorname{Vertex}(\Gamma)|-|\operatorname{Edge}(\Gamma)|+|\operatorname{Faces}(\Gamma)| .
$$

We define the gauge theory moduli space $\mathcal{M}_{g, n}^{\text {gauge }}\left(\left\{L_{i}\right\}\right)$ by the first two sums of (4.54),

$$
\left(G_{n}\right)_{\text {connected }}=\sum_{g \geq 0} \sum_{\Gamma(\ell) \in \mathcal{M}_{g, n}^{\text {gauge }}\left(\left\{L_{i}\right\}\right)} \frac{1}{|\operatorname{Aut} V(\ell)|} \sum_{\boldsymbol{\tau} \in S_{\boldsymbol{\tau}}} \mathscr{F}(\boldsymbol{\tau} \mid \Gamma)
$$

where $\Gamma(\ell)$ is the skeleton graph whose bridge lengths are specified. Note that $g$ is a parameter of $\Gamma(\ell)$ which is not the powers of $N_{c}$ in $\left(G_{n}\right)_{\text {connected }}$ due to the stratification.

The skeleton graph with specific bridge lengths describing the connected $n$-point function can be identified as the so-called connected metric ribbon graphs. Using this connection, we will study the properties of $\mathcal{M}_{g, n}^{\text {gauge }}\left(\left\{L_{i}\right\}\right)$ in section 5 .

\footnotetext{
${ }^{23} \mathrm{By}$ a partially labeled skeleton graph, we mean that the $i$-th vertex of the graph has the dimensions $L_{i}$. We do not assign the flavor indices of the original operator $\mathcal{O}_{i}$ inside the $i$-th vertex.
} 


\section{Geometry from permutations}

We start our argument in an abstract way by looking at the equivalence between the space of connected metric ribbon graphs and the decorated moduli space. Then we define a subset of these spaces by imposing three conditions. We conjecture that this subspace is equal to the gauge theory moduli space $\mathcal{M}_{g, n}^{\text {gauge }}\left(\left\{L_{i}\right\}\right)$ introduced above, and discuss some examples.

\subsection{Ribbon graph and Riemann surface}

As explained in Introduction and appendix D.3, there is a one-to-one correspondence between the space of connected metric ribbon graphs (CMRG) with the decorated moduli space of Riemann surfaces,

$$
\mathrm{CMRG}_{g, n}=\mathcal{M}_{g, n} \times \mathbb{R}_{+}^{n} .
$$

In this equality, a graph defines a cell decomposition of a Riemann surface, $\Sigma_{g, n}=\sqcup_{i=1}^{n} R_{i}$. Each region $R_{i}$ contains a puncture of $\Sigma_{g, n}$, and two regions are separated by the edges of the graph. The edge lengths of the graph $\left\{\ell_{E}\right\}$ specify the complex structure of $\Sigma_{g, n}$.

We denote the sum of edge lengths around the $i$-th puncture by

$$
\sum_{E \in \partial R_{i}} \ell_{E}=p_{i} \in \mathbb{R}_{+}
$$

On $\Sigma_{g, n}$, l.h.s. is a cycle integral of a differential around the $i$-th puncture, and r.h.s. is the residue. In fact, for any $\left\{p_{1}, p_{2}, \ldots, p_{n}\right\} \in \mathbb{R}_{+}^{n}$, there exists a unique quadratic differential $\varphi$ having the residue $p_{i}$ at the $i$-th puncture, so-called the Jenkins-Strebel (JS) differential. The critical graph of $\varphi$ is isomorphic to the metric ribbon graph $\Gamma_{g, n}$. See reviews [47-49] for details.

Let us inspect the properties of ribbon graphs, constrained by the corresponding Riemann surfaces. If a ribbon graph completely triangulates a Riemann surface as $\Sigma_{g, n}=\sqcup_{i=1}^{n} R_{i}$, then the number of vertices, edges and faces are given by

$$
V_{g, n}^{\bullet}=2 n-4+4 g, \quad E_{g, n}^{\bullet}=3 n-6+6 g, \quad F_{g, n}^{\bullet}=n, \quad \text { (all trivalent). }
$$

Incomplete triangulations also constrains the graph data, as discussed in appendix D.2. If we focus on all possible ways of distributing lengths (a positive number) to each edge of the graph, we find

$$
\mathrm{CMRG}_{g, n} \simeq \mathbb{R}_{+}^{3 n-6+6 g}
$$

which is consistent with

$$
\operatorname{dim}_{\mathbb{R}} \mathcal{M}_{g, n}=2 n-6+6 g .
$$

This relation has been made precise in [17], where the integration measure of $\mathcal{M}_{g, n}$ is expressed by the edge length differentials $\left\{d \ell_{1}, d \ell_{2}, \ldots, d \ell_{3 n-6+6 g}\right\}$ under the constraints (5.2).

By definition, the space $\mathrm{CMRG}_{g, n}$ splits into the metric part and the ribbon graph part as

$$
\mathrm{CMRG}_{g, n}=\left(\text { Choice of }\left\{\ell_{E}>0\right\}\right) \times\left(\text { Choice of ribbon graph } \Gamma_{g, n}\right) .
$$


We can specify the ribbon graph uniquely by permutations. One way of doing this is

(Choice of ribbon graph $\Gamma_{g, n}$ )

$=($ Number of $k$-valent vertices $) \times($ Cyclic ordering of edges at each vertex $)$.

\subsection{Constraints from gauge theory}

We impose three constraints on $\mathcal{M}_{g, n}$ to select the gauge theory moduli space $\mathcal{M}_{g, n}^{\text {gauge }} \subsetneq$ $\mathcal{M}_{g, n}$.

The first condition is integrality, $\ell_{E} \in \mathbb{Z}_{+}$. As a corollary, the residue condition (5.2) becomes

$$
\sum_{E \in \partial R_{i}} \ell_{E}=L_{i} \in \mathbb{Z}_{+}
$$

which is equivalent to (4.15).

The second condition is the absence of self-contractions, $\ell_{i i}=0$. As a corollary, we obtain the extremality condition $\sum_{j \neq i} L_{j} \geq L_{i}$ for any $i$. This can be shown by

$$
\sum_{j \neq i} L_{j}-L_{i}=\sum_{j=1}^{n} L_{j}-2 L_{i}=\left(\ell_{i i}+2 \sum_{j \neq i} \ell_{j i}+\sum_{j \neq i} \sum_{k \neq i} \ell_{k j}\right)-2 \sum_{j} \ell_{j i}=-\ell_{i i}+\sum_{j \neq i} \sum_{k \neq i} \ell_{k j}
$$

which is non-negative if $\ell_{i i}=0$. Indeed, the correlator of scalar operators in gauge theory vanishes when the extremality condition is violated. The situation was different in matrix models, because self-contractions are allowed and $\ell_{i i}$ can be non-zero.

The above conditions also constrain the complex structure, which can be argued in two ways. One argument goes as follows. Since the complex structure is induced by decoration in the metric ribbon graph method, any inequality on the decoration parameters selects a proper subset of the moduli space. Thus we find

$$
\mathrm{CMRG}_{g, n}^{\triangle}=\left(\mathcal{M}_{g, n} \times \mathbb{Z}_{+}^{n}\right)^{\triangle}=\mathcal{M}_{g, n}^{\triangle} \times\left(\mathbb{Z}_{+}^{n}\right)^{\triangle}
$$

where $\left(\mathbb{Z}_{+}^{n}\right)^{\triangle}$ is the subspace of $\mathbb{Z}_{+}^{n}$ satisfying the generalized triangular inequality,

$$
\left(\mathbb{Z}_{+}^{n}\right)^{\triangle}=\left\{\left(L_{1}, L_{2}, \ldots, L_{n}\right) \in \mathbb{Z}_{+}^{n} \mid \sum_{j \neq i} L_{j} \geq L_{i} \quad(i=1,2, \ldots, n)\right\} .
$$

The moduli space without self-contractions $\mathcal{M}_{g, n}^{\Delta}$ is a subspace of $\mathcal{M}_{g, n}^{\triangle}$ according to (5.9). Another argument is based on the fact that Feynman graphs are dual to the critical graphs on Riemann surfaces, as discussed in appendix D.5. In general, the critical graph of Riemann surface may contain self-edges [17]. The self-edge is a boundary of the same face as shown in figure 11. The gauge theory moduli space $\mathcal{M}_{g, n}^{\text {gauge }}$ should not contain self-edges, because they correspond to the self-contractions in the dual Feynman graph [29].

The third condition is discrete homotopy, which is stronger than the usual homotopy in classifying edges. In classical geometry, we call two edges are homotopic if they end on the 

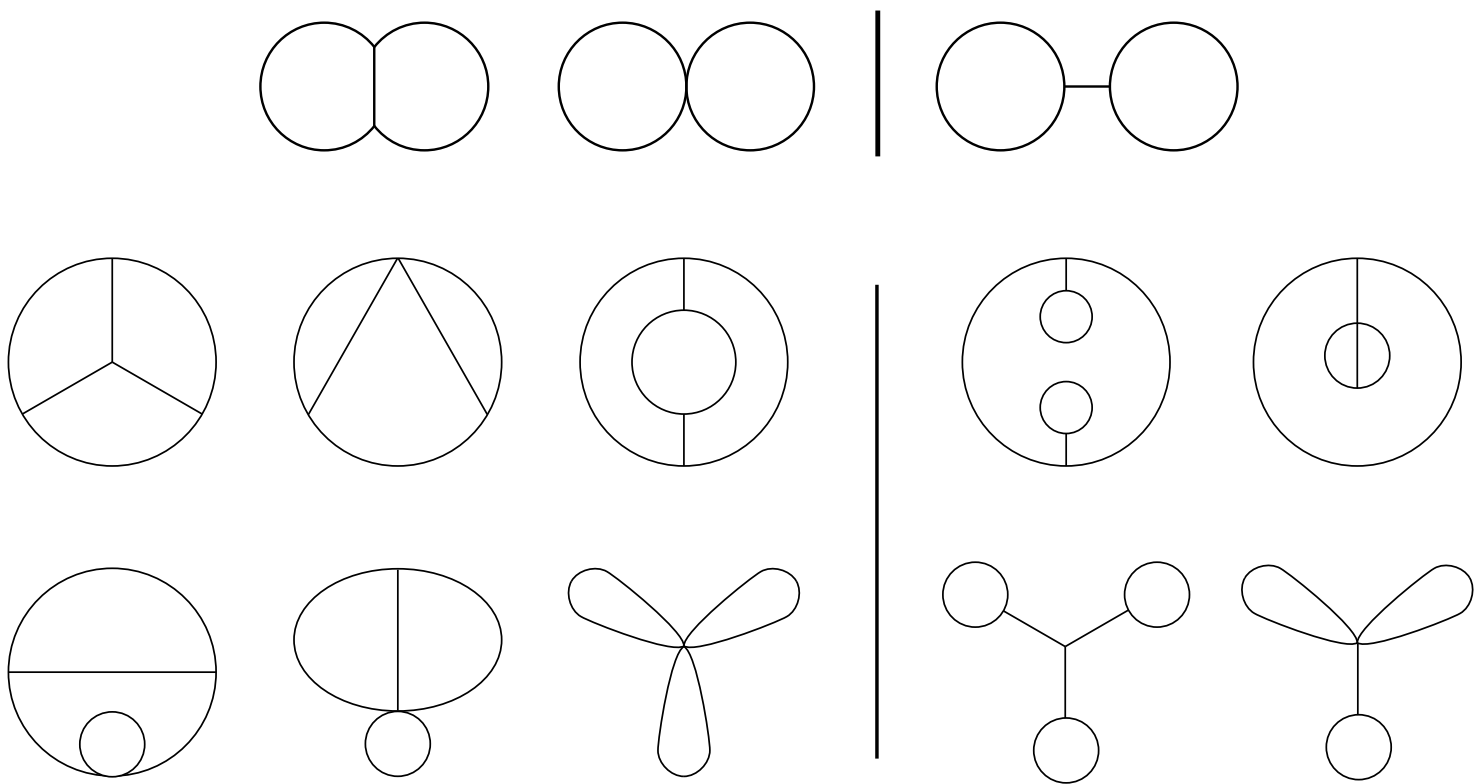

Figure 11. Examples of the connected metric ribbon graphs with $(g, n)=(0,3)$ (above) and $(0,4)$ (below). The diagrams on the right half have self-edges, and some diagrams on the left half are extremal.

same vertices and if they wind various one-cycles on $\Sigma_{g, n}$ in the same way. In our skeleton reduction, two edges are discrete-homotopic as long as they are consecutive and connect the same pair of operators, even if they cross each other. This condition corresponds to the exclusion of the mutually adjacent pairs discussed in appendix C.3.3. The equivalence under the discrete homotopy selects a subspace of the moduli space as

$$
\mathcal{M}_{g, n}^{\mathbf{\Lambda}, \text { disc }} \subset \mathcal{M}_{g, n}^{\mathbf{\Delta}} \subset \mathcal{M}_{g, n}
$$

Importantly, the discrete homotopy induces a special type of triangulation of Riemann surface. Since the discrete homotopy is stronger than the usual homotopy, our triangulation is simpler than those used in $[8,9] .{ }^{24}$

We conjecture that the restricted moduli space is equal to the gauge theory moduli space defined by the skeleton-reduced $n$-point formula $(4.58),{ }^{25}$

$$
\mathcal{M}_{g, n}^{\mathbf{\Lambda}, \text { disc }}=\mathcal{M}_{g, n}^{\text {gauge }}\left(\left\{L_{i}\right\}\right) .
$$

We see that l.h.s. indeed depends on $\left\{L_{i}\right\}$, because the minimal edge length is quantized. As a result, the length of external operators $\left\{L_{i}\right\}$ gives an upper bound on $g$. This property is quite different from the classical moduli space.

\footnotetext{
${ }^{24}$ This simplification may be useful if one is interested only in the contribution from the zero-length bridges in the hexagon methods.

${ }^{25}$ If we apply the skeleton reduction which assembles only the planar internal Wick-contractions, we should get $\mathcal{M}_{g, n}^{\triangle}=\mathcal{M}_{g, n}^{\text {gauge }}\left(\left\{L_{i}\right\}\right)$.
} 
Cell decomposition. Since $\mathcal{M}_{g, n}^{\text {gauge }}\left(\left\{L_{i}\right\}\right)$ is a set of metric ribbon graphs, it inherits the structure (5.6) and (5.7)

$$
\begin{aligned}
\mathcal{M}_{g, n}^{\text {gauge }}= & \left(\text { Choice of }\left\{\ell_{E} \geq 1\right\}\right) \times \\
& \times(\text { Number of } k \text {-valent vertices }) \times(\text { Cyclic ordering of edges at each vertex }) .
\end{aligned}
$$

When some of $\ell_{E}$ become zero, then vertices collide and the graph topology changes. In the literature, the classification of $\mathcal{M}_{g, n}^{\text {gauge }}$ by graph topology is called cell decomposition.

Consider the second factor of (5.14) in detail. Let us denote the valency set of the skeleton graph by $\mathrm{d}=\left[3^{d_{3}} 4^{d_{4}} \ldots\right]$, where $d_{k}$ is the number of $k$-valent vertices. From appendix D.2, we find that trivalent skeleton graphs have $d=\left[3^{2 n-4+4 g}\right]$, and the extremal skeleton graph with one vertex has $\mathrm{d}=\left[(2 n-2+4 g)^{1}\right]$. The valency set of general skeleton graphs at $(g, n)$ is in between,

$$
\mathrm{d} \in \mathrm{D}_{g, n}=\left\{\left[3^{2 n-4+4 g}\right],\left[3^{2 n-6+4 g} 4^{1}\right], \ldots,\left[3^{1}(2 n-3+4 g)^{1}\right],\left[(2 n-2+4 g)^{1}\right]\right\} .
$$

This pattern also constrains the number of edges in the skeleton graphs, denoted by $\bar{L}$ in section 4 . The trivalent graphs have $\bar{L}=3 n-6+6 g$ and the extremal graph has $n-1+2 g$. The number of edges in general skeleton graphs is

$$
\bar{L} \in \mathrm{L}_{g, n}=\{3 n-6+6 g, 3 n-7+6 g, \ldots, n+2 g, n-1+2 g\} .
$$

Generally there is no simple correspondence between the entries in (5.16) and (5.15). As shown in figure 11, the edges of a non-trivalent graph may end on the same vertex or different vertices, which changes the number of edges.

Classical limit of $\mathcal{M}_{\boldsymbol{g}, \boldsymbol{n}}^{\text {gauge }}$. The classical limit is defined by taking all bridge lengths to be large $\ell_{E} \gg 1$ with $g, n$ fixed, for the ribbon graphs whose faces are all triangles. We can neglect the quantization condition (5.8) in this limit. The second line of (5.14) is still a finite set, because the graph data are constrained by $(g, n)$ as in (5.15), (5.16). From (5.10) one expects that the dimensions of $\mathcal{M}_{g, n}^{\text {gauge }}\left(\left\{L_{i}\right\}\right)$ should agree with $\operatorname{dim}_{\mathbb{R}} \mathcal{M}_{g, n}$ as

$$
\mathcal{M}_{g, n}^{\text {gauge }}\left(\left\{L_{i}\right\}\right) \sim \mathbb{Z}^{2 n-6+6 g} .
$$

This equation can be understood in the graph theory language in appendix D.6. Owing to the relation (5.12), the space $\mathcal{M}_{g, n}^{\text {gauge }}$ remains a proper subset of $\mathcal{M}_{g, n}$ in the classical limit.

We expect that the generalized triangular inequality (5.11) disappears in more general setups. For example, when we include the covariant derivatives, then there are non-zero three-point functions with $\Delta_{1}>\Delta_{2}+\Delta_{3}$ due to the Laplacian $\square^{n}$. If we write $\Delta=\tau_{s}^{*}+s+$ $2 n$ and study the minimal twist $\tau^{\text {min }}$, then a similar triangular inequality was found in [50].

\subsection{Examples}

We compute the connected $n$-point functions $\left(G_{n}\right)_{\text {connected }}\left(\left\{L_{i}\right\}\right)$ for small $L=\frac{1}{2} \sum_{i} L_{i}$ and recast them into the form of (4.58). This procedure explicitly determines the space of reduced Wick-contractions $\mathcal{M}_{g, n}^{\text {gauge }}\left(\left\{L_{i}\right\}\right)$ for simple cases. 
We expand $\left(G_{n}\right)_{\text {connected }}$ in $1 / N_{c}$ and use $\xi=0,1,2, \ldots$ to count the powers of $N_{c}$, where $\xi=0$ corresponds to the planar term. For a given $\left\{L_{i}\right\}$, we define

$$
\begin{array}{r}
\mathrm{N}_{\xi, \lambda}^{\circ}\left(\left\{L_{i}\right\}\right)=\{\text { Feynman (or ribbon) graphs of genus } \xi \text { whose skeleton reduction } \\
\text { has the face permutation and of cycle type } \lambda\} .
\end{array}
$$

Different elements in $\mathrm{N}_{\xi, \lambda}^{\circ}$ have different cyclic orderings in (5.14), or different $\tau$. We introduce

$$
\mathrm{y}_{\xi, \lambda} \equiv \frac{\left|\mathrm{N}_{\xi, \lambda}^{\circ}\left(\left\{L_{i}\right\}\right)\right|}{\left|\mathbb{Z}_{\alpha}\right|}=\frac{\left|\mathrm{N}_{\xi, \lambda}^{\circ}\left(\left\{L_{i}\right\}\right)\right|}{\prod_{i=1}^{n} L_{i}} \in \mathbb{Q}
$$

which roughly counts the number of skeleton graphs whose face permutation of cycle type $\lambda$. This counting is not precise, because the action of $\mathbb{Z}_{\alpha}$ on Feynman graphs may have non-trivial fixed points, as discussed in appendix E. We write

$$
\begin{aligned}
\mathrm{N}_{\xi} & \equiv \sum_{\lambda} \mathrm{y}_{\xi, \lambda} \times \lambda \\
& =\mathrm{y}_{\xi, a}\left[3^{a_{3}} 4^{a_{4}} \ldots\right]+\mathrm{y}_{\xi, b}\left[3^{b_{3}} 4^{b_{4}} \ldots\right]+\mathrm{y}_{\xi, c}\left[3^{c_{3}} 4^{c_{4}} \ldots\right]+\ldots
\end{aligned}
$$

It will turn out that the lists $\mathrm{N}_{\xi}$ are perfectly consistent with our earlier classification (5.15). If $g$ represents the genus of the ribbon graph, $\nu_{\xi}$ may contain graphs of smaller genera, $\nu_{g}$ with $g<\xi$, owing to the stratification.

The details of the computation are explained in appendix E and in the attached supplementary material Mathematica notebook.

Three-point functions. We consider two examples, $\left(L_{1}, L_{2}, L_{3}\right)=(4,4,4)$ and $(6,3,3)$. For the first example, the list of the cycle type of face permutations is

$$
\mathrm{N}_{0}=\left[3^{2}\right], \quad \mathrm{N}_{1}=7\left[3^{2}\right]+6\left[3^{1} 7^{1}\right]+4\left[3^{2} 6^{1}\right], \quad \mathrm{N}_{2}=6\left[3^{1} 7^{1}\right]+3\left[12^{1}\right] .
$$

Figure 12 explains the origin of the first term of $\mathrm{N}_{0}, \mathrm{~N}_{1}$ in (5.21) in view of the original Feynman graphs. For the second example, the list is

$$
\mathrm{N}_{0}=\left[4^{1}\right], \quad \mathrm{N}_{1}=2\left[4^{1}\right]+6\left[8^{1}\right]+\frac{1}{3}\left[4^{3}\right], \quad \mathrm{N}_{2}=\left[4^{1}\right]+2\left[8^{1}\right]+\left[12^{1}\right] .
$$

The skeleton graphs appearing in $\left(G_{3}\right)_{\text {connected }}$ are shown in figures 13 and 14 .

These lists agree with the classification (5.15). For example, at genus one, we find

$$
\mathrm{D}_{1,3}=\left\{\left[3^{6}\right],\left[3^{4} 4^{1}\right],\left[3^{3} 5^{1}\right],\left[3^{2} 4^{2}\right],\left[4^{3}\right],\left[3^{1} 4^{1} 5^{1}\right],\left[3^{2} 6^{1}\right],\left[5^{2}\right],\left[4^{1} 6^{1}\right],\left[3^{1} 7^{1}\right],\left[8^{1}\right]\right\} .
$$

Some cycle types are missing in the actual examples, because there is no solution to the constraints on the quantized bridge lengths (5.8). We can represent each entry of $D_{1,3}$ as the toric diagram with three vertices as shown in figure 15 .

In general, the planar three-point functions have a unique skeleton graph. This is because the bridge lengths are completely fixed by the external operators

$$
\ell_{i j}=\frac{L_{i}+L_{j}-L_{k}}{2}, \quad\{i, j, k\} \in\{1,2,3\}
$$



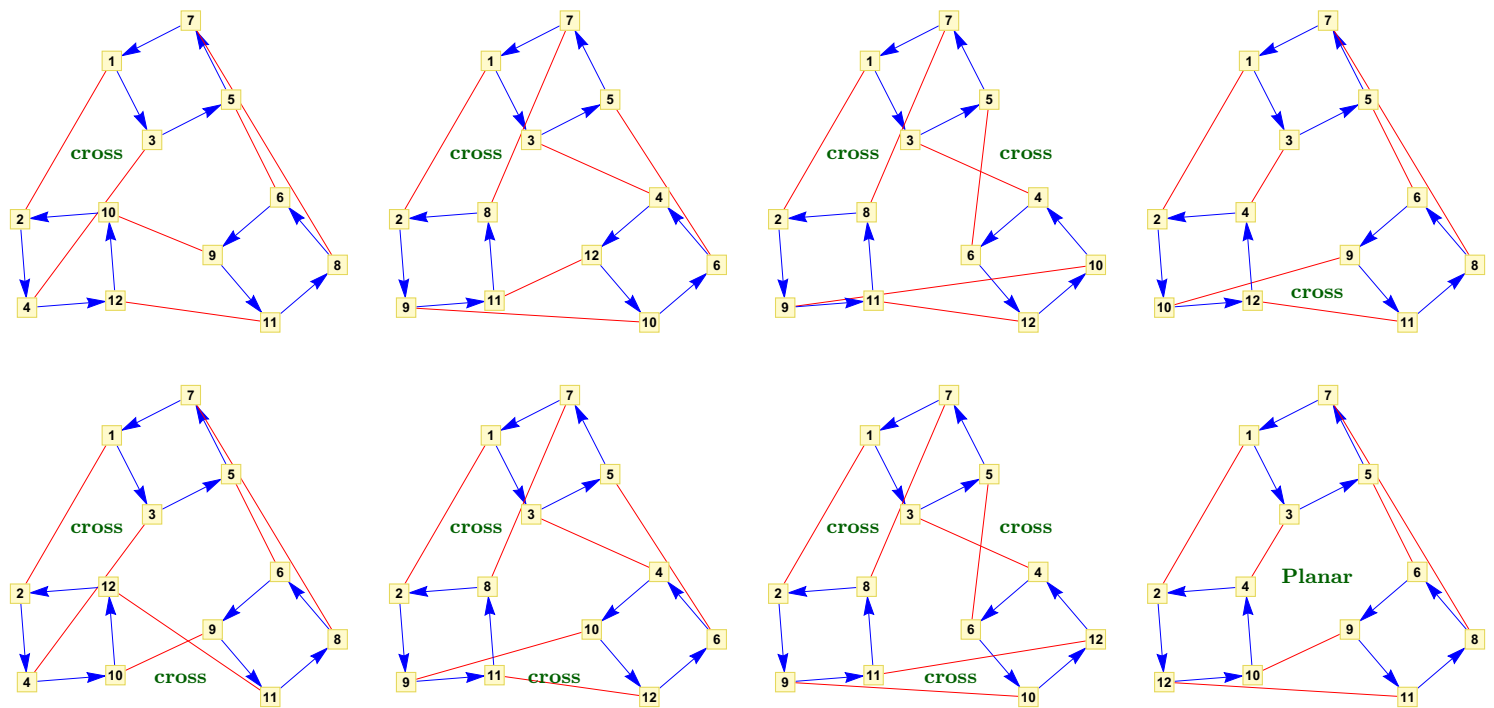

Figure 12. Original Feynman graphs corresponding to $7 \times\left[3^{2}\right] \in \mathrm{N}_{1}$ and $\left[3^{2}\right] \in \mathrm{N}_{0}$ in $(5.21)$. The first 7 graphs are part of the leading non-planar corrections to the three-point function, while the last graph is planar. All of them gives $\nu \in\left[3^{2}\right]$ after the skeleton reduction of section 4 .
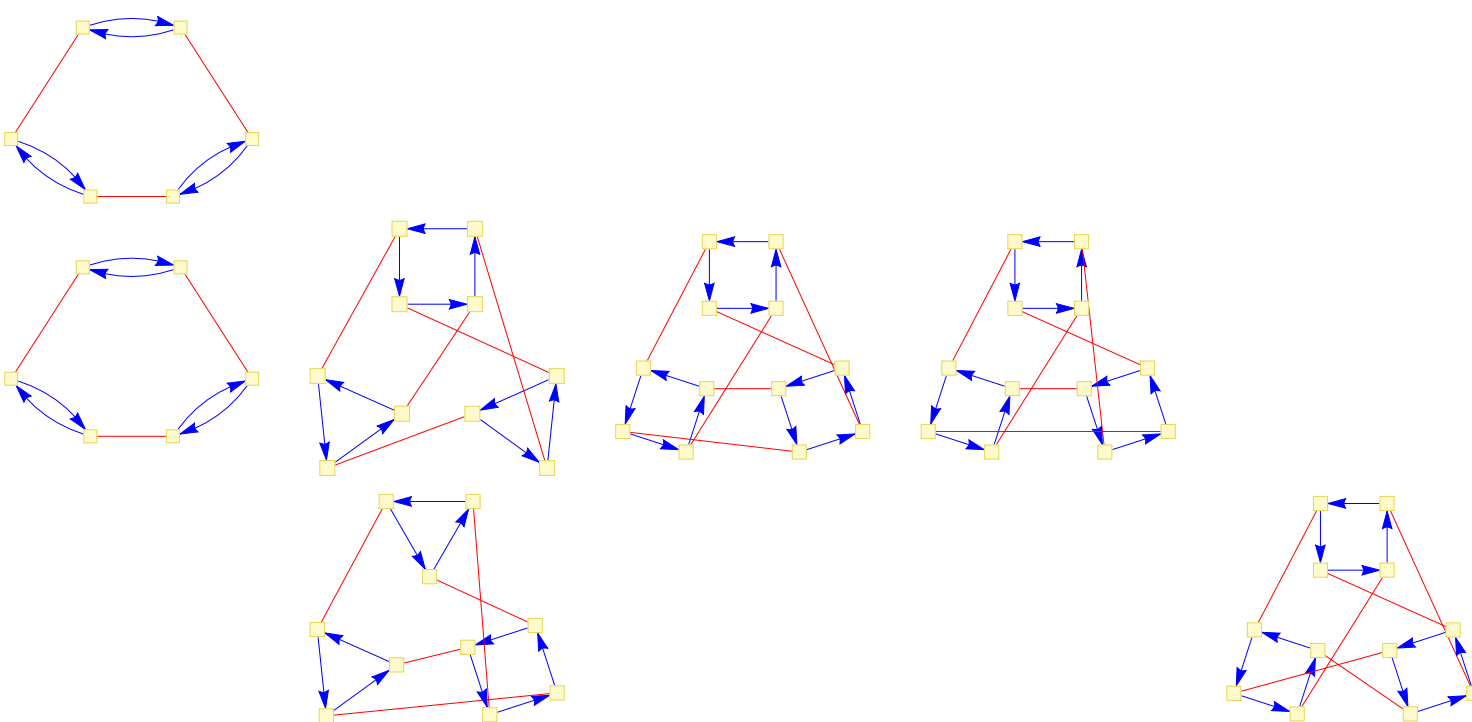

Figure 13. Skeleton graphs of $\left(G_{3}\right)_{\text {connected }}(4,4,4)$. The top, middle and bottom row corresponds to $\xi=0,1,2$, respectively. 

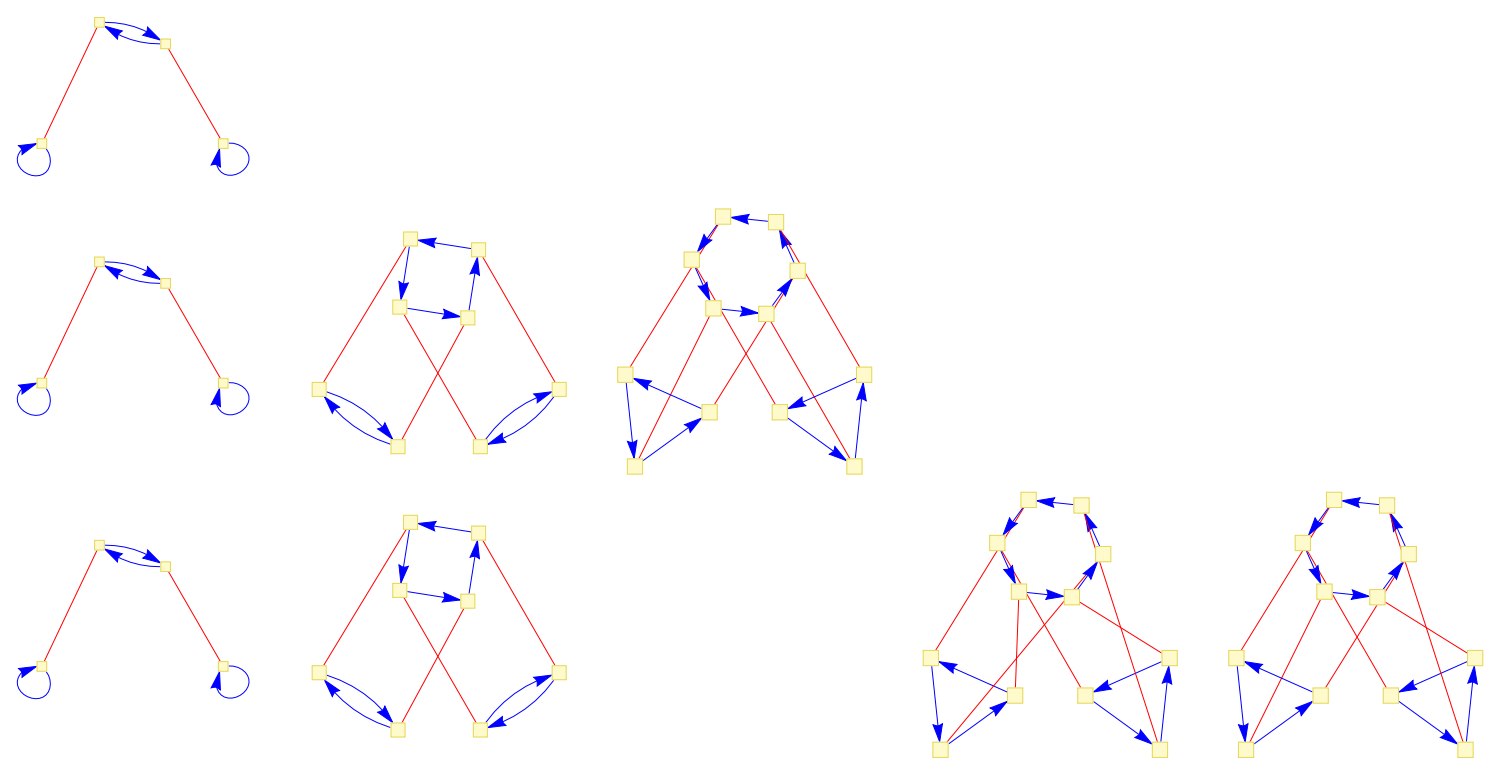

Figure 14. Skeleton graphs of $\left(G_{3}\right)_{\text {connected }}(6,3,3)$.
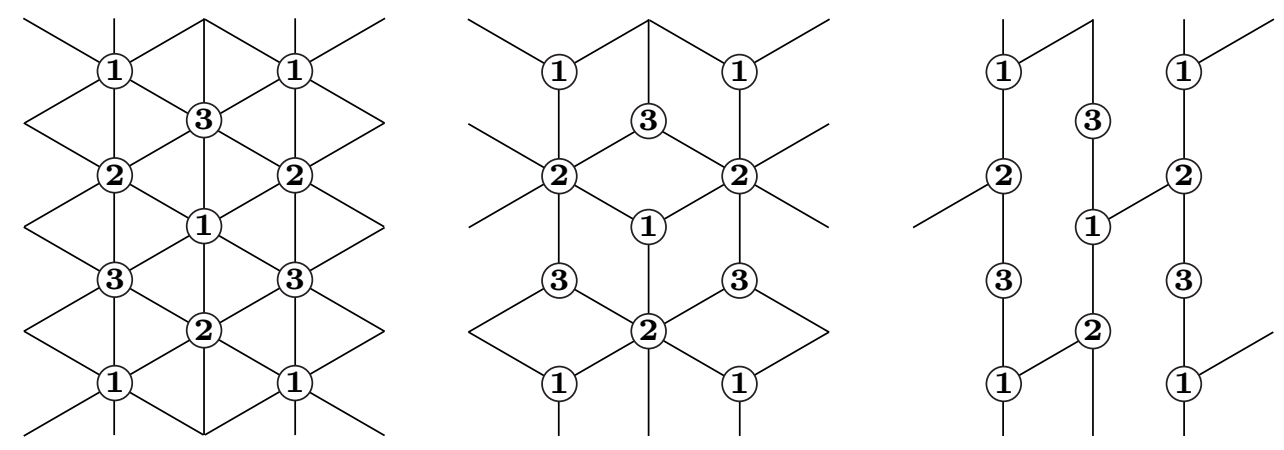

Figure 15. Examples of toric diagrams in $\mathcal{M}_{1,3}^{\text {gauge }}$, representing $[\nu]=\left[3^{6}\right],\left[4^{3}\right]$, $\left[8^{1}\right]$. The diagram in the center is extremal and has $\ell_{13}=0$.

and the cyclic orderings are fixed uniquely. Thus we have

$$
\mathcal{M}_{0,3}^{\text {gauge }}\left(L_{1}, L_{2}, L_{3}\right)=\{p t\}
$$

up to the change of graph topology in the extremal limit.

Another interesting observation is that the skeleton graph of $\left(G_{3}\right)_{\text {connected }}(L, L, 2)$ is unique irrespective of the genus. By taking first the Wick-contraction of $\mathcal{O}_{3}$ with $\mathcal{O}_{1}, \mathcal{O}_{2}$, we find that the remaining Wick-contractions are same as the two-point function of length $L-1$, whose graph topology is trivial.

Four-point functions. We consider three examples, $\left(L_{1}, L_{2}, L_{3}, L_{4}\right)=$ $(3,3,3,3),(6,2,2,2)$ and $(6,4,2,2)$. For the first example, the list of the cycle type of face permutations is

$$
\mathrm{N}_{0}=6\left[4^{2}\right]+2\left[3^{4}\right], \quad \mathrm{N}_{1}=18\left[4^{2}\right]+6\left[4^{1} 8^{1}\right]+8\left[3^{1} 9^{1}\right]
$$


and for the second example, the list is

$$
\mathrm{N}_{0}=2\left[6^{1}\right]+3\left[4^{2}\right], \quad \mathrm{N}_{1}=6\left[10^{1}\right]+3\left[4^{1} 8^{1}\right]+\left[6^{2}\right] .
$$

The last example is more complicated,

$$
\begin{aligned}
\mathrm{N}_{0}= & 6\left[3^{1} 5^{1}\right]+4\left[3^{2} 4^{1}\right] \\
\mathrm{N}_{1}= & 22\left[10^{1}\right]+14\left[5^{1} 7^{1}\right]+30\left[3^{1} 5^{1}\right]+16\left[3^{1} 9^{1}\right] \\
& +4\left[3^{1} 5^{1} 6^{1}\right]+4\left[4^{1} 8^{1}\right]+2\left[3^{2} 8^{1}\right]+4\left[3^{1} 4^{1} 7^{1}\right]+4\left[3^{2} 4^{1}\right] \\
N_{2}= & 12\left[10^{1}\right]+20\left[14^{1}\right]+4\left[5^{1} 7^{1}\right]+4\left[3^{1} 9^{1}\right] .
\end{aligned}
$$

The skeleton graphs appearing in $\left(G_{4}\right)_{\text {connected }}$ are shown in figures 16-18. Again, these lists are consistent with (5.15). For example, at $g=1$ we get

$$
\mathrm{D}_{1,4}=\left\{\left[3^{8}\right], \ldots,\left[4^{4}\right], \ldots,\left[6^{2}\right], \ldots,\left[10^{1}\right]\right\}
$$

The planar four-point functions have several skeleton graphs. The moduli space can be written as

$$
\mathcal{M}_{0,4}^{\text {gauge }}\left(\left\{L_{i}\right\}\right)=\left\{\left(\xi_{1}, \xi_{2}\right) \in \mathbb{Z}^{2} \mid \ell_{i j} \geq 0\right\} \times\{\text { Two choices in cyclic ordering }\} .
$$

The first factor comes from the sum over bridge lengths given by

$$
\left(\begin{array}{rrr}
\ell_{12} & \ell_{13} & \ell_{14} \\
& \ell_{23} & \ell_{24} \\
& \ell_{34}
\end{array}\right) \mapsto\left(\begin{array}{ccc}
\ell_{12}+\xi_{1} & \ell_{13}+\xi_{2} & \ell_{14}-\xi_{1}-\xi_{2} \\
& \ell_{23}-\xi_{1}-\xi_{2} & \ell_{24}+\xi_{2} \\
& & \ell_{34}+\xi_{1}
\end{array}\right), \quad\left(\xi_{1}, \xi_{2}\right) \in \mathbb{Z}^{2}
$$

The second factor comes from the ordering of $\left(\mathcal{O}_{1}, \mathcal{O}_{2}, \mathcal{O}_{3}, \mathcal{O}_{4}\right)$. Suppose we take the open two-point between $\mathcal{O}_{1}, \mathcal{O}_{2}$ as

$$
\left(p \sim p+\ell_{12}-1\right) \quad \text { from } \mathcal{O}_{1}, \quad\left(q \sim q+\ell_{12}-1\right) \text { from } \mathcal{O}_{2}
$$

where $p, q$ can be any number owing to the cyclic symmetry. Then, the subsequent color index $p+\ell_{12}$ of $\mathcal{O}_{1}$ is contracted either by $\mathcal{O}_{3}$ or $\mathcal{O}_{4}$,

$$
\left\{\begin{array}{lll}
\left(p+\ell_{12} \sim p+\ell_{12}+\ell_{13}-1\right) & \text { from } \mathcal{O}_{1} \text { for contracting } & \left(\mathcal{O}_{1}, \mathcal{O}_{3}\right) \\
\left(p+\ell_{12} \sim p+\ell_{12}+\ell_{14}-1\right) & \text { from } \mathcal{O}_{1} \text { for contracting } & \left(\mathcal{O}_{1}, \mathcal{O}_{4}\right) .
\end{array}\right.
$$

This choice determines the skeleton graph completely, from the planarity and cyclic symmetry. For example, the first line of (5.33) means that the color index $q+\ell_{12}$ of $\mathcal{O}_{2}$ must be contracted with $\mathcal{O}_{4}$. Graphically, $\left(\mathcal{O}_{1}, \mathcal{O}_{2}, \mathcal{O}_{3}, \mathcal{O}_{4}\right)$ are placed at the left, right, top, bottom white circles in figure 26 in the counterclockwise ordering. 

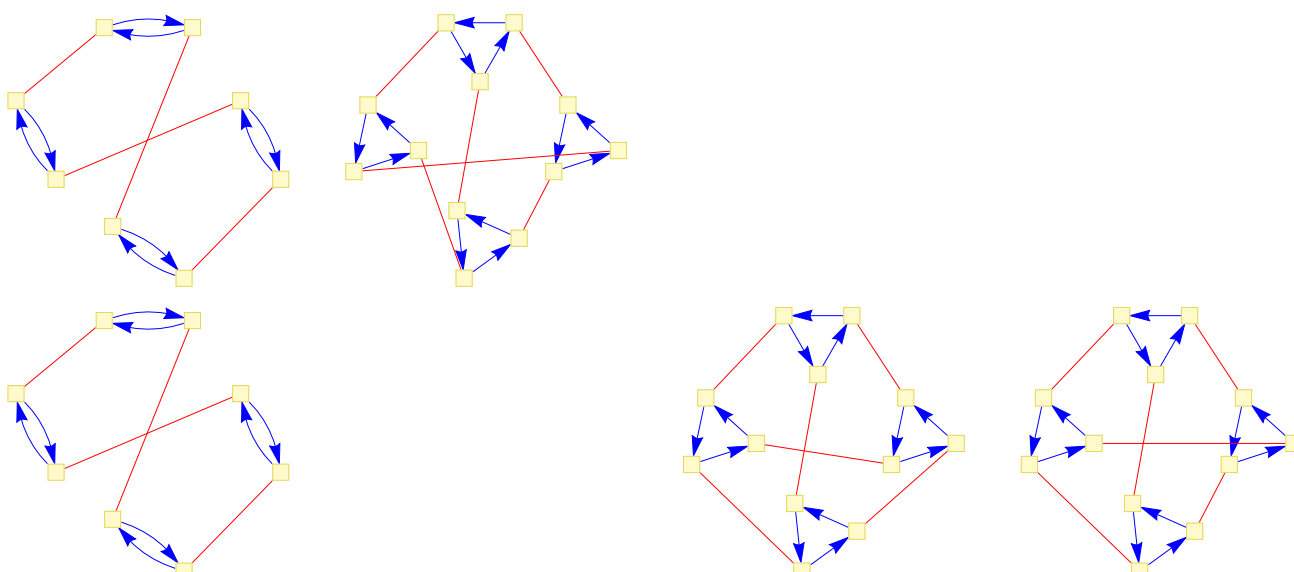

Figure 16. Skeleton-reduced diagrams of $\left(G_{4}\right)_{\text {connected }}(3,3,3,3)$.
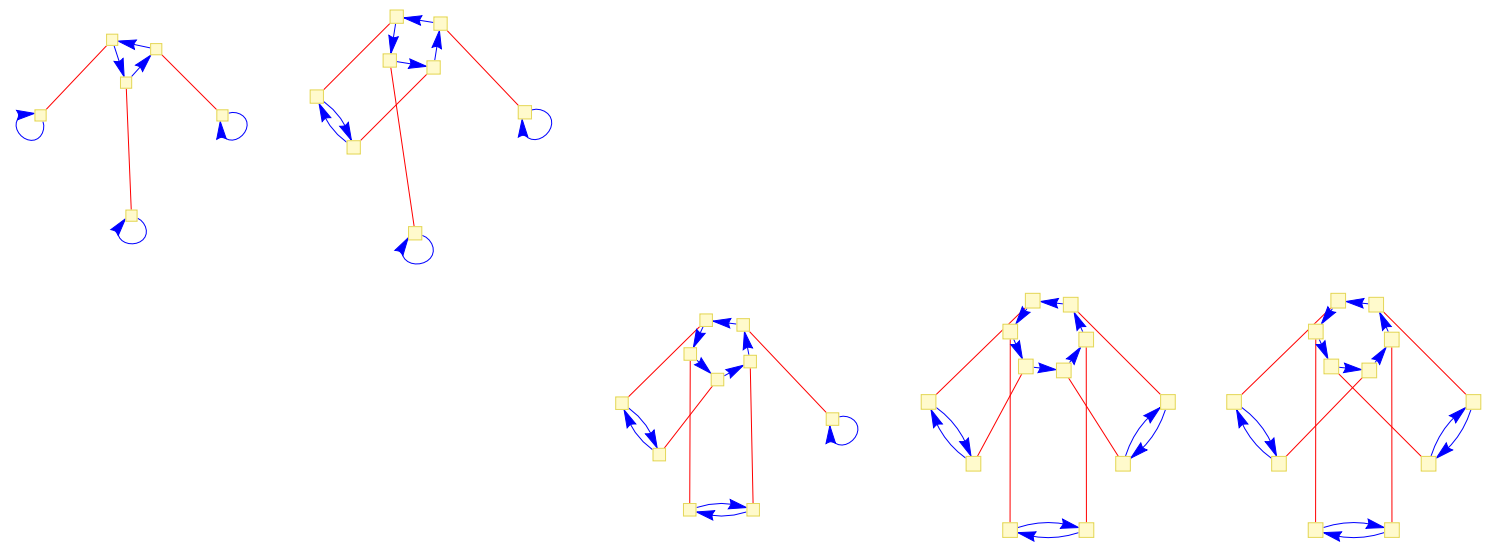

Figure 17. Skeleton-reduced diagrams of $\left(G_{4}\right)_{\text {connected }}(6,2,2,2)$.

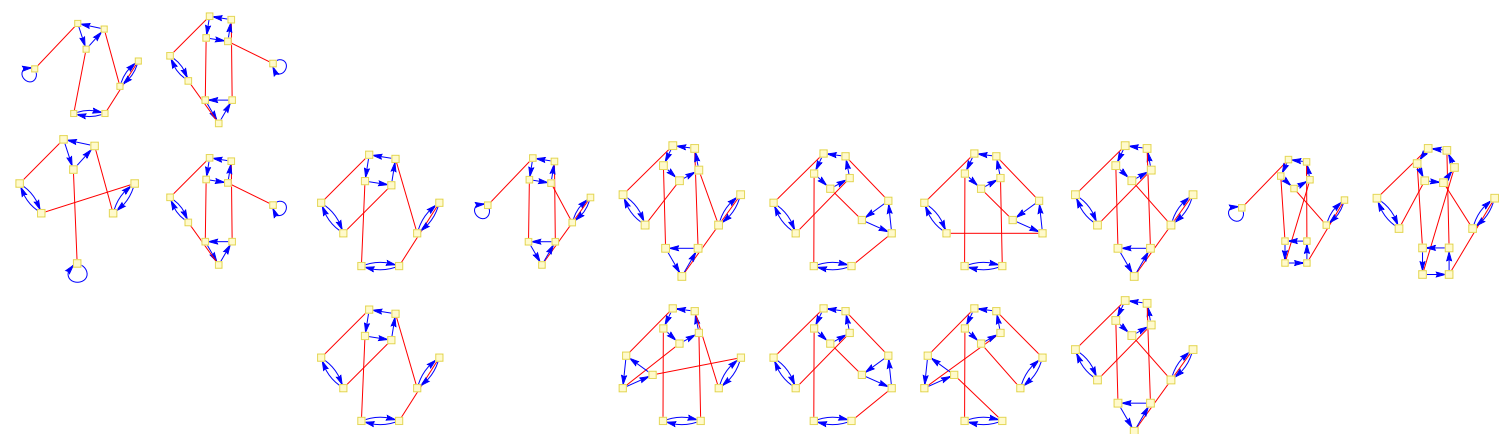

Figure 18. Part of the skeleton-reduced diagrams of $\left(G_{4}\right)_{\text {connected }}(6,4,2,2)$. There are 28 diagrams in total. 


\section{Metric ribbon graphs in string theory}

We briefly discuss how metric ribbon graphs show up in string theory.

In the light-cone SFT, closed string worldsheet is characterized by the GiddingsWolpert quadratic differential [51], whose analytic structure is classified by Nakamura graphs [52]. This situation is in parallel with the JS differential and its critical graph. The moduli space of light-cone worldsheet theory can be regarded as the space of Wickcontractions in the Hermitian matrix model [53], described by permutations [54]. The light-cone and conformal methods differ in many ways. For example, Nakamura graphs consist of quadrivalent vertices (four-point interactions) only. Furthermore, the GiddingsWolpert differential introduces a global time coordinate on $\Sigma_{g, n}$, whereas the JS differential does not have such a quantity.

In conformal gauge, we argue that the metric ribbon graphs arise from the analytic properties of the classical energy-momentum tensor, and characterize the permutation of Stokes sectors of string worldsheet on $\mathrm{AdS}_{5} \times \mathrm{S}^{5}$.

As discussed in appendix D, the JS quadratic differential dual to a Feynman diagram of $n$-point functions has the analytic structure

$$
\varphi=-\frac{L_{i}^{2}}{4 \pi^{2}} \frac{d z^{2}}{\left(z-z_{i}\right)^{2}}+O\left(\left|z-z_{i}\right|^{-1}\right), \quad(i=1,2, \ldots, n) .
$$

It also has $2 n-4+4 g$ simple zeroes somewhere on $\Sigma_{g, n}$. This behavior is similar to that of the classical worldsheet energy-momentum tensor

$$
T(z) \sim \frac{\kappa_{i}}{\left(z-z_{i}\right)^{2}}+O\left(\left|z-z_{i}\right|^{-1}\right), \quad(i=1,2, \ldots, n) .
$$

Here $\left\{\kappa_{i}\right\}$ is related to the conformal dimensions of vertex operators. If we interpret $T(z)$ as the "JS" differential, then the parameters $\left\{\kappa_{i}\right\}$ determine the complex structure of the underlying Riemann surface. Physically, we may replace the Strebel condition by other conditions like the equations of motion, as long as the external parameters uniquely fix the quadratic differential and its critical graph. ${ }^{26}$

In AdS/CFT, the three-point functions of heavy operators in $\mathcal{N}=4 \mathrm{SYM}$ at strong coupling have been studied by Pohlmeyer reduction [56-59], borrowing the techniques developed for the gluon scattering [60-63]. The Pohlmeyer reduction on $S^{3}$ gives us the Hitchin system,

$$
\left[\partial+B_{z}, \bar{\partial}+B_{\bar{z}}\right]=0 .
$$

The $\mathrm{SL}(2, \mathbb{C})$ flat connections $\left(B_{z}, B_{\bar{z}}\right)$ can be written as [58]

$$
\begin{aligned}
& B_{z}=\frac{\Phi_{z}}{\zeta}+A_{z}=\frac{1}{\zeta}\left(\begin{array}{cc}
0 & -\frac{\sqrt{T}}{2} e^{-i \gamma} \\
-\frac{\sqrt{T}}{2} e^{i \gamma} & 0
\end{array}\right)+\left(\begin{array}{cc}
-\frac{i \partial \gamma}{2} & \frac{\rho e^{i \gamma}}{\sqrt{T} \sin 2 \gamma} \\
\frac{\rho e^{-i \gamma}}{\sqrt{T} \sin 2 \gamma} & \frac{i \partial \gamma}{2}
\end{array}\right) \\
& B_{\bar{z}}=\zeta \Phi_{\bar{z}}+A_{\bar{z}}=\zeta\left(\begin{array}{cc}
0 & \frac{\sqrt{\bar{T}}}{2} e^{i \gamma} \\
\frac{\sqrt{\bar{T}}}{2} e^{-i \gamma} & 0
\end{array}\right)+\left(\begin{array}{cc}
\frac{i \bar{\partial} \gamma}{2} & \frac{\tilde{\rho} e^{-i \gamma}}{\sqrt{\bar{T}} \sin 2 \gamma} \\
\frac{\tilde{\rho} e^{i \gamma}}{\sqrt{\bar{T}} \sin 2 \gamma} & -\frac{i \bar{\partial} \gamma}{2}
\end{array}\right)
\end{aligned}
$$

\footnotetext{
${ }^{26}$ Non-Strebel critical graphs are considered in [55].
} 
where $T(z), \bar{T}(\bar{z})$ are the worldsheet energy-momentum tensors, $\zeta=\frac{1-x}{1+x}$ is the spectral parameter, and $(\gamma, \rho, \tilde{\rho})$ are the $\mathrm{SO}(4)$-invariant degrees of freedom after the Pohlmeyer reduction. The flatness condition (6.3) is equivalent to the compatibility of the auxiliary linear problem

$$
\left(\partial+B_{z}\right) \psi=\left(\bar{\partial}+B_{\bar{z}}\right) \psi=0 .
$$

These have two linearly independent solutions, called small and large depending on the asymptotics of $|z| \rightarrow \infty$ with $\arg (z)$ fixed. When we rotate $z$ the asymptotic behavior of the solutions changes. The region of the common asymptotics is called the Stokes sector.

In the semi-classical analysis, we uniformize the worldsheet by $d w=\sqrt{T(z)} d z$, which introduces branch points at the simple zeroes of $T(z) .{ }^{27}$ If we regard $T(z)$ as a quadratic differential on $\Sigma_{g, n}$, then the number of simple zeroes in $T(z)$ is given by (D.6). We can draw a metric ribbon graph by connecting the zeroes and the poles of $T(z)$. This graph knows how Stokes sectors are permuted around the branch points. A similar argument has been done in the light-cone string theory in $[53,54]$.

\section{Conclusion and outlook}

We studied the general $n$-point functions of scalar multi-trace operators in $\mathcal{N}=4 \mathrm{SYM}$ with $\mathrm{U}\left(N_{c}\right)$ gauge group at tree-level. We obtained permutation-based formulae valid for any $n$ and to all orders of $1 / N_{c}$. The edge-based formula is interpreted as the topological partition function on $\Sigma_{0, n}$ with defects, which naturally decomposes into pairs of pants. We applied a new skeleton reduction to find another set of formulae. The skeleton-reduced Feynman graphs generate metric ribbon graphs, which form a subset of the moduli space of complex structure of Riemann surfaces. Our skeleton reduction stratifies the moduli space with respect to the genus, whose top component is simpler than the usual skeleton reduction.

We find open/closed duality from the $n$-point functions of gauge-invariant operators. The pants decomposition resembles closed-string interaction, while the triangulation of $\Sigma_{g, n}$ through the skeleton reduction resembles open-string interaction.

This work hopefully initiates several future directions of research, which we want to revisit in the near future.

The first direction is to generalize our formulae into the full sector of $\mathcal{N}=4 \mathrm{SYM}$, and to include the $g_{\mathrm{YM}}$ loop corrections. Perturbatively, the loop corrections are computed by taking the OPE with the insertion of interaction terms in the Lagrangian, and integrating over the insertion point in spacetime. The integrand of $n$-point functions at $\ell$-loop can be rewritten roughly as the $(n+\ell)$-point function at tree-level. Pants decomposition in section 3 may be useful to deal with such quantities.

The second direction is to compare our results with the BPS correlators derived by the $S^{4}$ localization [64-66]. Whether one can recast the generating function of BPS correlators as the $\tau$-function of an integrable system is an interesting question.

\footnotetext{
${ }^{27}$ In the scattering problem of $n$ gluons on $\mathrm{AdS}_{3}$, one chooses $d w=\sqrt{p_{n}(z)} d z$ where $p_{n}(z)$ is a polynomial of degree $n-2$. The number of coefficients of $p_{n}(z)$ is equal to the number of cross-ratios, or $\operatorname{dim}_{\mathbb{C}} \mathcal{M}_{0, n}=$ $n-3$. After integration we find $w \sim z^{n / 2}$, showing that there are $n$ Stokes sectors at each half-plane.
} 
The third direction is to apply our formalism to the determinant-like operators, or equivalently the LLM geometry [67]. It has recently been conjectured that certain states on the LLM backgrounds are isomorphic to those of $\mathcal{N}=4 \mathrm{SYM}$, and the correlators are related by the redefinition of $N_{c}$ [68]. This conjecture may be checked by rewriting our results in an appropriate representation basis.

The last direction is to employ our Wick-contractions techniques for studying integrable systems in the free-field representation $[69,70]$. The skeleton reduction in section 4 is analogous to the hexagon program [7-9]. Then, the pants decomposition in section 3 may give a hint on the three-point functions or related form-factors in terms of Quantum Spectral Curve along the line of [71, 72].

\section{Acknowledgments}

RS thanks Hiroyuki Fuji and Sanjaye Ramgoolam for discussions and comments on the manuscript.

\section{A Notation}

We denote an indexed set $\alpha_{1}, \alpha_{2}, \ldots$ collectively by $\left\{\alpha_{i}\right\}$. A multiple sum or product is written as

$$
\sum_{\alpha_{1}} \sum_{\alpha_{2}} \ldots f\left(\alpha_{1}, \alpha_{2}, \ldots\right)=\sum_{\left\{\alpha_{i}\right\}} f\left(\left\{\alpha_{i}\right\}\right)
$$

The tensor product of groups is written as $\otimes_{i} G_{i}$, or as $\prod_{i} G_{i}$ when any elements of $G_{i}$ and $G_{j}(i \neq j)$ commute.

\section{A.1 Permutations}

A cyclic permutation is denoted by $\left(i_{1} i_{2} \ldots i_{\ell}\right)$, and $(i)(j) \ldots$ represents an identity element. ${ }^{28}$ We define the permutation action by

$$
\sigma:\{v(1), \ldots, v(n)\} \mapsto\left\{v^{\prime}(1), \ldots, v^{\prime}(n)\right\}, \quad v^{\prime}(\sigma(n))=v(n) .
$$

For example, we have

$$
\sigma=(123): v=\{a, b, c\} \mapsto v^{\prime}=\{c, a, b\}
$$

as represented in figure 19. We find the identity

$$
\alpha \cdot \beta(n)=\alpha(\beta(n)), \quad \alpha\left(i_{1} i_{2} \ldots i_{\ell}\right) \alpha^{-1}=\left(\alpha\left(i_{1}\right) \alpha\left(i_{2}\right) \ldots \alpha\left(i_{\ell}\right)\right) .
$$

In Mathematica, the group multiplication $\alpha \cdot \beta$ is implemented by PermutationProduct $[\beta, \alpha]$.

The $\delta$-function on the symmetric group is defined by

$$
\delta_{L}(\sigma)= \begin{cases}1 & \left(\sigma=1 \in S_{L}\right) \\ 0 & \text { (otherwise) }\end{cases}
$$

\footnotetext{
${ }^{28}$ Usually we do not put commas inside the bracket for permutation cycles.
} 

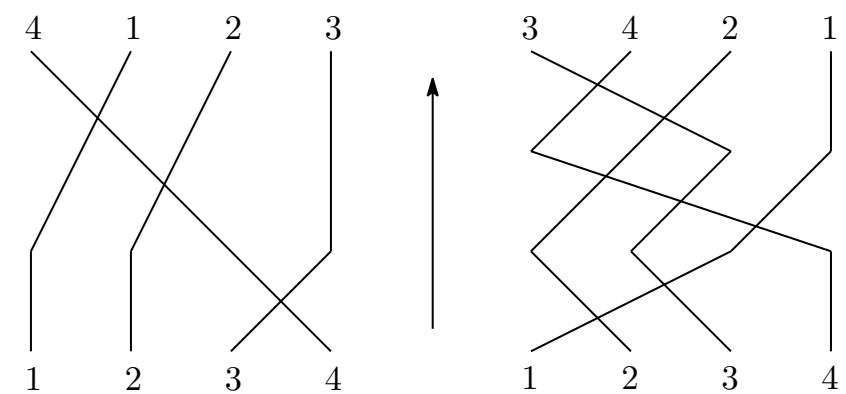

Figure 19. Multiplication of permutations represented as diagrams, showing (123)(34) $=(1234)$ and $(123)(1234)(132)=(2314)$.

We denote the conjugacy class of $\alpha$ by $[\alpha]$,

$$
[\alpha] \equiv \frac{1}{L !} \sum_{\sigma \in S_{L}} \sigma^{-1} \alpha \sigma .
$$

Generally, two conjugacy classes commute; $\left[\alpha_{1}\right]\left[\alpha_{2}\right]=\left[\alpha_{2}\right]\left[\alpha_{1}\right]$. The conjugacy class of $S_{L}$ is characterized by its cycle type, or equivalently a partition of $L$. We denote the partition of a positive integer by

$$
\lambda \equiv\left[1^{\lambda_{1}} 2^{\lambda_{2}} \ldots L^{\lambda_{L}}\right] \vdash L
$$

which means

$$
\sum_{k=1}^{L} k \lambda_{k}=L, \quad \sum_{k=1}^{L} \lambda_{k} \equiv C(\lambda) .
$$

Here $C(\lambda)$ counts the number of cycles in $\lambda \in S_{L}$, e.g.

$$
C(\text { identity })=C((1)(2)(3) \ldots(L))=L, \quad C((12))=C((12)(3) \ldots(L))=L-2 .
$$

Often we use $[\alpha]$ to signify the cycle type of $\alpha$.

The wreath product group is denoted by $S_{L}[G]$, which is a composition of $G^{\otimes L}$ and $S_{L}$ permuting the order of the copies of $G$ 's. The order of this group is given by

$$
\left|S_{L}[G]\right|=L !|G|^{L} .
$$

For example, the action of $S_{L}\left[\mathbb{Z}_{2}\right]$ on $L$ pairs is generated by $\left(\left\{\pi_{i}\right\}, \sigma\right)$, where

$$
\mathbb{Z}_{2}^{\otimes L} \ni \pi_{i}:\left(a_{2 i-1}, a_{2 i}\right) \mapsto\left(a_{2 i}, a_{2 i-1}\right), \quad S_{L} \ni \sigma:\left(a_{2 k-1}, a_{2 k}\right) \mapsto\left(a_{2 \sigma(k)-1}, a_{2 \sigma(k)}\right) .
$$

The stabilizer subgroup is denoted by

$$
\operatorname{Stab}_{G}(x)=\{g \in G \mid g(x)=x\} \subset G .
$$

Here $g(x)$ can be any group action, such as $g(x)=g x g^{-1}$. By the orbit-stabilizer theorem, the number of inequivalent elements of the group coset is given by $|G| /\left|\operatorname{Stab}_{G}(x)\right|$. 


\section{A.2 Permutation basis of operators}

We express a multi-trace operator of length $L$ as the equivalence class in the permutation group $S_{L}$. Let $\mathcal{O}_{\alpha}^{\vec{A}}$ be a scalar multi-trace operator,

$$
\mathcal{O}_{\alpha}^{A_{1} A_{2} \ldots A_{L}}=\operatorname{tr}_{L}\left(\alpha \Phi^{A_{1}} \Phi^{A_{2}} \ldots \Phi^{A_{L}}\right) \equiv \sum_{a_{1}, a_{2} \ldots, a_{L}=1}^{N_{c}}\left(\Phi^{A_{1}}\right)_{a_{\alpha(1)}}^{a_{1}}\left(\Phi^{A_{2}}\right)_{a_{\alpha(2)}}^{a_{2}} \ldots\left(\Phi^{A_{L}}\right)_{a_{\alpha(L)}}^{a_{L}}
$$

Here $\alpha \in S_{L}$ defines the color structure of $\mathcal{O}_{\alpha}^{\vec{A}}$. If $\alpha \in \mathbb{Z}_{L}$, then $\mathcal{O}_{\alpha}^{\vec{A}}$ becomes a single-trace operator, usually denoted by

$$
\mathcal{O}_{\alpha}^{\vec{A}}=\operatorname{tr}\left(\Phi^{A_{1}} \Phi^{A_{2}} \ldots \Phi^{A_{L}}\right), \quad\left(\alpha \in \mathbb{Z}_{L}\right) .
$$

For later purposes, we introduce another notation for the same operator by

$$
\mathcal{O}_{\alpha}^{A_{1} A_{2} \ldots A_{L}}=\left(\prod_{p=1}^{L} \delta_{a_{\alpha(p)}}^{a_{p^{\prime}}}\right) \sum_{\left\{a_{p}, a_{p^{\prime}}\right\}}\left(\Phi^{A_{1}}\right)_{a_{1^{\prime}}}^{a_{1}}\left(\Phi^{A_{2}}\right)_{a_{2^{\prime}}}^{a_{2}} \ldots\left(\Phi^{A_{L}}\right)_{a_{L^{\prime}}}^{a_{L}} .
$$

The $\delta$-function prefactor can be represented by another permutation

$$
\iota_{\alpha} \equiv \prod_{p=1}^{L}\left(\alpha(p) p^{\prime}\right) \in \mathbb{Z}_{2}^{\otimes L}
$$

We can reorder the flavor indices in r.h.s. of (A.13), which leads to the gauge symmetry,

$$
\mathcal{O}_{\alpha}^{A_{1} A_{2} \ldots A_{L}}=\mathcal{O}_{\gamma^{-1} \alpha \gamma}^{A_{\gamma(1)} A_{\gamma(2)} \ldots A_{\gamma(L)}}, \quad\left(\forall \gamma \in S_{L}\right) .
$$

If we neglect the spacetime dependence, the scalar fields obey the $\mathrm{U}\left(N_{c}\right)$ Wick-contraction rule,

$$
\left(\Phi^{A}\right)_{a}^{b}\left(x_{i}\right)\left(\Phi^{B}\right)_{c}^{d}\left(x_{j}\right)=g^{A B}\left(x_{i j}\right) \delta_{a}^{d} \delta_{c}^{b}, \quad g^{A B}\left(x_{i j}\right)=\tilde{g}^{A B}\left|x_{i}-x_{j}\right|^{-2}
$$

where $\tilde{g}^{A B}=\delta^{A B}$ if $\Phi^{A}$ is a real scalar of $\mathcal{N}=4 \mathrm{SYM}$. If the gauge group is $\mathrm{SU}\left(N_{c}\right)$, we replace the scalar fields of $\mathrm{SU}\left(N_{c}\right)$ theory by those of $\mathrm{U}\left(N_{c}\right)$ theory as

$$
\left(\Phi^{\mathrm{SU}\left(N_{c}\right)}\right)_{a}^{b} \equiv\left(\Phi^{\mathrm{U}\left(N_{c}\right)}\right)_{a}^{b}-\frac{\delta_{a}^{b}}{N_{c}} \operatorname{tr}\left(\Phi^{\mathrm{U}\left(N_{c}\right)}\right) .
$$

Let us write the two-point functions of scalar operators of $\mathcal{N}=4 \mathrm{SYM}$ as

$$
G_{2}\left(\alpha_{1}, \alpha_{2}\right)=\left\langle\mathcal{O}_{\alpha_{1}}\left(x_{1}\right) \mathcal{O}_{\alpha_{2}}\left(x_{2}\right)\right\rangle, \quad\left(\alpha_{1}, \alpha_{2} \in S_{L}\right) .
$$

The r.h.s. is given by the sum over all possible Wick-contractions,

$$
\begin{aligned}
G_{\alpha_{1} \alpha_{2}} & =\left\langle\prod_{p=1}^{L}\left(\Phi^{A_{p}}\right)_{a_{\alpha_{1}(p)}}^{a_{p}}\left(x_{1}\right) \prod_{q=1}^{L}\left(\Phi^{B_{q}}\right)_{b_{\alpha_{2}(q)}}^{b_{q}}\left(x_{2}\right)\right\rangle \\
& =\left.\sum_{\sigma \in S_{L}} \prod_{p, q=1}^{L} g^{A_{p} B_{q}}\left(x_{12}\right) \delta_{b_{\alpha_{2}(q)}}^{a_{p}} \delta_{a_{\alpha_{1}(p)}}^{b_{q}}\right|_{q=\sigma^{-1}(p)} .
\end{aligned}
$$


The product over the color indices can be simplified by relabeling,

$$
\prod_{p=1}^{L} \delta_{b_{\alpha_{2} \sigma^{-1}(p)}}^{a_{p}} \delta_{a_{\alpha_{1}(p)}^{\sigma^{-1}(p)}}^{b_{1}}=\prod_{p=1}^{L} \delta_{b_{\alpha_{2} \sigma^{-1}(p)}}^{a_{p}} \prod_{r=1}^{L} \delta_{a_{\alpha_{1}(r)}}^{b_{\sigma^{-1}(r)}}=\prod_{p=1}^{L} \delta_{a_{\alpha_{1} \sigma \alpha_{2} \sigma^{-1}(r)}}^{a_{p}}
$$

where we used $\alpha_{2} \sigma^{-1}(p)=\sigma^{-1}(r)$. This gives

$$
G_{\alpha_{1} \alpha_{2}}=\sum_{\sigma \in S_{L}}\left(\prod_{p} g^{A_{\sigma^{-1}(p)} B_{p}}\right) \delta_{L}\left(\Omega \alpha_{1} \sigma^{-1} \alpha_{2} \sigma\right)
$$

where the $\delta$-function is given in (A.5). The symbol $\Omega$ is an element of the group algebra $\mathbb{C}\left[S_{L}\right]$

$$
\Omega \equiv \sum_{\omega \in S_{L}} N_{c}^{C(\omega)} \omega^{-1}
$$

where $C(\omega)$ is in (A.8). Thanks to the property $C(\omega)=C\left(\gamma \omega \gamma^{-1}\right)$, the $\Omega$ is a class function

$$
\Omega=\gamma \Omega \gamma^{-1} \quad\left(\forall \gamma \in S_{L}\right)
$$

\section{A.3 Feynman diagrams in the double-line notation}

The space of Wick-contractions $\mathcal{W}$ represents the sum over all Feynman diagrams. In the double-line notation, Feynman diagrams become ribbon graphs owing to the cyclic ordering at each vertex. The graph data of vertices, edges, and faces uniquely specifies a ribbon graph. Thus we introduce a triple of permutations to represent the graph data: ${ }^{29}$

$$
\text { (Vertices, Edges, Faces) } \leftrightarrow \quad(\alpha, W, \omega) \in\left(S_{2 L}, \mathbb{Z}_{2}^{\otimes L}, S_{2 L}\right), \quad \omega=\alpha W .
$$

Here the vertex permutation $\alpha$ knows the color structure of external operators $\left\{\mathcal{O}_{i}\right\}$, and the face permutation $\omega$ knows the color-index loops. By using permutations, we can represent the Feynman graph as the Cayley graph of the composition of $(\alpha, W) .{ }^{30}$

The Feynman graphs in the double-line notation can be understood as a Cayley graph in the following way. We rewrite the permutation basis of operators in the double-index notation as in (A.15). Now a scalar field carries one flavor half-edge and two color halfedges. All half-edges are paired by the pair of involutions $\left(\iota_{\alpha}, W\right)$ with $\iota_{\alpha}=\prod_{i=1}^{n} \iota_{\alpha_{i}}$ in (A.16). The Cayley graph of the composition of $\left(\iota_{\alpha}, W\right)$ is the usual Feynman graph in the double-line notation; see figure 20.

The Feynman graphs in the double-line notation have the ribbon graph structure. A ribbon graph is a graph with cyclic ordering at each vertex, which is represented by $\alpha$ or $\omega$ in our setup.

\footnotetext{
${ }^{29}$ The permutations $(\alpha, W, \omega)$ are denoted by $\left(\sigma_{0}, \sigma_{1}, \sigma_{\infty}\right)$ in [36].

${ }^{30}$ The Cayley graph $\Gamma(G)$ is a colored directed graph, defined canonically from the generators of a finite group $G$.
} 


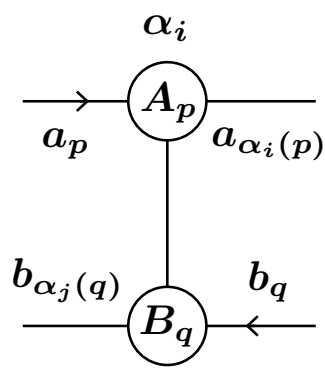

$\alpha_{j}$

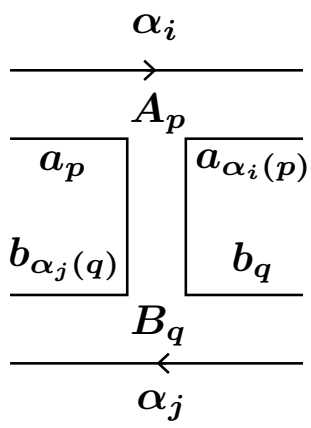

Figure 20. (Left) Usual Feynman graph as the Cayley graph of $(\alpha, W)$. (Right) The Feynman graph in the double-line notation as the Cayley graph of $\left(\iota_{\alpha}, W\right)$, representing the Wick-contraction $\left\langle\left(\Phi^{A_{p}}\right)_{a_{\alpha_{i}(p)}}^{a_{p}}\left(\Phi^{B_{q}}\right)_{b_{\alpha_{j}(q)}}^{b_{q}}\right\rangle$.

\section{A.4 Summary of skeleton reduction}

Let us present an overview of our notation in section 4, where we introduced various different symbols for making our argument straightforward.

The number of all Wick-contractions between $\mathcal{O}_{i}$ and $\mathcal{O}_{j}$ is denoted by $\left\{\ell_{i j}\right\}$ in (1.3). We introduce two more symbols as the refinement of $\left\{\ell_{i j}\right\}$ in (4.15) and (C.13). The first one gives a partition of $\ell_{i j}$ where each segment represents the consecutive sets of Wick-edges between $\mathcal{O}_{i}$ and $\mathcal{O}_{j}$

$$
\ell_{i i}^{(\rho)}=0, \quad \ell_{i j}^{(\rho)}=\ell_{j i}^{(\rho)}, \quad \ell_{i j}=\sum_{\rho=1}^{r_{i j}} \ell_{i j}^{(\rho)}, \quad \sum_{i<j}^{n} \sum_{\rho=1}^{r_{i j}} \ell_{i j}^{(\rho)}=L .
$$

The second one defines a partition of $L_{i}$ and of the operator $\mathcal{O}_{i}$,

$$
\ell_{i} \equiv\left(\ell_{i \mid 1}, \ell_{i \mid 2}, \ldots, \ell_{i \mid \bar{L}_{i}}\right) \vdash L_{i}, \quad L_{i}=\sum_{q=1}^{\bar{L}_{i}} \ell_{i \mid q} .
$$

Again each segment represents the consecutive Wick-contractions. Hence, both symbols are relabeling of the same object. The collection of $\left\{\boldsymbol{\ell}_{i}\right\}$ gives a partition of $2 L$

$$
\bigsqcup_{i=1}^{n} \ell_{i}=\left[1^{2 r_{1}} 2^{2 r_{2}} \ldots\right] \vdash 2 L
$$

We take half of this partition and define

$$
[\ell]=\left[1^{\boldsymbol{r}_{1}} 2^{\boldsymbol{r}_{2}} \ldots\right] \vdash L, \quad \sum_{k} \boldsymbol{r}_{k}=\bar{L}
$$

where $\boldsymbol{r}_{k}$ is the number of consecutive Wick-contractions with $k$ fields. The partition $[\ell] \vdash L$ is related to the skeleton reduction, and should not be confused by the cycle type of $\alpha=\prod_{i} \alpha_{i}$ denoted by $\lambda=\left[1^{\lambda_{1}} 2^{\lambda_{2}} \ldots\right] \vdash 2 L$. An example of $\ell_{i j}^{(\rho)}$ is illustrated in figure 21 , and another example of $\ell_{i}$ is given in figure 23 . 
One definition of the skeleton reduction is to assemble scalar fields in $\mathcal{O}_{i}$ into small groups. Let us regroup the color (or flavor) indices as

$$
p \in\left\{1,2, \ldots, L_{i}\right\}=\left\{(\mathrm{p}, \bar{p}) \mid \bar{p} \in\left\{1,2, \ldots, \ell_{\mathrm{p}}\right\}, \mathrm{p} \in\left\{1,2, \ldots, \bar{L}_{i}\right\}\right\}
$$

where $\mathrm{p}$ labels the open end-points and $\bar{p}$ labels the internal indices as

$$
\mathcal{O}_{i}=\prod_{p=1}^{L_{i}}\left(\Phi^{A_{p}}\right)_{a_{\alpha_{i}(p)}}^{a_{p}} \stackrel{\text { partition }}{\longrightarrow} \quad \overline{\mathcal{O}}_{i}=\prod_{\mathrm{p}=1}^{\bar{L}_{i}}\left(\boldsymbol{\Phi}_{p}^{(i)}\right)_{a_{\bar{\alpha}_{i}(\mathrm{p})}}^{a_{\mathrm{p}}}=\prod_{\mathrm{p}=1}^{\bar{L}_{i}} \prod_{\bar{p}=1}^{\ell_{\mathrm{p}}}\left(\Phi^{A_{\mathrm{p}, \bar{p}}}\right)_{a_{\mathrm{p}+\bar{p}}}^{a_{\mathrm{p}}+\bar{p}}
$$

We call $\overline{\mathcal{O}}_{i}$ a reduced operator, and $\boldsymbol{\Phi}_{\mathrm{p}}^{(i)}$ a sequence or sequential fields. Each $\boldsymbol{\Phi}_{\mathrm{p}}^{(i)}$ consists of $\ell_{\mathrm{p}}$ consecutive fields. There are many ways to create a reduced operator from $\mathcal{O}_{i}$. We call $\left\{\overline{\mathcal{O}}_{i}\right\}$ a partition of $\mathcal{O}_{i}$.

We impose the following rule for a pair of sequential fields,

$$
\begin{aligned}
& \left(\boldsymbol{\Phi}^{\boldsymbol{A}_{p+1 \sim p+\ell}^{(i)}}\right)_{a_{p+\ell+1}}^{a_{p+1}}\left(\boldsymbol{\Phi}^{\boldsymbol{A}_{q+1 \sim q+\ell^{\prime}}^{(j)}}\right)_{b_{q+\ell^{\prime}+1}}^{b_{q+1}} \\
& \quad=\left.\delta_{\ell, \ell^{\prime}} \sum_{\bar{W}} \prod_{k=1}^{\ell} g^{A_{p+k} B_{q+k^{\prime}}} \delta_{b_{q+k^{\prime}+1}}^{a_{p+k}} \delta_{a_{p+k+1}}^{b_{q+k^{\prime}}}\right|_{q+k^{\prime}=\bar{W}^{-1}(p+k)}
\end{aligned}
$$

which we call an open two-point function. Using the $\delta$-functions of color indices in the open two-point function, we define two functions $R$ and $Z$ as

$$
\prod_{k=1}^{\ell} \delta_{b_{j} \bar{W}^{-1}(p+k)}^{a_{p+k}} \delta_{a_{\alpha_{i}(p+k)}}^{b_{\bar{W}-1}(p+k)} \equiv N_{c}^{Z\left(\alpha_{i} \bar{W} \alpha_{j} \bar{W}^{-1} \mid a_{p+1}, b_{q+1}\right)} R_{a_{p+\ell+1} b_{q+\ell+1}}^{a_{p+1} b_{q+1}}
$$

The properties of these functions are studied at the end of section 4.1.

After the skeleton reduction, the Wick-contractions are taken in two steps. The first step is to take all pairs of sequential fields $\left(\boldsymbol{\Phi}_{\mathrm{p}}^{(i)}, \boldsymbol{\Phi}_{\mathrm{q}}^{(j)}\right)$, and the second step is to take the sum inside open two-points. Correspondingly, the pairing map $W \in \mathbb{Z}_{2}^{\otimes L}$ in (2.7) can be rewritten as

$$
W \ni\left(a_{p}, b_{q}\right)=\left(a_{\mathbf{p}, \bar{p}}, b_{\mathbf{q}, \bar{q}}\right) \quad \leftrightarrow \quad\left(a_{\mathbf{p}}, b_{\mathbf{q}}\right) \times(\bar{p}, \bar{q}) \in(V, \tau) .
$$

We call $V$ an external Wick-contraction, and $\tau$ an internal Wick-contraction.

We write the union of the double-index $(\mathrm{p}, \bar{p})$ as

$$
P \in\{1,2, \ldots, 2 L\}=\left\{(\mathrm{P}, \bar{p}) \mid \bar{p} \in\left\{1,2, \ldots, \ell_{\mathrm{P}}\right\}, \mathrm{P} \in\{1,2, \ldots, 2 \bar{L}\}\right\} .
$$

The symbol $\mathrm{P}$ describes the partitions of $\left\{\mathcal{O}_{i}\right\}$,

$$
\{1,2, \ldots, 2 \bar{L}\} \ni \mathrm{P} \quad \leftrightarrow \quad\left(\mathrm{p}_{1}, \mathrm{p}_{2}, \ldots, \mathrm{p}_{n}\right), \quad \mathrm{p}_{i} \in\left\{1,2, \ldots, \bar{L}_{i}\right\}, \quad \sum_{i=1}^{n} \bar{L}_{i}=2 \bar{L} .
$$


The skeleton-edges can be represented in two notations, $\mathrm{P}=1,2, \ldots, 2 \bar{L}$ and $E=$ $1,2, \ldots, \bar{L}$. The symbol $E$ is rewriting of $(i j, \rho)$,

$$
\sum_{E=1}^{\bar{L}} \ell_{E}=L, \quad \sum_{j \neq i} r_{i j}=\bar{L}_{i}, \quad \sum_{i<j} r_{i j}=\bar{L} .
$$

The symbol $\mathrm{P}$ is a double copy of $E$. For example, we find the identity

$$
E \sim(\mathrm{P}, \mathrm{Q}), \quad \tau_{\mathrm{P}}=\tau_{\mathrm{Q}} \quad \Leftrightarrow \quad\left(a_{\mathrm{P}} a_{\mathrm{Q}}\right) \in V
$$

\section{B Another face-based formula}

In section 2.3 we derived a face-based formula of $n$-point functions. Below we discuss another formula by using the rims labeled by $\alpha$, as illustrated in figure 3. It turns out that the constraint $\delta_{2 L}\left(\alpha^{-1} \omega W\right)$ disappears in this labeling, but another constraint must be imposed.

Let us denote the cycle type of $\omega$ by $[\omega]=\left[1^{w_{1}} 2^{w_{2}} \ldots\right] \vdash 2 L$,

$$
C(\omega)=\sum_{f} w_{f}, \quad 2 L=\sum_{f} f w_{f} .
$$

The Feynman graph has $w_{f}$ faces with $f$ edges, and $C(\omega)$ color-index loops. We have $w_{1}=0$ because the self-contractions are forbidden. Each face defines a cyclic translation by going around the boundary of the face counterclockwise. We can parametrize $\omega$ as a product of cyclic permutations,

$$
\omega=\prod_{R=1}^{C(\omega)} \Phi_{R}=\prod_{R=1}^{C(\omega)}\left(\phi_{R, 1} \phi_{R, 2} \ldots \phi_{R, f_{R}}\right)
$$

or using $\left\{w_{f}\right\}$ as

$$
\omega=\left(\phi_{1,1}^{(2)} \phi_{1,2}^{(2)}\right)\left(\phi_{2,1}^{(2)} \phi_{2,2}^{(2)}\right) \ldots\left(\phi_{w_{2}, 1}^{(2)} \phi_{w_{2}, 2}^{(2)}\right)\left(\phi_{1,1}^{(3)} \phi_{1,2}^{(3)} \phi_{1,3}^{(3)}\right) \ldots\left(\phi_{w_{3}, 1}^{(3)} \phi_{w_{3}, 2}^{(3)} \phi_{w_{3}, 3}^{(3)}\right) \ldots
$$

The face permutation $\omega$ written as sequences of rims define Wick-contractions. For example,

$$
\omega \ni(\ldots, p, q, r \ldots) \Rightarrow W \ni\left(p, \alpha^{-1}(q)\right)\left(q, \alpha^{-1}(r)\right) .
$$

For each factor inside $\omega$ in (B.2), we introduce the function on faces by

$$
\mathbb{F} \cdot \Phi_{R}=N_{c}\left(\prod_{k=1}^{f_{R}} h^{A_{\phi_{R, k}}, A_{\alpha-1}\left(\phi_{R, k+1}\right)}\right)^{1 / 2}, \quad\left(\alpha=\prod_{i=1}^{n} \alpha_{i} \in S_{2 L}\right)
$$

and write $n$-point functions as $G_{n}=\sum_{\omega} \mathbb{F}(\omega)$.

Let us sum over $\omega$ in two steps. The first sum is over the cycle type $w \vdash 2 L$ and the second sum over $\omega$ 's at a fixed cycle type. The latter sum can be generated by

$$
\mathcal{W}(w) \equiv\left\{\gamma \omega_{0} \gamma^{-1} \mid \gamma \in S_{2 L} / \operatorname{Aut}\left(\omega_{0}\right)\right\}, \quad \operatorname{Aut}\left(\omega_{0}\right)=\prod_{f \geq 1} S_{w_{f}}\left[\mathbb{Z}_{f}\right]
$$


where $\omega_{0}$ is a fixed (or representative) permutation with the cycle type $w$. The division by Aut $\left(\omega_{0}\right)$ removes the redundancy of relabeling in (B.3).

The space $\mathcal{W}(w)$ in (B.6) contains various types of unphysical Wick-contractions. The first type is self-contractions. This happens when two consecutive numbers $(\ldots, p, q \ldots) \in$ $\omega$ come from the same operator, $(p, q) \in \alpha_{i}$, or when $\omega$ contains one-cycles, $(p) \ni \omega$. We remove the self-contractions by the property of $h^{A B}$ in (2.16). The second type comes from the faces with the wrong orientation. According to our definition of $\mathbb{F}$ in (B.5), two cycles which differ by orientation, $\left(\phi_{1} \phi_{2} \ldots \phi_{f}\right)$ and $\left(\phi_{f} \phi_{f-1} \ldots \phi_{1}\right)$, produce different flavor factors. We should choose the orientation of each face so that they are consistent with the global choice. When wrongly oriented faces are glued together, the square-root on $h^{A B}$ in (B.5) remains unresolved. In terms of permutations, this condition for the consistent orientation can be rephrased as

$$
\omega \ni(\ldots p, \alpha(q) \ldots) \Leftrightarrow \omega \ni(\ldots q, \alpha(p) \ldots), \quad(\forall p, q)
$$

where the two sequences $\ldots p, \alpha(q) \ldots$ and $\ldots q, \alpha(p) \ldots$ may belong to the same cycle. Algebraically, this condition comes from $\omega=\alpha W$ in (2.46). If we write $W \ni(p q)$, then the equation (B.7) implies

$$
\omega \ni(\ldots p, \alpha W(p) \ldots) \quad \Leftrightarrow \quad \omega \ni(\ldots q, \alpha W(q) \ldots) .
$$

These two sequences produce the same flavor factor $\sqrt{h^{A_{p} A_{q}}}$. Thus, the consistency condition (B.7) or (B.8) is equivalent to the square-root-free condition; namely, the total product $\prod_{R=1}^{C(\omega)} \mathbb{F} \cdot \Phi_{R}$ in (2.47) does not have the square root of $h$ 's.

Based on the above arguments, we conjecture that the $n$-point function is given by the sum over $\omega \in \mathcal{W}(w)$ which does not contain $\sqrt{h}$ 's:

$$
G_{n}=\left.\sum_{\substack{w \vdash 2 L \\ w_{1}=0}} \prod_{f} \frac{1}{w_{f} ! f^{w_{f}}} \sum_{\gamma \in S_{2 L}} \mathbb{F}\left(\gamma \omega_{0} \gamma^{-1}\right)\right|_{\sqrt{h} \rightarrow 0} .
$$

We checked that this equation is consistent with the original $S_{2 L}$ formula (2.18), by computing some simple cases by Mathematica.

\section{Vertex-based skeleton formula}

We express $G_{n}$ by gluing open two-points, following the vertex-based method in section 2.1.

\section{C.1 Gluing open two-point}

Let us unify the color indices of $\left\{\mathcal{O}_{i}\right\}$ in $(4.20)$ as

$$
P \in\{1,2, \ldots, 2 L\}=\left\{(\mathrm{P}, \bar{p}) \mid \bar{p} \in\left\{1,2, \ldots, \ell_{\mathrm{P}}\right\}, \mathrm{P} \in\{1,2, \ldots, 2 \bar{L}\}\right\} .
$$

We also unify the reduced permutations and the reduced Wick-contractions as

$$
\bar{\alpha}=\prod_{i=1}^{n} \bar{\alpha}_{i} \in S_{\bar{L}}, \quad V=\prod_{\mathrm{P}=1}^{\bar{L}}\left(\bar{\gamma}\left(a_{2 \mathrm{P}-1}\right) \bar{\gamma}\left(a_{2 \mathrm{P}}\right)\right) \in \mathbb{Z}_{2}^{\otimes \bar{L}}, \quad\left(\bar{\gamma} \in S_{2 \bar{L}}\right)
$$



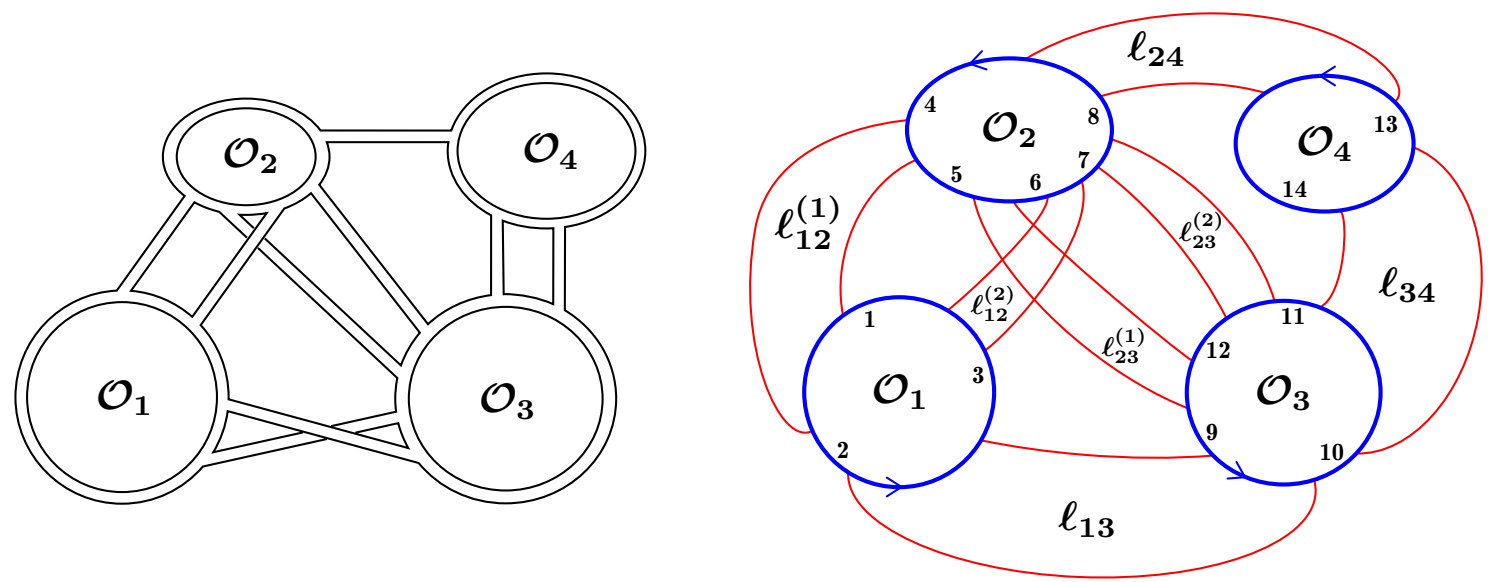

Figure 21. (Left) Example of a set of the Wick-contractions in $\left\langle\mathcal{O}_{1} \mathcal{O}_{2} \mathcal{O}_{3} \mathcal{O}_{4}\right\rangle$ with $\left(L_{1}, L_{2}, L_{3}, L_{4}\right)=(4,5,6,3)$. (Right) Skeleton-reduced graph for the same set of Wickcontractions, whose edges are colored as in figure 1 . This corresponds to $V=$ $(1,4)_{1}(2,9)_{2}(3,6)_{1}(5,12)_{1}(7,11)_{1}(8,13)_{1}(10,14)_{2}$ with $\bar{L}=7$, where the subscript in $V$ represents the bridge length of each open two-point.

just like (2.7). The Cayley graph of $(V, \bar{\alpha})$ is the skeleton-reduced Feynman graph, and a positive integer $\ell_{E}$ is associated to the $E$-th edge. Figure 21 shows an example of the skeleton reduction applied to a Feynman graph in a four-point function.

In the new notation, the bridge lengths $\left\{\ell_{i j}^{(\rho)}\right\}$ in (4.15) simply become $\left\{\ell_{\mathrm{P}}\right\}$, and the number of non-zero $\ell_{i j}^{(\rho)}$ 's is equal to $\bar{L}$,

$$
\sum_{j \neq i} r_{i j}=\bar{L}_{i}, \quad \sum_{i<j} r_{i j}=\bar{L}, \quad \bar{L}=\frac{1}{2} \sum_{i} \bar{L}_{i} .
$$

Note that the range of $\mathrm{P}$ is twice as big as that of $(i j, \rho)$. An open two-point function has four endpoints, labeled by P. Using the global orientation, we may use two of the four endpoints to specify the same open two-point. It means that

$$
\tau_{\mathrm{P}}=\tau_{\mathrm{Q}} \quad \Leftrightarrow \quad\left(a_{\mathrm{P}} a_{\mathrm{Q}}\right) \in V .
$$

The same remark applies to the color $R$-matrix, which has the symmetry $R_{a^{\prime} b^{\prime}}^{a b}=R_{b^{\prime} a^{\prime}}^{b a}$.

Let us rewrite $G_{n}$ in (4.24) as $^{31}$

$$
\begin{aligned}
& G_{n}=\sum_{\left\{\text {partition of } \mathcal{O}_{i}\right\}} \sum_{V: \text { external }} \sum_{\tau: \text { internal }} \prod_{\mathrm{P}=1}^{2 \bar{L}}\left[\left(\prod_{\bar{p}=1}^{\ell_{\mathrm{P}}} h^{A_{\mathrm{P}, \bar{p}} A_{V(\mathrm{P}), \tau_{\mathrm{P}}(\bar{p})}}\right) N_{c}^{\bar{Z}\left(\tau_{\mathrm{P}}\right)} R_{a_{\mathrm{P}+\ell_{\mathrm{P}}} a_{V(\mathrm{P})+\ell_{\mathrm{P}}}}^{a_{\mathrm{P}} a_{V(\mathrm{P})}}\right]^{1 / 2} \\
& =\sum_{\{\text {partition of }} \sum_{\left.\mathcal{O}_{i}\right\}} \sum_{V \text { : external }} \sum_{\tau: \text { internal }} N_{c}^{C(\bar{\omega})+\frac{1}{2} \sum_{\mathrm{P}} \bar{Z}\left(\tau_{\mathrm{P}}\right)} \mathfrak{H}(W)
\end{aligned}
$$

\footnotetext{
${ }^{31}$ By abuse of notation, we use $\left(a_{\mathrm{P}} a_{\mathrm{Q}}\right)$ and $(\mathrm{PQ})$ interchangeably, like $\left(a_{\mathrm{P}} a_{\mathrm{Q}}\right) \in V$ or $(\mathrm{PQ}) \in V$. Recall that originally $a_{\mathrm{P}}$ is the color index running from 1 to $N_{c}$ for the P-th vertex.
} 
where we introduced new symbols

$$
\begin{aligned}
\mathfrak{H}(W) & \equiv\left(\prod_{\mathrm{P}=1}^{2 \bar{L}} \prod_{\bar{p}=1}^{\ell_{\mathrm{P}}} h^{A_{\mathrm{P}, \bar{p}} A_{V(\mathrm{P}), \tau_{\mathrm{P}}(\bar{p})}}\right)^{1 / 2}, \\
N_{c}^{C(\bar{\omega})} & \equiv\left(\prod_{\mathrm{P}=1}^{2 \bar{L}} \prod_{\bar{p}=1}^{\ell_{\mathrm{P}}} R_{\left.a_{\mathrm{P}+\ell_{\mathrm{P}}} a_{V(\mathrm{P})+\ell_{\mathrm{P}}} a^{a_{2}}\right)^{1 / 2}, \quad\left(\bar{\omega} \in S_{2 \bar{L}}\right) .}\right.
\end{aligned}
$$

We can think of $\bar{\omega}$ as a face permutation, because $R$ is a pair of $\delta$-functions as discussed in (4.8). The equation (C.8) makes sense because r.h.s. has $4 \bar{L}$ color indices, and the same index always appears as a superscript and a subscript, once for each. Furthermore, by comparing (2.10) and (C.6), we find

$$
C(\alpha W)=C(\bar{\omega})+\frac{1}{2} \sum_{\mathrm{P}} \bar{Z}\left(\tau_{\mathrm{P}}\right)=C(\bar{\omega})+\sum_{i<j} \sum_{\rho} Z\left(\alpha_{i} W_{i j, \rho} \alpha_{j} W_{i j, \rho}^{-1} \mid\{\text { end-points }\}\right)
$$

where we used (4.11). This means that $\bar{Z}$ counts the number of cycles without open end-points, and $C(\bar{\omega})$ counts those with open end-points.

\section{C.2 Face permutations of the skeleton graph}

There exist two face permutations for the skeleton graph. The first one is given by

$$
\nu \equiv \bar{\alpha} V \in S_{2 \bar{L}}
$$

which generates the faces of the skeleton graph. Since the skeleton graph triangulates a Riemann surface, $\nu$ does not have one- and two-cycles. The second one is $\bar{\omega} \in S_{2 \bar{L}}$ introduced in (C.8), which counts the color-index cycles and follows from the skeleton reduction of the original face permutation,

$$
\text { Reduction : } \omega \mapsto \bar{\omega} \text {. }
$$

If we consider the ladder-skeleton reduction (assembling only planar ladders), $\nu$ and $\bar{\omega}$ are identical.

The two face permutations $\nu$ and $\bar{\omega}$ agree if the matrix $R_{a^{\prime} b^{\prime}}^{a b}$ in (4.5) takes the planar form, $R_{a^{\prime} b^{\prime}}^{a b}=\delta_{b^{\prime}}^{a} \delta_{a^{\prime}}^{b}$. For non-planar cases, we can define another involution $\theta \in \mathbb{Z}_{2}^{\bar{L}}$ such that

$$
\nu \equiv \bar{\omega} \theta, \quad \theta^{2}=1
$$

This $\theta$ also follows from $\tau$ in (4.4) thanks to the selection rule (4.8). An example of the relation (C.12) is illustrated in figure 22.

\section{C.3 Space of the reduced Wick-contractions}

Our next goal is to construct the space of the reduced Wick-contractions in $G_{n}$, namely the range of three sums in (C.6). 

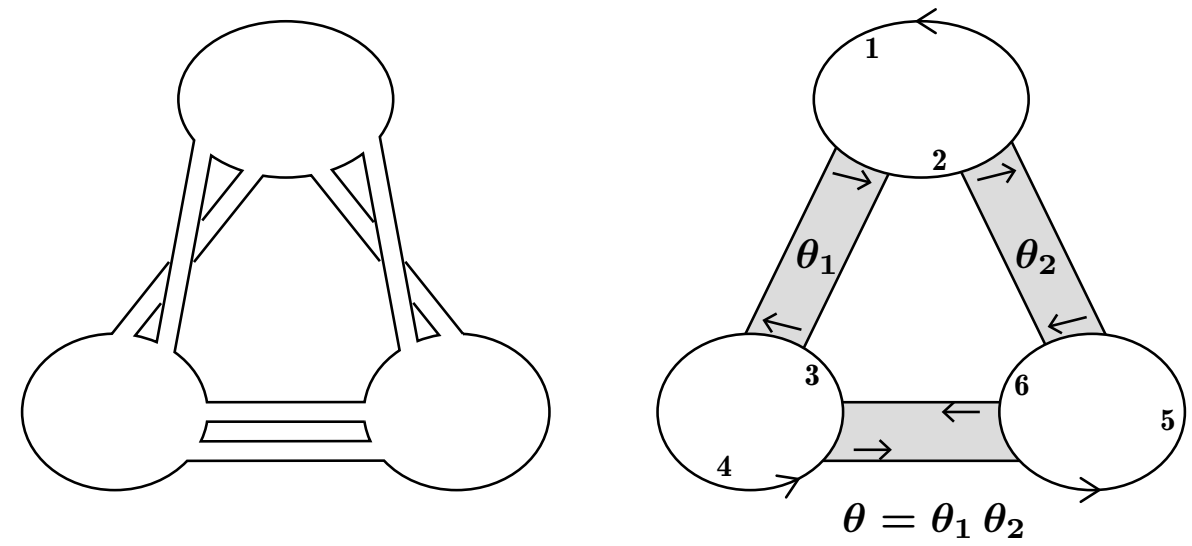

Figure 22. (Left) Original Feynman graph, (Right) Skeleton-reduced graph with $\bar{\alpha}=(12)(34)(56)$ and $V=(13)(25)(46)$. The two face permutations of the skeleton graph are $\nu=(145)(263)$ which neglects the internal Wick-contractions, and $\bar{\omega}=(12)(3456)$ which remembers the internal structure through $\theta=(13)(25)$.

\section{C.3.1 Partitions of an operator}

We generate partitions of the operator $\mathcal{O}_{i}$ in (4.19) as follows: choose an ordered partition of $L_{i}$, divide $\mathcal{O}_{i}$ according to that partition, and apply $\mathbb{Z}_{L_{i}}$ translations. An ordered partition of $L_{i}$ is denoted by

$$
\ell_{i} \equiv\left(\ell_{i \mid 1}, \ell_{i \mid 2}, \ldots, \ell_{i \mid \bar{L}_{i}}\right) \vdash L_{i}, \quad L_{i}=\sum_{q=1}^{\bar{L}_{i}} \ell_{i \mid q}
$$

We group the sequences of $\boldsymbol{\ell}_{i \mid q}$ consecutive fields together as in (4.2) to define the reduced operator

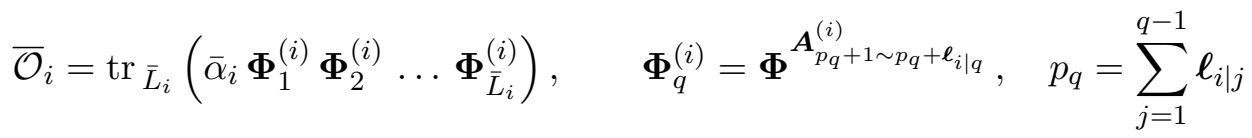

with $\bar{\alpha}_{i} \in S_{\bar{L}_{i}}$.

For simplicity, we assume that $\bar{\alpha}_{i} \in \mathbb{Z}_{\bar{L}_{i}}$ and $\overline{\mathcal{O}}_{i}$ are single-traces. We should identify the cyclically translated operators as long as the two ordered partitions are equal,

$$
\begin{aligned}
\operatorname{tr}\left(\boldsymbol{\Phi}_{1}^{(i)} \boldsymbol{\Phi}_{2}^{(i)} \ldots \boldsymbol{\Phi}_{\bar{L}_{i}}^{(i)}\right) & \sim \operatorname{tr}\left(\boldsymbol{\Phi}_{m+1}^{(i)} \ldots \boldsymbol{\Phi}_{\bar{L}_{i}}^{(i)} \boldsymbol{\Phi}_{1}^{(i)} \ldots \boldsymbol{\Phi}_{m}^{(i)}\right) \\
& \Leftrightarrow\left(\boldsymbol{\ell}_{i \mid 1}, \boldsymbol{\ell}_{i \mid 2}, \ldots, \boldsymbol{\ell}_{i \mid \bar{L}_{i}}\right)=\left(\boldsymbol{\ell}_{i \mid m+1}, \ldots, \boldsymbol{\ell}_{i \mid \bar{L}_{i}}, \boldsymbol{\ell}_{i \mid 1}, \ldots, \boldsymbol{\ell}_{i \mid m}\right) .
\end{aligned}
$$

This identification becomes important when $\boldsymbol{\ell}_{i}$ has a repeated pattern. ${ }^{32}$ The original $\mathbb{Z}_{L_{i}}$ symmetry generates inequivalent reduced operators, unless the equivalence relation (C.15)

\footnotetext{
${ }^{32}$ For example, if $\boldsymbol{\ell}_{i}=(3,2,3,2)$, the cyclic translation $\Phi^{A_{p}^{(i)}} \mapsto \Phi^{A_{p+5}^{(i)}}$ generates the same reduced operator, which corresponds to the identity $\operatorname{tr}\left(\boldsymbol{\Phi}_{1}^{(i)} \boldsymbol{\Phi}_{2}^{(i)} \boldsymbol{\Phi}_{3}^{(i)} \boldsymbol{\Phi}_{4}^{(i)}\right)=\operatorname{tr}\left(\boldsymbol{\Phi}_{3}^{(i)} \boldsymbol{\Phi}_{4}^{(i)} \boldsymbol{\Phi}_{1}^{(i)} \boldsymbol{\Phi}_{2}^{(i)}\right)$.
} 
applies. For example, the following two reduced operators are inequivalent:

$$
\begin{aligned}
\operatorname{tr}\left(\left(\Phi^{A_{1}} \Phi^{A_{2}}\right)\left(\Phi^{A_{3}} \Phi^{A_{4}}\right)\right) & \sim \operatorname{tr}\left(\left(\Phi^{A_{3}} \Phi^{A_{4}}\right)\left(\Phi^{A_{1}} \Phi^{A_{2}}\right)\right) \\
& \nsim \operatorname{tr}\left(\left(\Phi^{A_{2}} \Phi^{A_{3}}\right)\left(\Phi^{A_{4}} \Phi^{A_{1}}\right)\right) \sim \operatorname{tr}\left(\left(\Phi^{A_{4}} \Phi^{A_{1}}\right)\left(\Phi^{A_{2}} \Phi^{A_{3}}\right)\right) .
\end{aligned}
$$

We introduce an ordered set of $L_{i}$ numbers $\varpi_{i}$ as a function of $z \in \mathbb{Z}_{L_{i}}$,

$$
\begin{aligned}
\varpi_{i}(z) \equiv\left\{z(1), z(2), \ldots, z\left(\boldsymbol{\ell}_{i \mid 1}\right)\right\}\{ & \left.z\left(p_{2}+1\right), z\left(p_{2}+2\right), \ldots, z\left(p_{2}+\boldsymbol{\ell}_{i \mid 2}\right)\right\} \ldots \\
& \left\{z\left(p_{\bar{L}_{i}}+1\right), z\left(p_{\bar{L}_{i}}+2\right), \ldots, z\left(p_{\bar{L}_{i}}+\boldsymbol{\ell}_{i \mid \bar{L}_{i}}\right)\right\}
\end{aligned}
$$

and denote its equivalence class by $\left[\varpi_{i}(z)\right]$ modulo the relation (C.15). In general, the number of equivalence classes is given by the orbit-stabilizer theorem, which means

$$
\begin{aligned}
\left(\text { Number of }\left[\varpi_{i}(z)\right]\right) & =\frac{\left|\mathbb{Z}_{L_{i}}\right|}{\left|\operatorname{Stab}_{\mathbb{Z}_{L_{i}}}\left(\varpi_{i}\left(z_{0}\right)\right)\right|} \\
\operatorname{Stab}_{\mathbb{Z}_{L_{i}}}\left(\varpi_{i}\left(z_{0}\right)\right) & =\left\{z \in \mathbb{Z}_{L_{i}} \mid \varpi_{i}(z) \sim \varpi_{i}\left(z^{\prime}\right) \text { as in }(\text { C.15) }\}\right.
\end{aligned}
$$

where $z_{0}$ is a reference point, e.g. identity. In summary, the space of the partitions of $\mathcal{O}_{i}$ can be written as

$$
\begin{aligned}
\left(\text { Partition of } \mathcal{O}_{i}\right)= & \left\{\text { Choice of an ordered list } \ell_{i} \vdash L_{i}\right\} \\
& \times\left\{\text { Choice of an inequivalent cyclic translation }\left[\varpi_{i}(z)\right] \mid z \in \mathbb{Z}_{L_{i}}\right\} .
\end{aligned}
$$

\section{C.3.2 Space of internal Wick-contractions}

Let us introduce a new partition of $L$ by ${ }^{33}$

$$
[\ell]=\left[1^{\boldsymbol{r}_{1}} 2^{\boldsymbol{r}_{2}} \ldots\right] \vdash L, \quad C([\ell])=\sum_{k} \boldsymbol{r}_{k}=\bar{L}
$$

where $\boldsymbol{r}_{k}$ is the number of consecutive Wick-contractions with $k$ fields. This partition is related to $\boldsymbol{\ell}_{i}=\left\{\boldsymbol{\ell}_{i \mid q}\right\}$ in (C.13) by

$$
\bigsqcup_{i=1}^{n} \boldsymbol{\ell}_{i}=\left[1^{2 \boldsymbol{r}_{1}} 2^{2 \boldsymbol{r}_{2}} \ldots\right] \vdash 2 L .
$$

This relation is exemplified in figure 23. For each open two-point, we can define the internal Wick-contractions $\tau_{E} \in S_{\ell_{E}}$ by (4.4). The union of $\left\{\tau_{E}\right\}$ gives

$$
\boldsymbol{\tau} \equiv\left(\tau_{1}, \tau_{2}, \ldots, \tau_{\bar{L}}\right) \in S_{\boldsymbol{\tau}} \equiv \bigotimes_{k \geq 1} S_{k}^{\otimes \boldsymbol{r}_{k}}
$$

\footnotetext{
${ }^{33}$ This $[\ell]$ is no longer an ordered set.
} 


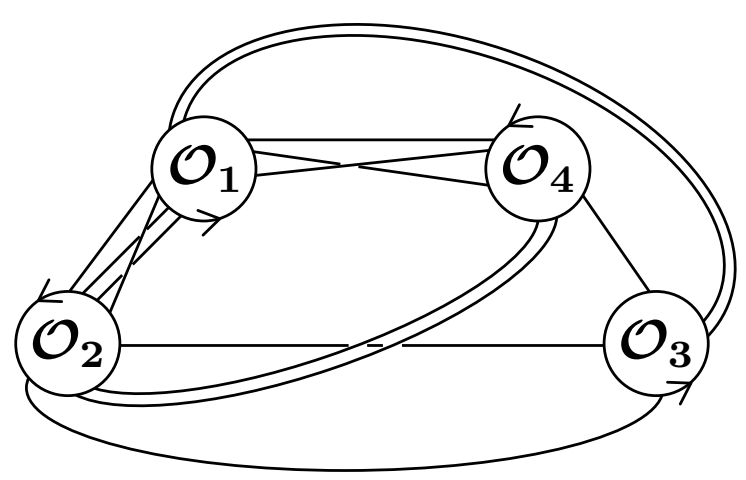

Figure 23. This diagram corresponds to $[\ell]=\left[1^{3} 2^{2} 3^{1} 4^{1}\right], L=14$ and $\bar{L}=7$. The individual partitions of lengths are $\boldsymbol{\ell}_{1}=(4,3,2), \boldsymbol{\ell}_{2}=(4,1,2,1), \boldsymbol{\ell}_{3}=(2,1,1,1)$ and $\boldsymbol{\ell}_{4}=(3,2,1)$, given in the counterclockwise order.

\section{C.3.3 Space of external Wick-contractions}

One definition of this space has already been given in (C.2) as

$$
V \in\left\{\prod_{\mathrm{P}=1}^{\bar{L}}\left(\bar{\gamma}\left(a_{2 \mathrm{P}-1}\right) \bar{\gamma}\left(a_{2 \mathrm{P}}\right)\right) \mid \bar{\gamma} \in S_{2 \bar{L}} / S_{\bar{L}}\left[\mathbb{Z}_{2}\right]\right\} .
$$

We restrict this space, based on a given set of partitions $\left\{\overline{\mathcal{O}}_{i}\right\}$. The open two-point is non-zero only if the pair of the sequential fields have the same length (4.3). We take the external Wick-contractions over all possible pairs of the same length. Let us write $V=\left(V_{1}, V_{2}, \ldots\right) \in \mathbb{Z}_{2}^{\otimes \bar{L}}$ where $V_{k} \in \mathbb{Z}_{2}^{\otimes \boldsymbol{r}_{k}}$ is the external Wick-contractions of length $k$. We sum $V_{k}$ over the space

$$
V_{k} \in \mathcal{V}_{k}=\left\{\prod_{j=1}^{\boldsymbol{r}_{k}}\left(g_{k}\left(a_{2 j-1}^{(k)}\right) g_{k}\left(a_{2 j}^{(k)}\right)\right) \mid g_{k} \in S_{2 \boldsymbol{r}_{k}} / S_{\boldsymbol{r}_{k}}\left[\mathbb{Z}_{2}\right]\right\} .
$$

The restricted space of external Wick-contractions is defined as the union of $\left\{\mathcal{V}_{k}\right\}$,

$$
V \in \mathcal{V} \equiv\left(\mathcal{V}_{1}, \mathcal{V}_{2}, \ldots, \mathcal{V}_{\bar{L}}\right)
$$

This definition of $\mathcal{V}$ still contains two types of unphysical Wick-contractions. The first type is self-contractions, which can be removed by $h^{A B}$. The second type is mutually adjacent pairs of open two-points. As discussed at the beginning of this section, a skeleton graph should not have faces made of two or fewer edges. As a result, the symbol $\boldsymbol{\Phi}_{p}^{(i)}$ represents the maximal sequence of consecutive fields appearing in the original Wick-contractions. This condition is equivalent to the exclusion of taking external Wick-contractions from the mutually adjacent pairs. For example, when $\left(\boldsymbol{\Phi}_{p}^{(i)}, \boldsymbol{\Phi}_{q}^{(j)}\right)$ are contracted, then the four possibilities $\left(\boldsymbol{\Phi}_{p \pm 1}^{(i)}, \boldsymbol{\Phi}_{q \pm 1}^{(j)}\right)$ are not allowed. We define $\mathcal{V}_{\text {phys }}$ as the subspace of $\mathcal{V}$ in (C.25) having no mutually adjacent pairs. A necessary but not sufficient condition for getting $\mathcal{V}_{\text {phys }} \subset \mathcal{V}$ is to require that the faces have no two-cycles, ${ }^{34}$

$$
\mathcal{V}_{\text {phys }} \subsetneq\{V \in \mathcal{V} \mid C(\bar{\alpha} V)=C(\bar{\alpha} V \bar{\alpha} V)\} .
$$

\footnotetext{
${ }^{34}$ The one-cycles in $\bar{\alpha} V$ were removed by $h^{A B}$.
} 
r.h.s. does not have adjacent ladder-type skeleton edges, but still contains adjacent skeleton edges which mutually cross and do not form a two-cycle. We need to throw away external Wick-contractions of this type.

\section{C.3.4 Rewriting the $n$-point functions}

As discussed in section 4.2, there is a bijection between the original Wick-contraction and $W$ and the triple $\left(\left\{\overline{\mathcal{O}}_{i}\right\}, V, \boldsymbol{\tau}\right)$. The spaces (C.19), (C.26) and (C.22) offer an alternative definition of the space of (physical) Wick-contractions given in (2.17). We write the $n$-point functions (C.6) as

$$
G_{n}^{\prime}=\sum_{\left\{\text {partition of } \mathcal{O}_{i}\right\}} \sum_{V \in \mathcal{V}_{\text {phys }}} \sum_{\boldsymbol{\tau} \in S_{\boldsymbol{\tau}}} N_{c}^{\bar{Z}(\boldsymbol{\tau})+C(\bar{\omega})} \mathfrak{H}(W)
$$

where

$$
\bar{Z}(\boldsymbol{\tau}) \equiv \frac{1}{2} \sum_{\mathrm{P}=1}^{2 \bar{L}} \bar{Z}\left(\tau_{\mathrm{P}}\right)=\sum_{E=1}^{\bar{L}} \bar{Z}\left(\tau_{E}\right) .
$$

The symbol $G_{n}^{\prime}$ in (C.27) means that the disconnected parts involving a two-point function are removed. This is because the skeleton reduction of a two-point function gives a face permutation consisting of a two-cycle. We excluded such terms in (C.26).

Let us compare the vertex-based skeleton formula (C.27) and the face-based one (4.50). In the vertex-based formula, we partition the operators $\left\{\mathcal{O}_{i}\right\}$ and apply the Wick contractions of open two-point functions. Throughout this process, we keep track the flavor indices of $\left\{\mathcal{O}_{i}\right\}$ and avoided the over-counting of the reduced operator as in (C.15). In contrast, in the face-based formula we sum over the skeleton-graph topologies by using face permutations. The flavor indices of $\mathcal{O}_{i}$ are restored only in the end (4.49), and we can sum over the orbits of cyclic translations without bothering about the over-counting.

One may argue that the face-based formula is simpler than the vertex-based one, because the former contains more fictitious degrees of freedom. Roughly speaking, the sum $V \in \mathcal{V}_{\text {phys }}$ in (C.27) should be smaller than $\nu \in\left(S_{2 \bar{L}}^{\times \times} / \text {Aut } V\right)_{\text {phys }}$ in (4.50), because $\mathcal{V}_{\text {phys }} \subset \prod_{k} S_{2 r_{k}} \subset S_{2 \bar{L}}$.

\section{More on geometry and graphs}

\section{D.1 Moduli space}

In the literature there are various definitions of the moduli space of Riemann surfaces denoted by $\mathcal{M}_{g, n}$;

- Space of the complex structure on $\Sigma_{g, n}$

- Space of the conformal class of Riemannian metric $g \sim e^{2 \rho} g$ on $\Sigma_{g, n}$

- Space of hyperbolic metrics modulo mapping class groups for $g \geq 2$ [73]

- Space of smooth or stable curves up to isomorphism [74]

- $\cdots$ 
The moduli space $\mathcal{M}_{g, n}$ has the dimensions

$$
\operatorname{dim}_{\mathbb{C}} \mathcal{M}_{g, n}=n-3+3 g
$$

which is parametrized locally by

$$
\mathcal{M}_{g, n} \simeq \begin{cases}\left\{z_{4}, \ldots, z_{n}\right\} & (g=0) \\ \left\{z_{2}, \ldots, z_{n}\right\} \cup\{\tau\} & (g=1) \\ \left\{z_{1}, \ldots, z_{n}\right\} \cup\left\{\mu_{1}, \ldots, \mu_{3 g-3}\right\} & (g \geq 2)\end{cases}
$$

One way to identify $\left\{\mu_{a}\right\}$ is to take pants decomposition by cutting a genus $g$ curve $(3 g-3)$ times, and use Fenchel-Nielsen coordinates to fix the moduli [25].

In general, it is not easy to construct the whole $\mathcal{M}_{g, n}$ explicitly, because $\mathcal{M}_{g, n}$ is not compact. When one approaches the boundary of $\mathcal{M}_{g, n}$, the Riemann surface $\Sigma_{g, n}$ degenerates, either by pinching the handle or by colliding punctures. Deligne and Mumford considered compactification of $\mathcal{M}_{g, n}$ by adding boundary components in the language of algebraic geometry [24].

Harer, Mumford and Thurston suggested studying the moduli space of decorated Riemann surfaces $\mathcal{M}_{g, n} \times \mathbb{R}_{+}^{n}$ instead of $\mathcal{M}_{g, n}$ [20]. By decorated we mean that the complex structure is induced by the quadratic differential having the double pole with the specific residue $r_{i} \in \mathbb{R}_{+}$at the puncture $p_{i}$. This idea works if there is a bijection between the complex structure and the quadratic differential on $\Sigma_{g, n}$. This is indeed true for the Jenkins-Strebel quadratic differential discussed in appendix D.3.

\section{D.2 Ribbon graphs}

A graph is defined as a set of vertices and edges, and we usually talk of connected unoriented graphs whose vertices are labeled. A metric ribbon graph is a graph with the following property:

1. Each vertex has valency at least three,

2. Edges connecting to a vertex are cyclically ordered,

3. A positive real number called length is assigned to each edge.

Any graphs drawn on a Riemann surface without intersection can be ribbonized [48]. ${ }^{35}$ For this purpose, we replace vertices by disks, edges by strips, and glue them together while keeping the cyclic ordering.

A topological cell decomposition of $\Sigma_{g, n}$ defines a ribbon graph, by identifying the punctures as the vertices of the graph. The cell decomposition is called complete (or ideal) triangulation if all faces of the graph are triangles. If some faces are squares or higher polygons, then the triangulation is called incomplete.

Consider a graph which completely triangulates $\Sigma_{g, n}$. The number of vertices, edges and faces of the graph is given by

$$
V_{g, n}=2 n-4+4 g, \quad E_{g, n}=3 n-6+6 g, \quad F_{g, n}=n, \quad \chi=V_{g, n}-E_{g, n}+F_{g, n}=2-2 g .
$$

\footnotetext{
${ }^{35} \mathrm{~A}$ ribbon graph is also called a fat graph in the literature.
} 
For example, a planar graph can have at most $3 n-6$ non-zero edges, and a toric (genusone) graph can have $3 n$ non-zero edges. A graph is called complete if any pairs of faces must be connected by a single edge. A complete graph exists when

$$
\left(\begin{array}{l}
n \\
2
\end{array}\right)-(3 n-6+6 g) \leq 0
$$

namely, $n \leq 4$ at $g=0$ and $n \leq 7$ at $g=1$.

Consider the dual ribbon graph $\Gamma_{g, n}$ of an incomplete triangulation of $\Sigma_{g, n}$. We denote the number of $k$-gons in this graph by $d_{k}$, and define the total face degree of this graph by

$$
\operatorname{Deg}\left(\Gamma_{g, n}\right) \equiv \sum_{k \geq 3} d_{k}(k-2) .
$$

It follows that

$$
\operatorname{Deg}\left(\Gamma_{g, n}\right)=2 n-4+4 g
$$

for any complete or incomplete triangulations. This is because incomplete triangulations are generated by gluing or splitting of $a$-gons and $b$-gons

$$
a \oplus b \quad \leftrightarrow \quad(a+k) \oplus(b-k)
$$

and by removing 2-gons. This procedure leaves (D.5) invariant. The incompleteness of the triangulation can be measured by counting the number of edges,

$$
\text { (Number of edges in } \left.\Gamma_{g, n}\right) \leq E_{g, n}=3 n-6+6 g \text {. }
$$

Since an edge has two endpoints, l.h.s. is also equal to the half of the total valency of the vertices in $\Gamma_{g, n}$.

\section{D.3 Quadratic differentials}

A quadratic differential is a meromorphic function which satisfies the transformation rule

$$
\varphi \equiv \phi(z) d z^{2}=\phi(w) d w^{2} .
$$

Given a quadratic differential, one can compute the length and area by

$$
L\left(p_{0}, p_{1}\right)=\int_{p_{0}}^{p_{1}} \sqrt{\varphi}, \quad A(\mathcal{R})=\iint_{\mathcal{R}} d z d \bar{z}|\phi(z)| .
$$

Given a reference point $p_{0}$, the horizontal and vertical trajectories of $\varphi$ are defined by

$$
\begin{aligned}
\text { Horizontal trajectory } & =\left\{z \in \Sigma \mid \operatorname{Im}\left(\int_{p_{0}}^{z} \sqrt{\varphi}\right)=\text { constant }\right\} \\
\text { Vertical trajectory } & =\left\{z \in \Sigma \mid \operatorname{Re}\left(\int_{p_{0}}^{z} \sqrt{\varphi}\right)=\text { constant }\right\} .
\end{aligned}
$$

Below we consider the Jenkins-Strebel (JS) quadratic differential following [47]. The JS differential satisfies a certain minimal-area condition, and it exists uniquely for each punctured Riemann surface $[18,19,75]$. 
Definition. Let $\Sigma_{g, n}$ be a Riemann surface with the punctures at $\left\{p_{1}, p_{2}, \ldots, p_{n}\right\}$. The JS quadratic differential with the residue $\left\{r_{1}, r_{2}, \ldots, r_{n}\right\}$ satisfies the following conditions:

1. $\phi$ is holomorphic on $\Sigma_{g, n} \backslash\left\{p_{i}\right\}$

2. $\phi$ has a double pole at $\left\{p_{i}\right\}$

3. All horizontal trajectories are compact, except for measure zero subsets of $\Sigma_{g, n}$

4. Each compact trajectory surrounds one of the poles, and the residue at $p_{j}$ satisfies

$$
\oint d z \sqrt{\phi}=r_{j} .
$$

The branch of the square root is chosen such that $r_{j}>0$ for all $j .{ }^{36}$

By definition, a JS differential does not have poles of order $>2$. We define

$$
\text { (Order of } \varphi)=\sum_{j \geq 1} j \text { (Number of the } j \text {-th zeroes) }-\sum_{k=1,2} k \text { (Number of the } k \text {-th poles). }
$$

It is known that the order of $\varphi$ for $\Sigma_{g, n}$ is given by $^{37}$

$$
(\text { Order of } \varphi)=4 g-4 \text {. }
$$

In particular, if $\varphi$ has only simple zeroes and double poles,

$$
\left.\mathcal{N}_{\text {zero }}(g, n) \equiv \text { (Number of simple zeroes of } \varphi\right)=-4+4 g+2 n .
$$

For example, $\mathcal{N}_{\text {zero }}(0,2)=0$ and $\mathcal{N}_{\text {zero }}(0,3)=2$.

For a given JS differential $\varphi$, we define its critical graph $\Gamma$ as the collection of all horizontal trajectories. Each zero of $\varphi$ is a vertex of $\Gamma$, and each pole of $\varphi$ is a face of $\Gamma$. Suppose $w$ is a local coordinate around the zero or pole of $\varphi$. From

$$
\int d w \sqrt{w} \sim w^{3 / 2}, \quad \int d w w^{-1} \sim \log w
$$

we find that a simple zero of $\varphi$ gives a trivalent vertex of $\Gamma$, while a face of $\Gamma$ can be surrounded by any number of edges.

The critical graph $\Gamma$ has the structure of metric ribbon graphs. $\Gamma$ is a ribbon graph because the graph is drawn on an oriented Riemann surface, and $\Gamma$ is a metric graph because we measure the length of the edges by (D.10). The structure of $\Gamma$ as a metric ribbon graph is fixed by $\varphi$, which implies that a metric ribbon graph specifies a point of the moduli space $\mathcal{M}_{g, n}$. The metric ribbon graphs classify other structures as well, such as the (decorated) Teichmüller space [76] and the projective structure [77].

Example. The JS differential of $\Sigma_{0,3}$ takes the form

$$
\phi(z) d z^{2}=-\frac{1}{4 \pi^{2}}\left\{\frac{r_{0}^{2}}{z^{2}}+\frac{r_{1}^{2}}{(z-1)^{2}}+\frac{r_{0}^{2}+r_{1}^{2}-r_{\infty}^{2}}{z(z-1)}\right\} .
$$

\footnotetext{
${ }^{36}$ The indifference to the sign of residue is one of the reasons why "quadratic" differential is used.

${ }^{37}$ The order of a quadratic differential is twice of that of a linear differential, whose order is $2 g-2$ according to the Riemann-Hurwitz theorem.
} 

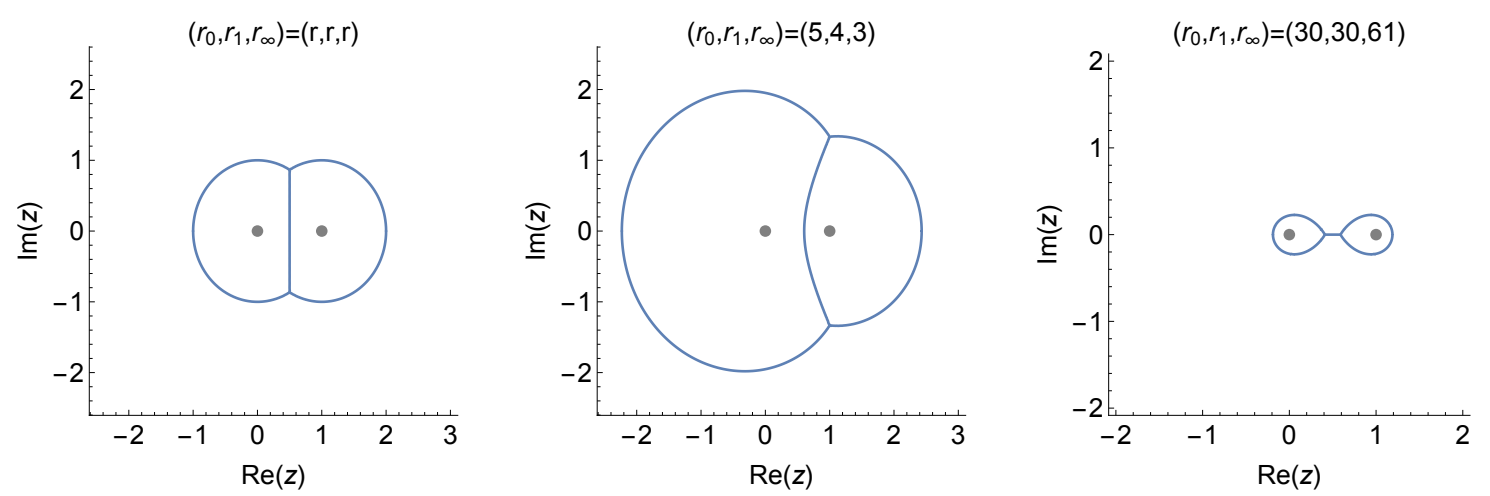

Figure 24. Horizontal trajectories of the JS differential of $\Sigma_{0,3}$ in (D.17). Three poles are at $z=0,1, \infty$, denoted by gray dots. Two zeroes are at $z=\frac{1}{2} \pm i \frac{\sqrt{3}}{2}$ for the left figure, $z=1 \pm i \frac{4}{3}$ for the middle, and $z=\frac{1}{2} \pm \frac{11}{122}$ for the right.

Figure 24 shows the horizontal trajectories for some $\left(r_{0}, r_{1}, r_{\infty}\right)$. When $\left(r_{0}, r_{1}, r_{\infty}\right)$ are not identical, the distance between two zeroes (trivalent vertices) depends on the path one chooses. In particular, there must be a branch cut somewhere on $\Sigma_{g, n}$.

Critical graphs may have self-edges as shown in figure 24. If we denote the length of the edges between zeroes of $\phi$ on $\Sigma_{0,3}$ as $\left(\ell_{1}, \ell_{2}, \ell_{3}\right)$, then the self-edges show up if three edges do not satisfy the triangular inequality $\ell_{i}+\ell_{j}>\ell_{k}$ for any $(i, j, k)$.

Some examples of the JS differential of genus-one surfaces are given in [22]. In general, we need to solve transcendental equations to find the explicit JS differential of given lengths [78].

\section{D.4 Drawing bipartite ribbon graphs}

According to (5.3), any graph on $\Sigma_{g, n}$ (or ideal triangulation of $\Sigma_{g, n}$ ) has a fixed number of vertices, edges and faces when all vertices are trivalent. From such a graph, we derive a bipartite graph, by drawing a white circle for the face and a black dot for the vertex of the original graph. The white circles and black dots should correspond to the double poles and simple zeroes of the Jenkins-Strebel differential on $\Sigma_{g, n}$.

Consider examples of the ribbon graph obtained by a triangulation of Riemann surface. Planar bipartite graphs are shown in figures 25-26, and non-planar graphs in figures 27-28.

Figure 27 shows the bipartite graph from a triangulation of $\Sigma_{1,3}$. This is a toric graph with periodic boundary conditions in both horizontal and vertical directions. The fundamental region of the bipartite graph contains 6 black dots, 9 edges and 3 white circles, in agreement with (5.3). Other examples were given in figure 15. These graphs have the shape of a honeycomb lattice. We can draw similar toric honeycomb lattices for general $n$, satisfying the constraints (5.3).

Figure 28 shows the bipartite graph from $\Sigma_{1,4}$. The fundamental region contains 8 black dots, 12 edges and 4 white circles. Let us label the punctures of this graph. From 

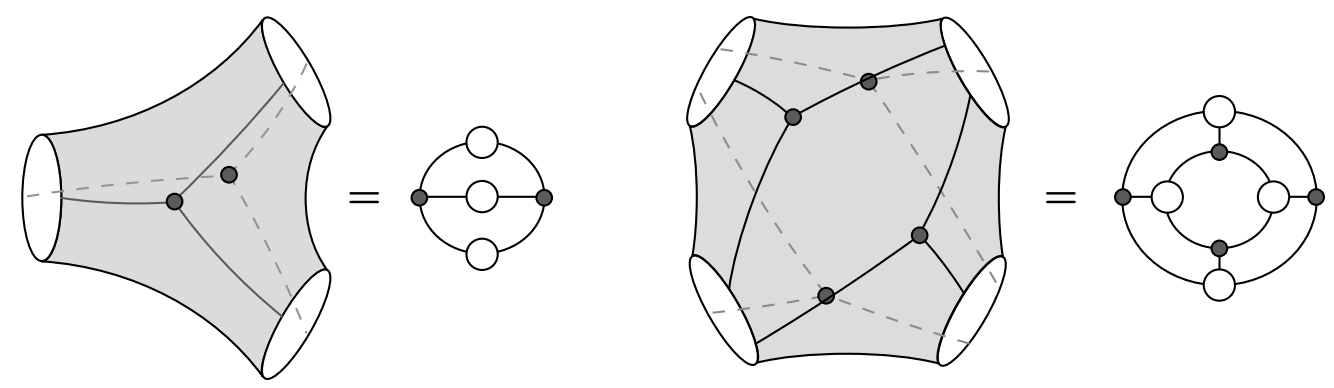

Figure 25. (Metric) ribbon graph from a triangulation of $\Sigma_{0,3}$ and $\Sigma_{0,4}$.
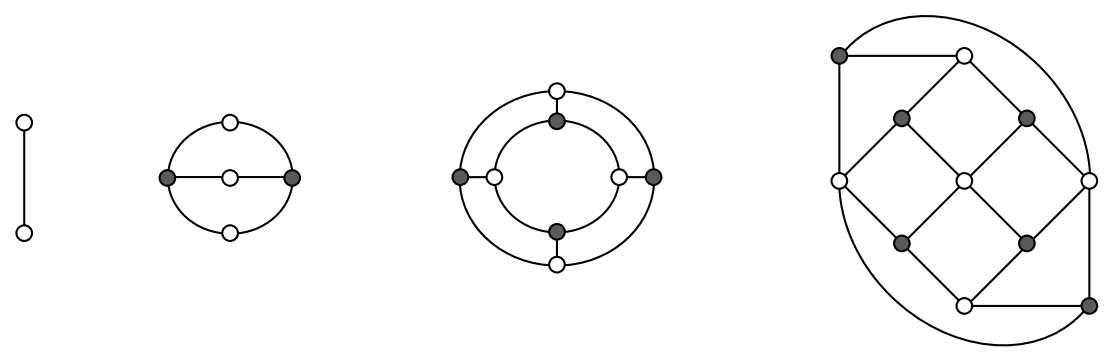

Figure 26. Examples of the planar bipartite graphs for $n=2,3,4,5$.
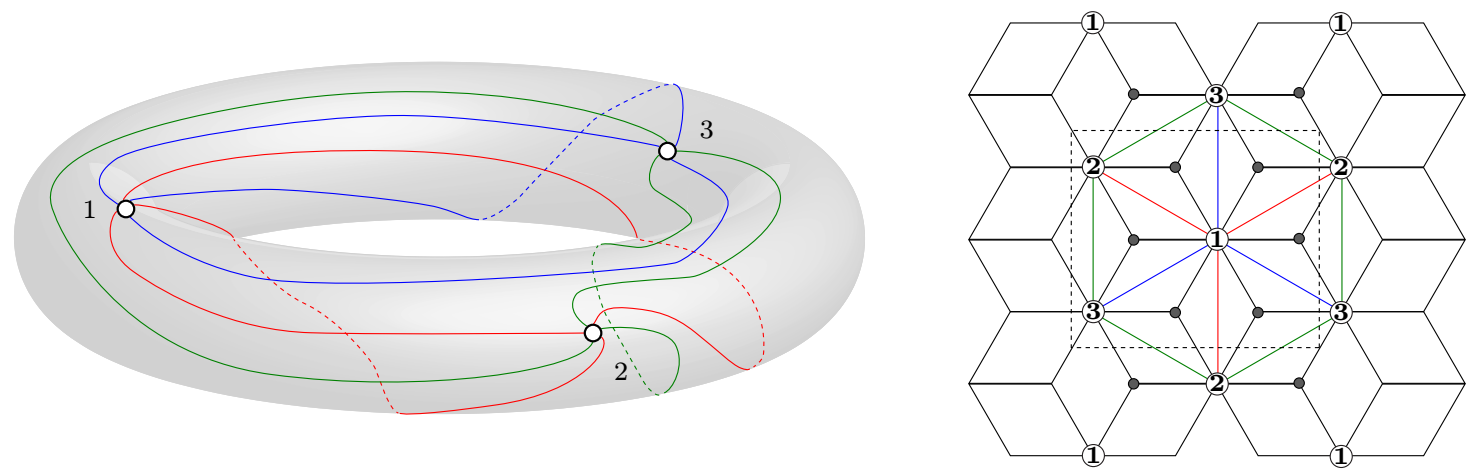

Figure 27. (Left) Triangulation of $\Sigma_{1,3}$. Red, green, blue edges represent $\ell_{12}^{(\rho)}, \ell_{23}^{(\rho)}, \ell_{31}^{(\rho)}$ with $\rho=1,2,3$, respectively. (Right) The same geometry as a toric bipartite graph. The fundamental region is denoted by the dotted square.

the periodicity on the torus, we must have

$$
(a, b, c, d)=\{(2,3,4,1),(3,4,1,2),(4,1,2,3),(1,2,3,4)\} .
$$

Only the first two choices are allowed to avoid self-contractions. When we fix $(a, b, c, d)$, then the periodicity determines $(A, B, C, D)$ uniquely.

Beware that the case of $(g, n)=(1,2)$ is singular. The equation (5.3) says that we should have four trivalent vertices. However, we have only "bivalent" vertices because there are only two punctures. One solution is to introduce fictitious punctures [7]. Another solution is to use two quadrivalent vertices. 


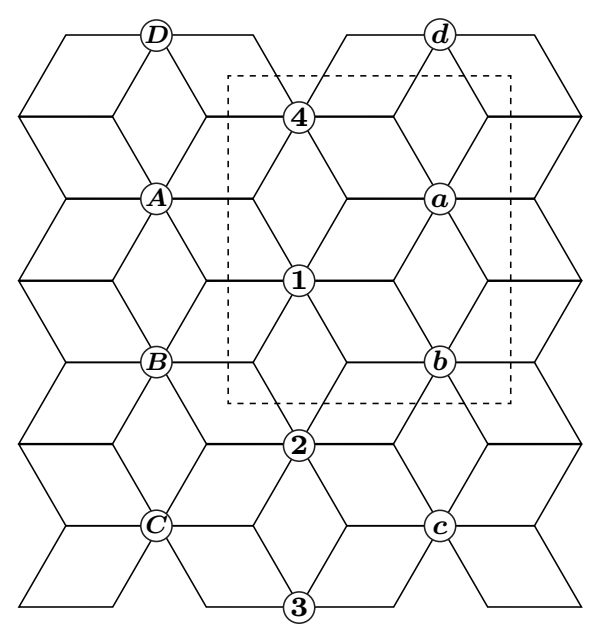

Figure 28. Example of the bipartite graph from $\Sigma_{1,4}$. The dotted square is the fundamental region.

\section{D.5 Feynman graphs on a Riemann surface}

Given a bipartite graph in appendix D.4, we can draw two more ribbon graphs, called black and white. The black graphs are defined by adding edges between the pair of black nodes for each square of the bipartite graph, and then removing all white nodes and the edges connected to them. The white graphs are defined by the same procedure, interchanging black and white. The black and white graphs are dual to each other, as shown in figure 29.

The black graph represents the complex structure of a Riemann surface. The white graph represents the Feynman diagram appearing in the connected $n$-point function of single-trace operators. Thus, the critical graph on a Riemann surface is the dual graph of a Feynman diagram of a gauge theory like $\mathcal{N}=4$ SYM. As a Feynman graph, the white circles represent external operators and black dots represent effective three-point interactions. ${ }^{38}$ The equation (5.3) constrains the graph data of a white graph as

$$
V_{g, n}^{\circ}=n, \quad E_{g, n}^{\circ}=3 n-6+6 g, \quad F_{g, n}^{\circ}=2 n-4+4 g .
$$

Recall that any Feynman diagrams in the double-line notation can be drawn on a Riemann surface. ${ }^{39}$ Thus, the equation (D.19) constrains the cycle type of the vertex permutation $\omega$ of a general Feynman diagram. For example, if the graph consists of strips and triangles only, we can write $[\omega]=\left[2^{w_{2}} 3^{w_{3}}\right] \vdash 2 L$ and impose the equations

$$
2 w_{2}+3 w_{3}=2 L, \quad w_{2}+w_{3}=C(\omega), \quad w_{3}=F_{g, n}^{\circ}
$$

where the last equation comes from (D.6). These equations have a unique solution

$$
w_{2}=L-E_{g, n}^{\circ}=L-3 n+6-6 g, \quad C(\omega)=L-V_{g, n}^{\circ}+\chi=L-n+2-2 g .
$$

\footnotetext{
${ }^{38}$ They are not three-point interactions in the $\mathcal{N}=4$ SYM Lagrangian, because we talk of tree-level correlators.

${ }^{39}$ In other words, the skeleton reduction is unimportant if we are interested in $\Sigma_{g, n}$ rather than $\mathcal{M}_{g, n}$.
} 

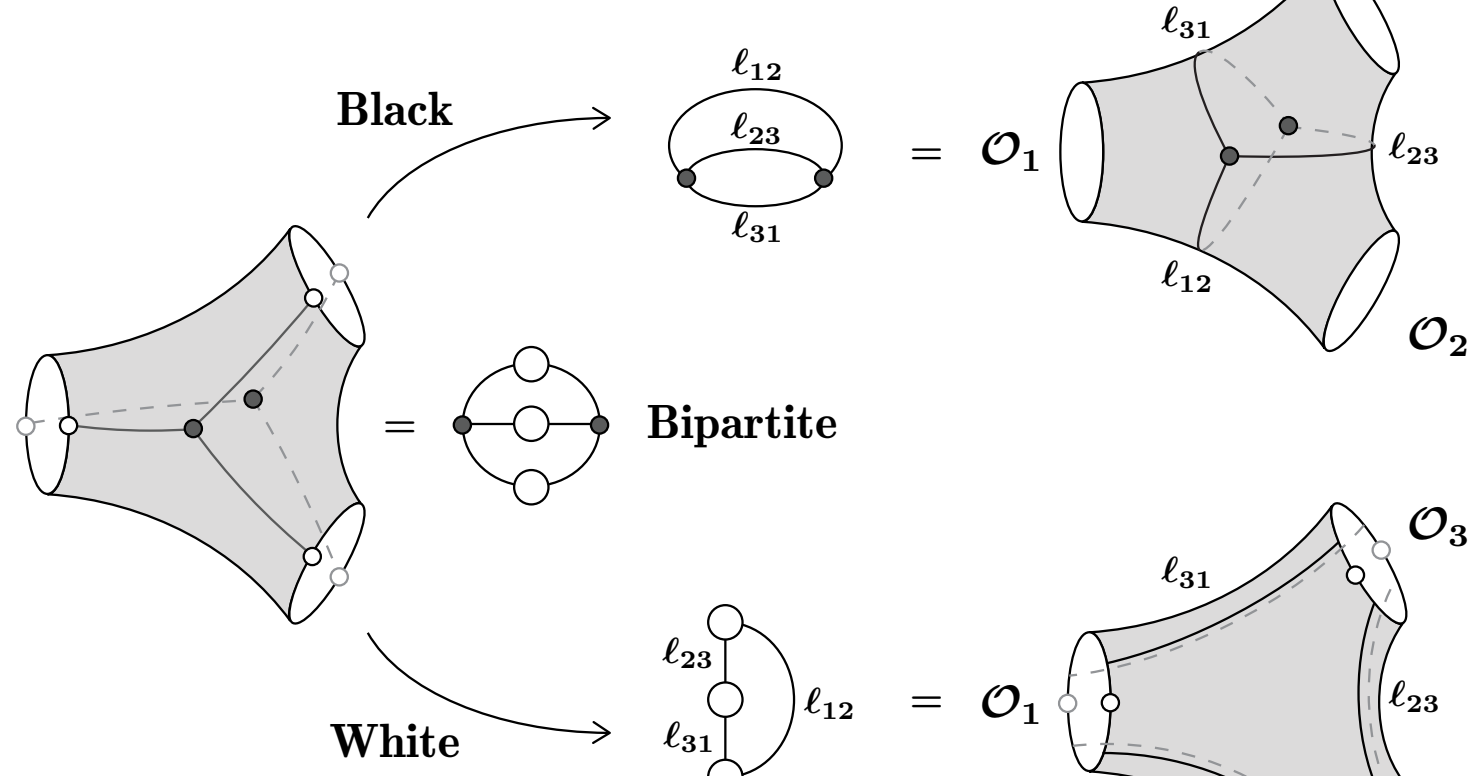

\section{Bipartite}

Figure 29. Three types of the connected ribbon graph with $(g, n)=(0,3)$; bipartite, black and white. The black and white graphs are dual to each other.

This value of $C(\omega)$ remains invariant even if we consider more general $[\omega]$ made by gluing triangles under the constraint (D.6). Hence, the formula (D.21) suggests that higher genus corrections to any $n$-point functions must come in powers of $1 / N_{c}^{2}$, rather than $1 / N_{c}{ }^{40}$

\section{D.6 Counting the dimensions of $\mathcal{M}_{g, n}^{\text {gauge }}$}

We count the dimensions of $\mathcal{M}_{g, n}^{\text {gauge }}$ by identifying its generators as the edges of the ribbon graph.

Suppose that a ribbon graph completely triangulates $\Sigma_{g, n}$. Then, any edge is the boundary of two triangles. Let us make a square by gluing the two triangles, and study the four edges $\left\{\ell_{a}, \ell_{b}, \ell_{c}, \ell_{d}\right\}$ of the square. We define the shift map $\mathfrak{s}_{E}$ on the $E$-th edge as

$$
\mathfrak{s}_{E}:\left\{\ell_{a}, \ell_{b}, \ell_{c}, \ell_{d}\right\} \quad \mapsto\left\{\ell_{a}-1, \ell_{b}+1, \ell_{c}-1, \ell_{d}+1\right\}
$$

which is also shown in figure 30. If the bridge lengths $\left\{\ell_{E}\right\}$ solve the constraint (5.8), the shift map $\mathfrak{s}_{E}$ generates all the other solutions of the constraint.

Denote the $\mathbb{Z}$-module of edge set by

$$
\xi \equiv \bigoplus_{E=1}^{3 n-6+6 g} \xi_{E} \times(E \text {-th edge }), \quad\left(\xi_{E} \in \mathbb{Z}\right)
$$

\footnotetext{
${ }^{40}$ The $1 / N_{c}$ terms appear when the gauge group is $S O$ or $S p$ [79-82]. Also, if the gauge theory has the object in the fundamental representation such as quarks and Wilson loops, the Riemann surface has boundaries [83].
} 

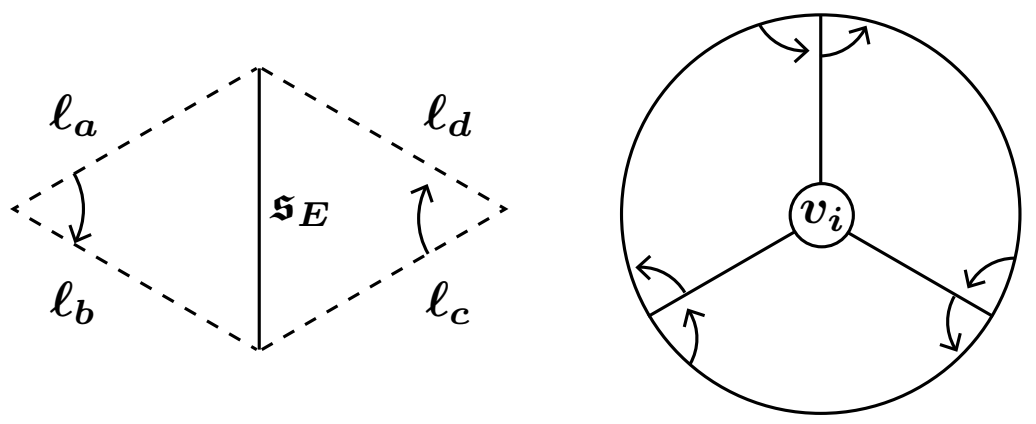

Figure 30. (Left) Shift map $\mathfrak{s}_{E}$ on the $E$-th edge given by (D.22). (Right) Simultaneous shift-maps on all edges connected to $v_{i}$ do not change the bridge lengths $\left\{\ell_{E}\right\}$.

We define the simultaneous shift maps associated to $\xi$, by applying the shift maps $\xi_{E}$ times for the $E$-th edge,

$$
\mathfrak{s}(\xi) \equiv \bigoplus_{E=1}^{3 n-6+6 g} \xi_{E} \mathfrak{s}_{E} .
$$

Let us count the number of the linearly-independent simultaneous shift maps. Define $\Xi_{v_{i}}$ as the set of all edges connected to the $i$-th vertex. The simultaneous shift-map on this set is always trivial,

$$
\mathfrak{s}\left(\Xi_{v_{i}}\right)=\text { identity, } \quad \Xi_{v_{i}}=\bigoplus_{\partial E \ni v_{i}}(E \text {-th edge }), \quad(i=1,2, \ldots n) .
$$

Since there are no more relations among $\mathfrak{s}_{E}$, the number of linearly-independent simultaneous shift maps is equal to $2 n-6+6 g$. This is equal to $\operatorname{dim}_{\mathbb{R}} \mathcal{M}_{g, n}$ in (5.5), which implies (5.17).

\section{E Details of the skeleton reduction}

We explain how to compute the skeleton reduction of $G_{n}$ in order to find $\mathcal{M}_{g, n}^{\text {gauge }}\left(\left\{L_{i}\right\}\right)$. The attached supplementary material Mathematica files implement the procedures given below.

We need a few preliminary steps. To begin with, we fix $\left\{\mathcal{O}_{i}\right\}$ and compute the connected $n$-point functions of single-trace operators in the standard way, e.g. [84]. Let us write the result as a function acting on a set of Feynman graphs $\{\Gamma\}$,

$$
\left(G_{n}\right)_{\text {connected }}=\sum_{\Gamma} F(\Gamma) .
$$

Since $\left(G_{n}\right)_{\text {connected }}$ is long and complicated, we study its cyclic decomposition,

$$
\begin{aligned}
\left(G_{n}\right)_{\text {connected }} & =\sum_{z \in \mathbb{Z}_{\alpha}} z \cdot \mathscr{G}_{n}, \quad \mathscr{G}_{n}=\sum_{\Gamma_{0}} \frac{1}{\left|\operatorname{Aut}_{\mathbb{Z}_{\alpha}}\left(\Gamma_{0}\right)\right|} F\left(\Gamma_{0}\right) \\
\operatorname{Aut}_{\mathbb{Z}_{\alpha}}\left(\Gamma_{0}\right) & =\operatorname{Stab}_{\mathbb{Z}_{\alpha}}\left(\Gamma_{0}\right) \equiv\left\{\boldsymbol{z} \in \mathbb{Z}_{\alpha} \mid \boldsymbol{z} \cdot \Gamma_{0}=\Gamma_{0}\right\} .
\end{aligned}
$$


Here the action $\boldsymbol{z} \cdot \Gamma_{0}$ is given in (4.47). The division by $\left|\mathrm{Aut}_{\mathbb{Z}_{\alpha}} \Gamma_{0}\right|$ is needed to avoid double counting. For each term of $\mathscr{G}_{n}$, we can extract $W \in \mathbb{Z}_{2}^{\otimes L}$ in the notation of section 2.1. Then we change the labeling so that $W$ becomes $W^{\prime}=\prod_{p=1}^{L}\left(p p^{\prime}\right)$. Using the new labeling we compute $\omega=\alpha W$ for each set of Wick-contractions.

In order to apply the skeleton reduction, we need to determine the end-points of open two-point functions. Once we know the open end-points, we can decompose $W$ into $(V, \boldsymbol{\tau})$. At the same time, we obtain $\bar{\alpha}$ after relabeling the skeleton-reduced Wick edges. The set of $\nu=\bar{\alpha} V$ defines the gauge theory moduli space as in (4.58).

There are two methods to determine open end-points and perform our skeleton reduction. The first method is intuitive but slow. The second method needs detailed case-studies, but efficient particularly when $L$ is small.

Choose the canonical ordering of $W=\prod\left(p, p^{\prime}\right)$ and introduce the notation for the Wick-edge, ${ }^{41}$

$$
\mathbf{p}=\left(p, p^{\prime}\right)
$$

We introduce adjacency between Wick-edges, by saying that the Wick-edge $\mathbf{p}$ is adjacent to the following four Wick-edges,

$$
(\alpha(p), W \alpha(p)), \quad\left(\alpha^{-1}(p), W \alpha^{-1}(p)\right), \quad\left(W \alpha\left(p^{\prime}\right), \alpha\left(p^{\prime}\right)\right), \quad\left(W \alpha^{-1}\left(p^{\prime}\right), \alpha^{-1}\left(p^{\prime}\right)\right) .
$$

A pair of edges can be multiply adjacent. Let us call a pair of sequences of consecutive fields between two operators a Wick-edge group. A Wick-edge group of length $\ell$ consists of a pair of $\ell$ consecutive indices which are Wick-contracted within themselves,

$$
\begin{aligned}
\mathcal{E}= & \left\{\left(p, \alpha(p), \alpha^{2}(p), \ldots, \alpha^{\ell-1}(p)\right),\left(q, \alpha(q), \alpha^{2}(q), \ldots, \alpha^{\ell-1}(q)\right) \mid\right. \\
& \text { any } \left.\alpha^{m}(p) \text { for } 0 \leq m<\ell \text { is Wick-contracted with some } \alpha^{n}(q) \text { for } 0 \leq n<\ell\right\} .
\end{aligned}
$$

We also use the notation $\mathcal{E}=\left[\mathbf{p}_{1} \mathbf{p}_{2} \ldots \mathbf{p}_{\ell}\right]$ using edge labels. We look for the maximal Wick-edge group containing $\mathbf{p}$, assuming that $\mathbf{p}$ connects $\mathcal{O}_{i_{p}}$ and $\mathcal{O}_{j_{p}}$. Figure 31 shows examples.

First method. Define the range of adjacency

$$
\begin{aligned}
& \mathcal{R}_{1} \equiv\left\{[-s, t] \mid\left(W \alpha^{n}\left(p^{\prime}\right), \alpha^{n}\left(p^{\prime}\right)\right) \text { connects } \mathcal{O}_{i_{p}} \text { and } \mathcal{O}_{j_{p}} \text { for all }-s \leq n \leq t\right\} \\
& \mathcal{R}_{1}^{\prime} \equiv\left\{\left[-s^{\prime}, t^{\prime}\right] \mid\left(\alpha^{n}(p), W \alpha^{n}(p)\right) \text { connects } \mathcal{O}_{i_{p}} \text { and } \mathcal{O}_{j_{p}} \text { for all }-s^{\prime} \leq n \leq t^{\prime}\right\} .
\end{aligned}
$$

We study the other side of Wick-contractions starting from $n \in \mathcal{R}_{1}$. Since both $p$ and $W \alpha^{n}\left(p^{\prime}\right)$ belong to $\mathcal{O}_{i_{p}}$, we can define an integer $u_{n}$ such that $W \alpha^{n}\left(p^{\prime}\right)=\alpha^{u_{n}}(p)$. Similarly, we can define $W \alpha^{n}(p)=\alpha^{u_{n}^{\prime}}\left(p^{\prime}\right)$ for $n \in \mathcal{R}_{1}^{\prime}$. They are summarized as

$$
\begin{gathered}
\left(W \alpha^{n}\left(p^{\prime}\right), \alpha^{n}\left(p^{\prime}\right)\right) \equiv\left(\alpha^{u_{n}}(p), \alpha^{n}\left(p^{\prime}\right)\right)=\left(\alpha^{u_{n}}(p), W \alpha^{u_{n}}(p)\right), \quad\left(n \in \mathcal{R}_{1}\right) \\
\left(\alpha^{n}(p), W \alpha^{n}(p)\right) \equiv\left(\alpha^{n}(p), \alpha^{u_{n}^{\prime}}\left(p^{\prime}\right)\right)=\left(W \alpha^{u_{n}^{\prime}}\left(p^{\prime}\right), \alpha^{u_{n}^{\prime}}\left(p^{\prime}\right)\right), \quad\left(n \in \mathcal{R}_{1}^{\prime}\right)
\end{gathered}
$$

\footnotetext{
${ }^{41}$ The term Wick-edge is introduced below (2.47).
} 

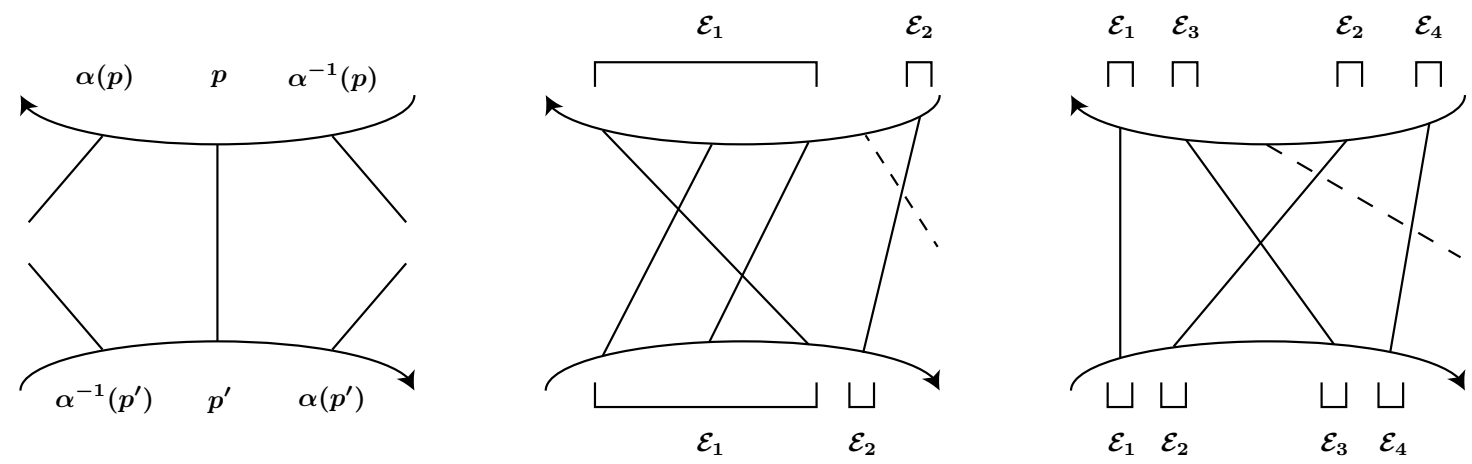

Figure 31. (Left) Four Wick-edges adjacent to the edge $\left(p, p^{\prime}\right)$ given in (E.4). (Center) Two maximal Wick-edge groups $\mathcal{E}_{1}, \mathcal{E}_{2}$. (Right) Four maximal Wick-edge groups $\mathcal{E}_{1} \sim \mathcal{E}_{4}$.

We want to find the ranges $\left(n, n^{\prime}\right) \in\left(\mathcal{R}_{2}, \mathcal{R}_{2}^{\prime}\right)$ such that

$$
\begin{aligned}
& \mathcal{R}_{2} \equiv\left\{\left(\alpha^{m}(p), W \alpha^{m}(p)\right) \text { belongs to } \mathcal{R}_{1} \text { for all } 0 \leq m \leq u_{n} \quad\left(\text { or } u_{n} \leq m \leq 0\right)\right\} \\
& \mathcal{R}_{2}^{\prime} \equiv\left\{\left(W \alpha^{m}\left(p^{\prime}\right), \alpha^{m}\left(p^{\prime}\right)\right) \text { belongs to } \mathcal{R}_{1}^{\prime} \text { for all } 0 \leq m \leq u_{n^{\prime}}^{\prime} \quad\left(\text { or } u_{n^{\prime}}^{\prime} \leq m \leq 0\right)\right\}
\end{aligned}
$$

Clearly $\mathcal{R}_{2} \subset \mathcal{R}_{1}$ and $\mathcal{R}_{2}^{\prime} \subset \mathcal{R}_{1}^{\prime}$. If $\mathcal{R}_{2}, \mathcal{R}_{2}^{\prime}$ are the two maximal Wick-edge groups (E.5), the two sets should be identical.

Conversely said, if either of the conditions (E.6), (E.8) is violated, then we should increase the $\rho$ of $\ell_{i j}^{(\rho)}$ and regard them as different Wick-edges of the skeleton graph.

Second method. Define the edge-adjacency matrix by

$$
J \equiv\left\{J_{\mathbf{p q}}\right\}_{1 \leq \mathbf{p}, \mathbf{q} \leq \bar{L}},
$$$$
J_{\mathbf{p q}}=(\text { Adjacent multiplicity between the Wick-edges } \mathbf{p} \text { and } \mathbf{q}), \quad 0 \leq J_{\mathbf{p q}} \leq 4 \text {. }
$$

If $J_{\mathbf{p q}}=0, \mathbf{p}$ and $\mathbf{q}$ are not adjacent. Some examples of $J_{\mathbf{p q}}$ are shown in figure 32 . Let us denote a pair of operators connected by the edge $\mathbf{p}$ as $\mathcal{O}_{i_{p}}$ and $\mathcal{O}_{j_{p}}$, similarly by the edge q as $\mathcal{O}_{k_{q}}$ and $\mathcal{O}_{l_{q}}$. We have the block decomposition of the matrix $J$ as

$$
J=\sum_{i<j} \sum_{k<l} J^{(i j)(k l)}
$$

We call the elements with $(i j)=(k l)$ diagonal blocks, and the rest off-diagonal blocks. The diagonal part $J^{(i j)(i j)}$ knows how the adjacency inside the Wick-contractions between $\mathcal{O}_{i}$ and $\mathcal{O}_{j}$, somewhat in a cryptic way.

From adjacency relations, we draw the associated adjacency graph. ${ }^{42}$ If $J^{(i j)(i j)}$ is block-diagonal with more than one blocks, then the associated adjacency graph has more than one connected components. Consider the examples. Assuming that the left and right ends of the open two-points are not periodically identified as in figure 33, the following

\footnotetext{
${ }^{42}$ Do not confuse the adjacency graph with the Feynman graphs.
} 

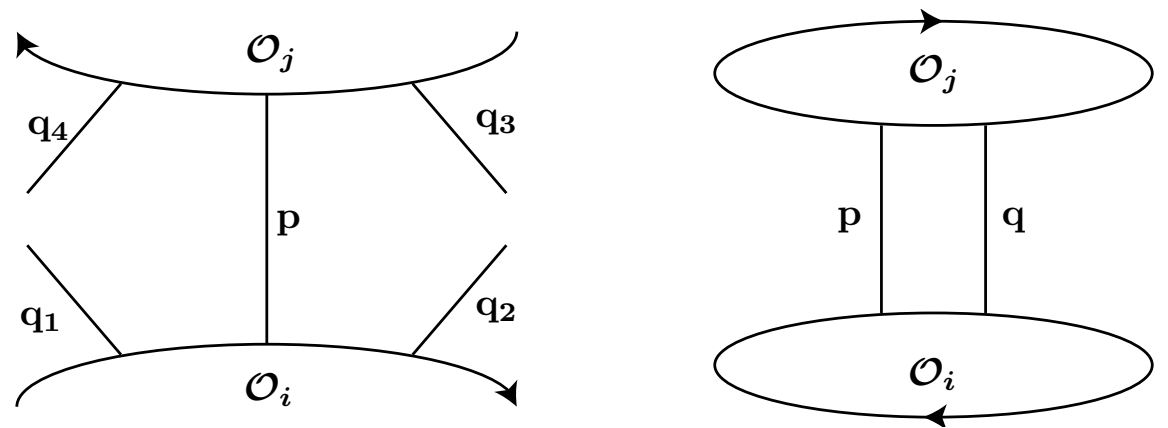

Figure 32. (Left) $J_{\mathbf{p r}}=\sum_{k=1}^{4} \delta\left(\mathbf{r}, \mathbf{q}_{\mathbf{k}}\right)$. Here $\mathbf{p}$ connects $\mathcal{O}_{i}$ and $\mathcal{O}_{j}$. (Right) Example of $J_{\mathbf{p q}}=4$.
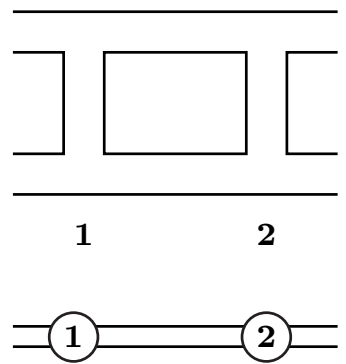
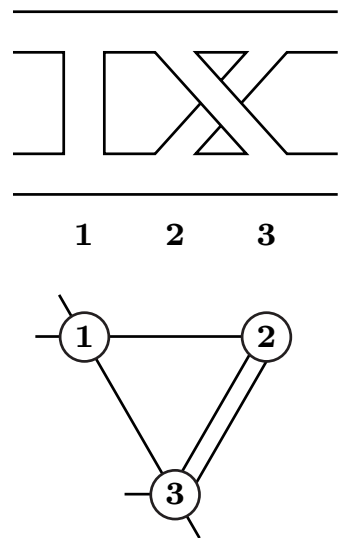
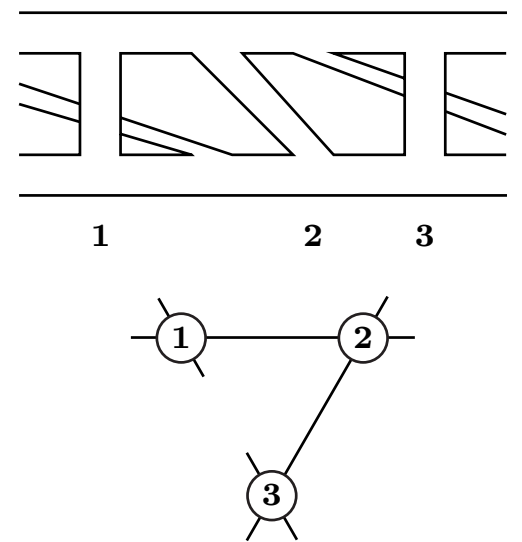

Figure 33. Open two-points (up) and edge-adjacency graphs (down), respectively corresponding to (E.11). In the edge-adjacency graphs, (D) represents a Wick-edge. The edge (or edges) between (D) and (9) means they are (multiply) adjacent.

edge-adjacency matrices corresponds to the Wick-edge groups $\{[12],[123],[1][2][3]\}$,

$$
J^{(i j)(i j)} \sim\left\{\left(\begin{array}{ll}
0 & 2 \\
2 & 0
\end{array}\right), \quad\left(\begin{array}{lll}
0 & 1 & 1 \\
1 & 0 & 2 \\
1 & 2 & 0
\end{array}\right),\left(\begin{array}{lll}
0 & 1 & 0 \\
1 & 0 & 1 \\
0 & 1 & 0
\end{array}\right)\right\} .
$$

In contrast, if we identify the left and right ends of the above operator as in figure 34, then the following edge-adjacency matrices corresponds to the Wick-edge groups $\{[12],[123],[1][2][3]\}$,

$$
J^{(i j)(i j)} \sim\left\{\left(\begin{array}{ll}
0 & 3 \\
3 & 0
\end{array}\right), \quad\left(\begin{array}{lll}
0 & 2 & 1 \\
2 & 0 & 2 \\
1 & 2 & 0
\end{array}\right), \quad\left(\begin{array}{lll}
0 & 1 & 1 \\
1 & 0 & 1 \\
1 & 1 & 0
\end{array}\right)\right\} .
$$

Let us call a length- $\ell$ open two-point periodic if $\ell=\min \left(L_{i}, L_{j}\right)$. In the periodic cases, we need to identify both ends of either $\mathcal{O}_{i}$ or $\mathcal{O}_{j}$ as in figure 34 . 

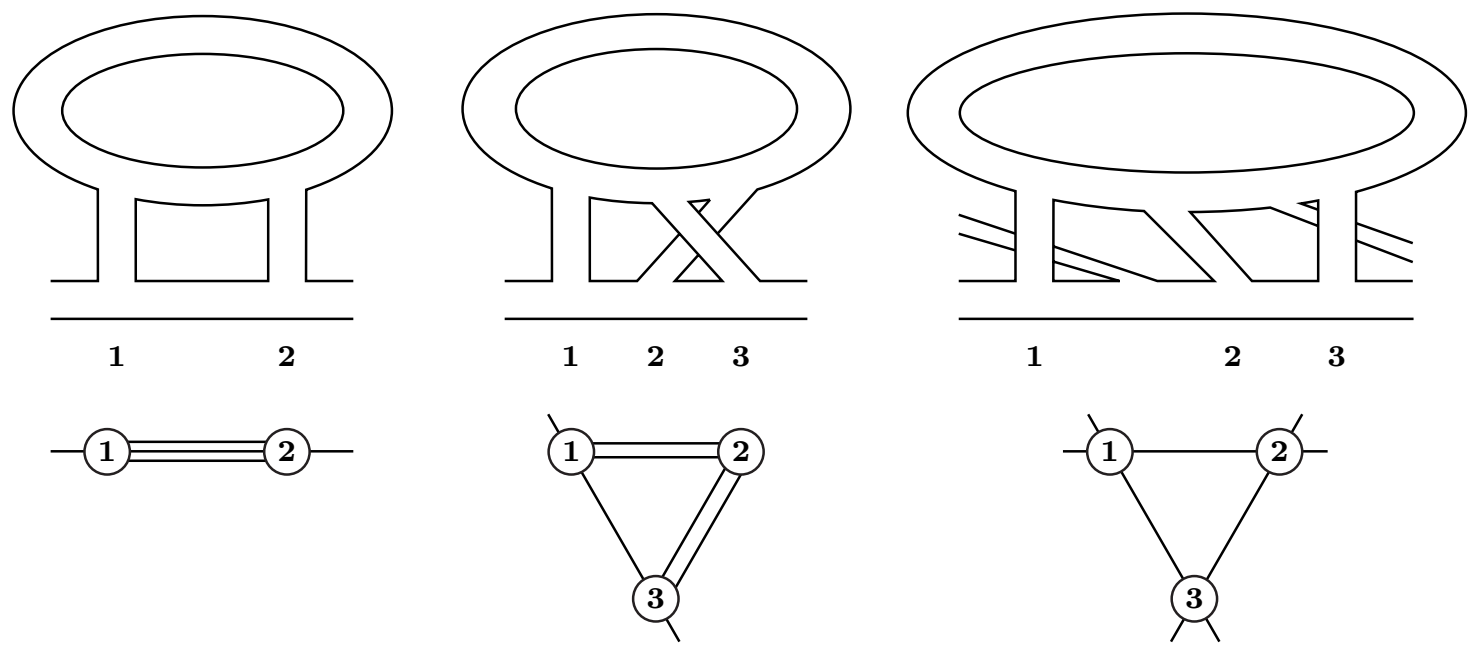

Figure 34. Open two-points and edge-adjacency matrices represented as adjacency graphs for periodic cases.

Notice that two edges go outside the open two-point in the rightmost graphs of figures 33-34. We call them external Wick-edges, because they do not connect $\mathcal{O}_{i}$ and $\mathcal{O}_{j}$ while sitting inside the open two-point between $\mathcal{O}_{i}$ and $\mathcal{O}_{j}$. In non-periodic cases, there are at most four external adjacent Wick-edges. In periodic cases, this number decreases by two (or more).

We can count the number of external Wick-edges adjacent to a given single Wick-edge group, based on $J_{\mathbf{p q}}^{(i j)(i j)}$ and the periodicity. Suppose there is a single Wick-edge group. We divide this group by inserting an external Wick-edge. This reduces the adjacent multiplicity of two edges which belonged to the single Wick-edge group. Therefore, the sum of $J_{\mathbf{p q}}^{(i j)(i j)}$ over all components $\mathbf{p}, \mathbf{q}$ should satisfy

$$
\sum_{\mathbf{p}, \mathbf{q}=1}^{\ell} J_{\mathbf{p q}}^{(i j)(i j)}=4 \ell-4+2(\# \text { of periodic operators })-2(\# \text { of external Wick-edges })
$$

As a corollary, if the adjacency matrix satisfies $\sum_{\mathbf{p}, \mathbf{q}=1}^{\ell} J_{\mathbf{p q}}^{(i j)(i j)} \geq 4 \ell-4+$ 2 (\# of periodic operators), then no external Wick-edges are inserted, and all Wick-edges must belong to the single edge-group $\mathcal{E}$. If this inequality is violated, we need detailed casestudies in order to see how edges are grouped. Some examples are shown in tables $1-2 .{ }^{43}$

\footnotetext{
${ }^{43}$ There are two underlying assumptions in writing this table. First, we consider only those matrices which correspond to a set (or sets) of physically possible Wick-contractions $W$. Second, if several sets of Wick-contractions have the same $J$ and the same periodicity, then they have the same Wick-edge group $\mathcal{E}$. If these assumptions are correct (which seems to be the case), an edge-adjacency matrix $J$ gives the unique corresponding Wick-edge group.
} 


\begin{tabular}{|c|c|c|c|c|c|c|c|}
\hline$J$ & $(0)$ & $\left(\begin{array}{ll}0 & 1 \\
1 & 0\end{array}\right)$ & $\left(\begin{array}{ll}0 & 2 \\
2 & 0\end{array}\right)$ & $\left(\begin{array}{lll}0 & 1 & 0 \\
1 & 0 & 1 \\
0 & 1 & 0\end{array}\right)$ & $\left(\begin{array}{lll}0 & 1 & 1 \\
1 & 0 & 1 \\
1 & 1 & 0\end{array}\right)$ & $\left(\begin{array}{lll}0 & 1 & 0 \\
1 & 0 & 2 \\
0 & 2 & 0\end{array}\right)$ & $\left(\begin{array}{lll}0 & 0 & 1 \\
0 & 0 & 2 \\
1 & 2 & 0\end{array}\right)$ \\
\hline Edge groups & {$[1]$} & {$[1][2]$} & {$[12]$} & {$[1][2][3]$} & {$[1][2][3]$} & {$[1][23]$} & {$[1][23]$} \\
\hline
\end{tabular}

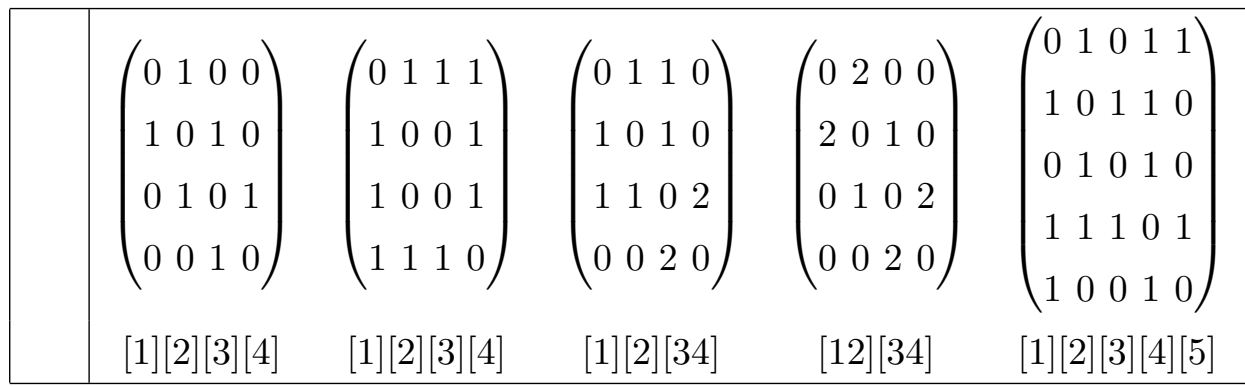

Table 1. Edge-adjacency matrices and the corresponding Wick-edge groups for non-periodic cases.

\begin{tabular}{|c|c|c|c|c|c|c|}
\hline$J$ & $\left(\begin{array}{ll}0 & 2 \\
2 & 0\end{array}\right)$ & $\left(\begin{array}{ll}0 & 3 \\
3 & 0\end{array}\right)$ & $\left(\begin{array}{lll}0 & 1 & 1 \\
1 & 0 & 1 \\
1 & 1 & 0\end{array}\right)$ & $\left(\begin{array}{lll}0 & 1 & 1 \\
1 & 0 & 2 \\
1 & 2 & 0\end{array}\right)$ & $\left(\begin{array}{llll}0 & 2 & 0 & 1 \\
2 & 0 & 1 & 0 \\
0 & 1 & 0 & 2 \\
1 & 0 & 2 & 0\end{array}\right)$ & $\left(\begin{array}{llll}0 & 1 & 1 & 1 \\
1 & 0 & 1 & 1 \\
1 & 1 & 0 & 1 \\
1 & 1 & 1 & 0\end{array}\right)$ \\
\hline Edge groups & {$[1][2]$} & {$[12]$} & {$[1][2][3]$} & {$[1][23]$} & {$[12][34]$} & {$[1][2][3][4]$} \\
\hline
\end{tabular}

Table 2. Edge-adjacency matrices and the corresponding Wick-edge groups for periodic cases.

Open Access. This article is distributed under the terms of the Creative Commons Attribution License (CC-BY 4.0), which permits any use, distribution and reproduction in any medium, provided the original author(s) and source are credited.

\section{References}

[1] G. 't Hooft, A planar diagram theory for strong interactions, Nucl. Phys. B 72 (1974) 461 [INSPIRE].

[2] J.M. Maldacena, The large $N$ limit of superconformal field theories and supergravity, Int. J. Theor. Phys. 38 (1999) 1113 [hep-th/9711200] [INSPIRE].

[3] J.A. Minahan and K. Zarembo, The Bethe ansatz for $N=4$ super Yang-Mills, JHEP 03 (2003) 013 [hep-th/0212208] [inSPIRE].

[4] I. Bena, J. Polchinski and R. Roiban, Hidden symmetries of the $A d S_{5} \times S^{5}$ superstring, Phys. Rev. D 69 (2004) 046002 [hep-th/0305116] [INSPIRE].

[5] N. Beisert, C. Kristjansen and M. Staudacher, The dilatation operator of conformal $N=4$ super Yang-Mills theory, Nucl. Phys. B 664 (2003) 131 [hep-th/0303060] [INSPIRE].

[6] B. Basso, S. Komatsu and P. Vieira, Structure constants and integrable bootstrap in planar $N=4 S Y M$ theory, arXiv:1505.06745 [INSPIRE]. 
[7] B. Eden, Y. Jiang, D. le Plat and A. Sfondrini, Colour-dressed hexagon tessellations for correlation functions and non-planar corrections, JHEP 02 (2018) 170 [arXiv:1710.10212] [INSPIRE].

[8] T. Bargheer, J. Caetano, T. Fleury, S. Komatsu and P. Vieira, Handling handles: nonplanar integrability in $N=4$ supersymmetric Yang-Mills theory, Phys. Rev. Lett. 121 (2018) 231602 [arXiv: 1711.05326] [INSPIRE].

[9] T. Bargheer, J. Caetano, T. Fleury, S. Komatsu and P. Vieira, Handling handles. Part II. Stratification and data analysis, JHEP 11 (2018) 095 [arXiv: 1809.09145] [INSPIRE].

[10] N. Beisert, C. Kristjansen, J. Plefka, G.W. Semenoff and M. Staudacher, BMN correlators and operator mixing in $N=4$ super Yang-Mills theory, Nucl. Phys. B 650 (2003) 125 [hep-th/0208178] [INSPIRE].

[11] S. Corley, A. Jevicki and S. Ramgoolam, Exact correlators of giant gravitons from dual $N=4$ SYM theory, Adv. Theor. Math. Phys. 5 (2002) 809 [hep-th/0111222] [INSPIRE].

[12] T.W. Brown, P.J. Heslop and S. Ramgoolam, Diagonal multi-matrix correlators and BPS operators in $N=4$ SYM, JHEP 02 (2008) 030 [arXiv:0711.0176] [INSPIRE].

[13] R. Bhattacharyya, S. Collins and R. de Mello Koch, Exact multi-matrix correlators, JHEP 03 (2008) 044 [arXiv: 0801.2061] [inSPIRE].

[14] J. Pasukonis and S. Ramgoolam, Quivers as calculators: counting, correlators and Riemann surfaces, JHEP 04 (2013) 094 [arXiv:1301.1980] [INSPIRE].

[15] E. Brézin, C. Itzykson, G. Parisi and J.B. Zuber, Planar diagrams, Commun. Math. Phys. 59 (1978) 35 [INSPIRE].

[16] E. Witten, Two-dimensional gravity and intersection theory on moduli space, Surveys Diff. Geom. 1 (1991) 243 [INSPIRE].

[17] M. Kontsevich, Intersection theory on the moduli space of curves and the matrix Airy function, Commun. Math. Phys. 147 (1992) 1 [INSPIRE].

[18] J. Jenkins, On the existence of certain general extremal metrics, Ann. Math. 66 (1957) 440.

[19] K. Strebel, Quardatic differentials, Springer-Verlag, Berlin Heidelberg, Germany (1984).

[20] J.L. Harer, The virtual cohomological dimension of the mapping class group of an orientable surface, Invent. Math. 84 (1986) 157.

[21] J.L. Harer, The cohomology of the moduli space of curves, Lect. Notes Math. 1337 (1988) 138.

[22] M. Mulase and M. Penkava, Ribbon graphs, quadratic differentials on Riemann surfaces, and algebraic curves defined over $\overline{\mathbb{Q}}$, Asian J. Math. 2 (1998) 875 [math-ph/9811024].

[23] M. Mulase and M. Penkava, Topological recursion for the Poincaré polynomial of the combinatorial moduli space of curves, arXiv:1009.2135 [INSPIRE].

[24] P. Deligne and D. Mumford, The irreducibility of the space of curves of given genus, Publ. Math. I.H.É.S. 36 (1969) 75.

[25] E. D'Hoker and D.H. Phong, The geometry of string perturbation theory, Rev. Mod. Phys. 60 (1988) 917 [INSPIRE].

[26] L. Chekhov, Matrix models and geometry of moduli spaces, hep-th/9509001 [INSPIRE]. 
[27] R. Gopakumar, From free fields to AdS: III, Phys. Rev. D 72 (2005) 066008 [hep-th/0504229] [INSPIRE].

[28] K. Furuuchi, From free fields to AdS: thermal case, Phys. Rev. D 72 (2005) 066009 [hep-th/0505148] [INSPIRE].

[29] O. Aharony, Z. Komargodski and S.S. Razamat, On the worldsheet theories of strings dual to free large $N$ gauge theories, JHEP 05 (2006) 016 [hep-th/0602226] [INSPIRE].

[30] J.R. David and R. Gopakumar, From spacetime to worldsheet: four point correlators, JHEP 01 (2007) 063 [hep-th/0606078] [INSPIRE].

[31] O. Aharony, J.R. David, R. Gopakumar, Z. Komargodski and S.S. Razamat, Comments on worldsheet theories dual to free large $N$ gauge theories, Phys. Rev. D 75 (2007) 106006 [hep-th/0703141] [INSPIRE].

[32] O. Aharony and Z. Komargodski, The space-time operator product expansion in string theory duals of field theories, JHEP 01 (2008) 064 [arXiv:0711.1174] [INSPIRE].

[33] S.S. Razamat, On a worldsheet dual of the Gaussian matrix model, JHEP 07 (2008) 026 [arXiv:0803.2681] [INSPIRE].

[34] S.S. Razamat, From matrices to strings and back, JHEP 03 (2010) 049 [arXiv:0911.0658] [INSPIRE].

[35] S. Charbonnier, B. Eynard and F. David, Large Strebel graphs and $(3,2)$ Liouville CFT, Annales Henri Poincaré 19 (2018) 1611 [arXiv:1709.02709] [INSPIRE].

[36] R. de Mello Koch and S. Ramgoolam, From matrix models and quantum fields to Hurwitz space and the absolute Galois group, arXiv:1002.1634 [INSPIRE].

[37] R. Gopakumar, What is the simplest gauge-string duality?, arXiv:1104.2386 [INSPIRE].

[38] R. Gopakumar and R. Pius, Correlators in the simplest gauge-string duality, JHEP 03 (2013) 175 [arXiv: 1212.1236] [INSPIRE].

[39] R. de Mello Koch and L. Nkumane, Topological string correlators from matrix models, JHEP 03 (2015) 004 [arXiv: 1411.5226] [INSPIRE].

[40] T. Eguchi and S.-K. Yang, The topological $C P^{1}$ model and the large $N$ matrix integral, Mod. Phys. Lett. A 9 (1994) 2893 [hep-th/9407134] [InSPIRE].

[41] C. Kristjansen, J. Plefka, G.W. Semenoff and M. Staudacher, A new double scaling limit of $N=4$ super Yang-Mills theory and PP wave strings, Nucl. Phys. B 643 (2002) 3 [hep-th/0205033] [INSPIRE].

[42] R. Dijkgraaf and E. Witten, Topological gauge theories and group cohomology, Commun. Math. Phys. 129 (1990) 393 [inSPIRE].

[43] M. Fukuma, S. Hosono and H. Kawai, Lattice topological field theory in two-dimensions, Commun. Math. Phys. 161 (1994) 157 [hep-th/9212154] [INSPIRE].

[44] S.-W. Chung, M. Fukuma and A.D. Shapere, Structure of topological lattice field theories in three-dimensions, Int. J. Mod. Phys. A 9 (1994) 1305 [hep-th/9305080] [INSPIRE].

[45] G.W. Moore and G. Segal, D-branes and k-theory in $2 D$ topological field theory, hep-th/0609042 [INSPIRE].

[46] Y. Kimura, S. Ramgoolam and R. Suzuki, Flavour singlets in gauge theory as permutations, JHEP 12 (2016) 142 [arXiv: 1608.03188] [INSPIRE]. 
[47] M. Mulase and M. Penkava, Combinatorial structure of the moduli space of Riemann surfaces and the KP equations, https://www.math.ucdavis.edu/ mulase/texfiles/ 1997moduli.pdf, (1997).

[48] G. Mondello, Riemann surfaces, ribbon graphs and combinatorial classes, in Handbook of Teichmüller theory, volume 2, (2007) [arXiv:0705.1792].

[49] B. Eynard, Counting surfaces, Progr. Math. Phys. 70, Springer, Basel, Switzerland (2016).

[50] Z. Komargodski and A. Zhiboedov, Convexity and liberation at large spin, JHEP 11 (2013) 140 [arXiv: 1212.4103] [INSPIRE].

[51] S.B. Giddings and S.A. Wolpert, A triangulation of moduli space from light cone string theory, Commun. Math. Phys. 109 (1987) 177 [InSPIRE].

[52] S. Nakamura, A calculation of the orbifold Euler number of the moduli space of curves by a new cell decomposition of the Teichmüller space, Tokyo J. Math. 23 (2000) 87.

[53] L. Freidel, D. Garner and S. Ramgoolam, Permutation combinatorics of worldsheet moduli space, Phys. Rev. D 91 (2015) 126001 [arXiv:1412.3979] [InSPIRE].

[54] D. Garner and S. Ramgoolam, The geometry of the light-cone cell decomposition of moduli space, J. Math. Phys. 56 (2015) 112301 [arXiv:1507.02968] [inSPIRE].

[55] L. Hollands and A. Neitzke, Spectral networks and Fenchel-Nielsen coordinates, Lett. Math. Phys. 106 (2016) 811 [arXiv:1312.2979] [INSPIRE].

[56] Y. Kazama and S. Komatsu, On holographic three point functions for GKP strings from integrability, JHEP 01 (2012) 110 [Erratum ibid. 06 (2012) 150] [arXiv:1110.3949] [INSPIRE].

[57] Y. Kazama and S. Komatsu, Wave functions and correlation functions for GKP strings from integrability, JHEP 09 (2012) 022 [arXiv:1205.6060] [INSPIRE].

[58] Y. Kazama and S. Komatsu, Three-point functions in the $\mathrm{SU}(2)$ sector at strong coupling, JHEP 03 (2014) 052 [arXiv: 1312.3727] [InSPIRE].

[59] Y. Kazama, S. Komatsu and T. Nishimura, Classical integrability for three-point functions: cognate structure at weak and strong couplings, JHEP 10 (2016) 042 [Erratum ibid. 02 (2018) 047] [arXiv: 1603. 03164] [INSPIRE].

[60] L.F. Alday and J. Maldacena, Minimal surfaces in AdS and the eight-gluon scattering amplitude at strong coupling, arXiv:0903.4707 [INSPIRE].

[61] L.F. Alday and J. Maldacena, Null polygonal Wilson loops and minimal surfaces in anti-de-Sitter space, JHEP 11 (2009) 082 [arXiv:0904.0663] [INSPIRE].

[62] L.F. Alday, D. Gaiotto and J. Maldacena, Thermodynamic bubble ansatz, JHEP 09 (2011) 032 [arXiv:0911.4708] [INSPIRE].

[63] L.F. Alday, J. Maldacena, A. Sever and P. Vieira, Y-system for scattering amplitudes, J. Phys. A 43 (2010) 485401 [arXiv: 1002.2459] [InSPIRE].

[64] E. Gerchkovitz, J. Gomis, N. Ishtiaque, A. Karasik, Z. Komargodski and S.S. Pufu, Correlation functions of Coulomb branch operators, JHEP 01 (2017) 103 [arXiv: 1602.05971] [INSPIRE].

[65] D. Rodriguez-Gómez and J.G. Russo, Large $N$ correlation functions in superconformal field theories, JHEP 06 (2016) 109 [arXiv: 1604.07416] [INSPIRE]. 
[66] D. Rodriguez-Gomez and J.G. Russo, Operator mixing in large $N$ superconformal field theories on $S^{4}$ and correlators with Wilson loops, JHEP 12 (2016) 120 [arXiv:1607.07878] [INSPIRE].

[67] H. Lin, O. Lunin and J.M. Maldacena, Bubbling AdS space and 1/2 BPS geometries, JHEP 10 (2004) 025 [hep-th/0409174] [INSPIRE].

[68] R. de Mello Koch, J.-H. Huang and L. Tribelhorn, Exciting LLM geometries, JHEP 07 (2018) 146 [arXiv: 1806.06586] [INSPIRE].

[69] A.B. Zamolodchikov and A.B. Zamolodchikov, Factorized $S$ matrices in two-dimensions as the exact solutions of certain relativistic quantum field models, Annals Phys. 120 (1979) 253 [INSPIRE].

[70] L.D. Faddeev, Quantum completely integral models of field theory, Sov. Sci. Rev. C 1 (1980) 107 [INSPIRE].

[71] M. Kim, N. Kiryu, S. Komatsu and T. Nishimura, Structure constants of defect changing operators on the 1/2 BPS Wilson loop, JHEP 12 (2017) 055 [arXiv:1710.07325] [INSPIRE].

[72] A. Cavaglià, N. Gromov and F. Levkovich-Maslyuk, Quantum spectral curve and structure constants in $N=4$ SYM: cusps in the ladder limit, JHEP 10 (2018) 060 [arXiv: 1802.04237] [INSPIRE].

[73] B. Frab and D. Margalit, A primer on mapping class groups, Princeton University Press, Princeton, NJ, U.S.A. (2012).

[74] B. Eynard, Lectures notes on compact Riemann surfaces, arXiv:1805.06405.

[75] B. Zwiebach, How covariant closed string theory solves a minimal area problem, Commun. Math. Phys. 136 (1991) 83 [InSPIRE].

[76] R.C. Penner, Perturbative series and the moduli space of Riemann surfaces, J. Diff. Geom. 27 (1988) 35 [inSPIRE].

[77] V.V. Fock, Description of moduli space of projective structures via fat graphs, hep-th/9312193 [INSPIRE].

[78] S.K. Ashok, F. Cachazo and E. Dell'Aquila, Strebel differentials with integral lengths and Argyres-Douglas singularities, hep-th/0610080 [INSPIRE].

[79] P. Caputa, C. Kristjansen and K. Zoubos, On the spectral problem of $N=4$ SYM with orthogonal or symplectic gauge group, JHEP 10 (2010) 082 [arXiv: 1005.2611] [INSPIRE].

[80] P. Caputa, R. de Mello Koch and P. Diaz, Operators, correlators and free fermions for $\mathrm{SO}(N)$ and $\mathrm{Sp}(N)$, JHEP 06 (2013) 018 [arXiv: 1303.7252] [INSPIRE].

[81] G. Kemp, Restricted Schurs and correlators for $\mathrm{SO}(N)$ and $\mathrm{Sp}(N)$, JHEP 08 (2014) 137 [arXiv: 1406.3854] [INSPIRE].

[82] C. Lewis-Brown and S. Ramgoolam, BPS operators in $N=4 \mathrm{SO}(N)$ super Yang-Mills theory: plethysms, dominoes and words, JHEP 11 (2018) 035 [arXiv:1804.11090] [INSPIRE].

[83] J.M. Maldacena, Wilson loops in large N field theories, Phys. Rev. Lett. 80 (1998) 4859 [hep-th/9803002] [INSPIRE].

[84] P. Vieira, Gamma matrices and Wick contractions, in Mathematica summer school on theoretical physics, (2013). 